\title{
EXTERNALLY DEFINABLE QUOTIENTS AND NIP EXPANSIONS OF THE REAL ORDERED ADDITIVE GROUP
}

\author{
ERIK WALSBERG
}

\begin{abstract}
Let $\mathcal{R}$ be an NIP expansion of $(\mathbb{R},<,+)$ by closed subsets of $\mathbb{R}^{n}$ and continuous functions $f: \mathbb{R}^{m} \rightarrow \mathbb{R}^{n}$. Then $\mathcal{R}$ is generically locally ominimal. It follows that if $X \subseteq \mathbb{R}^{n}$ is definable in $\mathcal{R}$ then the $C^{k}$-points of $X$ are dense in $X$ for any $k \geq 0$. This follows from a more general theorem on NIP expansions of locally compact groups, which itself follows from a result on quotients of definable sets by equivalence relations which are externally definable and $\Lambda$-definable. We also show that $\mathcal{R}$ is strongly dependent if and only if $\mathcal{R}$ is either o-minimal or $(\mathbb{R},<,+, \alpha \mathbb{Z})$-minimal for some $\alpha>0$.
\end{abstract}

A highly saturated structure $\mathcal{M}$ is NIP if whenever $(I,<)$ is an indiscernible sequence in $\mathcal{M}$ and $X$ is an $\mathcal{M}$-definable (possibly with parameters) set, then $X \cap I$ is a finite union of <-convex sets. So we might view NIP structures as "weakly ominimal on indiscernible sequences". We therefore hope that definable sets in NIP expansions of $(\mathbb{R},<,+)$ behave similarly to definable sets in o-minimal expansions of $(\mathbb{R},<,+)$. One result in this direction is the theorem of Simon [74, Corollary 3.7] that a dp-rank one expansion of $(\mathbb{R},<,+)$ is o-minimal. In this paper we show that NIP expansions of $(\mathbb{R},<,+)$ by closed sets are very similar to o-minimal expansions.

Throughout all structures are first order. When we say that something is definable in a structure we mean that is definable possibly with parameters from that structure. Two structures on a common domain $M$ are interdefinable if they define the same subsets of all $M^{n}$. We regard interdefinable structures as the same. Let $\mathcal{M}$ be a structure with domain $M$. The structure induced on $A \subseteq M^{m}$ by $\mathcal{M}$ is the structure with domain $A$ whose primitive $n$-ary relations are all sets of the form $X \cap A^{n}$ for $\mathcal{M}$-definable $X \subseteq M^{m n}$. The structure induced on $A$ eliminates quantifiers if every subset of $A^{n}$ definable in the induced structure is of the form $Y \cap A^{n}$ for $\mathcal{M}$-definable $Y \subseteq M^{m n}$. (We will commonly encounter this situation.)

Suppose $M^{n}$ is equipped with a topology for all $n \geq 1$. In this paper we will always equip $M^{n}$ with the product topology when $n \geq 2$, but the basic definitions are naturally formulated in the more general context. We say that $\mathcal{M}$ is noiseless if every definable subset of every $M^{n}$ either has interior or is nowhere dense. We say that $\mathcal{M}$ is noiseless in one variable if every definable subset of $M$ either has interior or is nowhere dense. We say that $\mathcal{M}$ is strongly noiseless if the induced structure on any definable $Y \subseteq M^{n}$ is noiseless. Equivalently $\mathcal{M}$ is strongly noiseless if whenever $X, Y$ are definable subsets of $M^{n}$ then $X$ is either nowhere dense in $Y$ or has interior in $Y$. We say that $\mathcal{N}$ is strongly noiseless in one variable if whenever $X, Y \subseteq M$ are definable then $X$ is either nowhere dense in $Y$ or has

Date: March 30, 2020. 
interior in $Y$. (These definitions are essentially due to Chris Miller.)

Strong noiselessness is a typical and important property of structures whose definable sets are "tame topological" objects. Algebraically closed fields, (weakly) ominimal expansions of dense linear orders [57, P-minimal expansions of $p$-adically closed fields (in particular the field of $p$-adic numbers) [12, C-minimal expansions of dense C-relations (in particular algebraically closed valued fields) 36, and unstable dp-minimal expansions of fields [49, 78, are all strongly noiseless with respect to canonical topologies. There are many model-theoretically tame structures which are noisey with respect to a canonical topology. In particular there are many noisey NIP expansions of $(\mathbb{R},<,+)$ such as $(\mathbb{R},<,+, \mathbb{Q}),\left(\mathbb{R},<,+, \times, \mathbb{R}_{\text {alg }}\right)$ where $\mathbb{R}_{\text {alg }}$ is the set of real algebraic numbers, and $(\mathbb{R},<,+, \times, U)$ where $U$ is the set of complex roots of unity 34 .

The open core $\mathcal{M}^{\circ}$ of $\mathcal{M}$ is the structure on $M$ whose primitive $n$-ary relations are all closures of $\mathcal{M}$-definable subsets of $M^{n}$. If $\mathcal{M}$ defines a basis for the topology on each $M^{n}$ then $\mathcal{N}^{\circ}$ is the reduct of $\mathcal{M}$ generated by all closed $\mathcal{M}$-definable sets. If the topology on $M^{n}$ is Hausdorff, $X \subseteq M^{m}$ is $\mathcal{M}$-definable and either open or closed, and $f: X \rightarrow M^{n}$ is continuous and $\mathcal{M}$-definable, then $f$ is definable in $\mathcal{M}^{\circ}$. (Hausdorffness ensures that the graph of $f$ is closed in $X \times M^{n}$.) We say $\mathcal{M}$ is generated by closed sets or is an expansion by closed sets if it is interdefinable with $\mathcal{M}^{\circ}$. A subset of a topological space is constructible if it is a boolean combination of closed sets. The following result of Miller and Dougherty [19] shows that if $\mathcal{M}$ defines a basis for the topology on each $M^{n}$ then $\mathcal{M}^{\circ}$ is interdefinable with the reduct of $\mathcal{M}$ generated by all constructible $\mathcal{M}$-definable sets.

Fact 0.1. Suppose $X$ is an $\mathcal{M}$-definable set, $\mathcal{M}$ defines a basis for a topology on $X$, and $Y \subseteq X$ is constructible and definable. Then $Y$ is a boolean combination of definable closed sets.

It has been observed that $\mathcal{M}^{\circ}$ is typically noiseless when $\mathcal{M}$ is model-theoretically tame and $\mathcal{M}$ is typically interdefinable with $\mathcal{M}^{\circ}$ when $\mathcal{M}$ is strongly noiseless (the latter usually follows from some kind of cell decomposition). It is conjectured that $\mathcal{R}^{\circ}$ is noiseless if $\mathcal{R}$ expands $(\mathbb{R},<,+, \times)$ and does not define the set of integers. It is also conjectured that $\mathcal{R}^{\circ}$ is strongly noiseless when $\mathcal{R}$ expands $(\mathbb{R},<,+)$ and does not interpret the monadic second order theory of one successor. (We discuss these conjectures in Section 2.2) Theorem A generalizes a special case.

Theorem A. Suppose $G$ is a group and $\mathcal{G}$ is a first order expansion of $G$ such that $\mathcal{G}$ defines

(1) a basis for a locally compact Hausdorff group topology on $G$,

(2) a family $\mathcal{K}$ of compact subsets of $G$ such that any compact subset of $G$ is contained in some element of $\mathcal{K}$.

If $\mathcal{G}$ is NIP then $\mathcal{G}^{\circ}$ is strongly noiseless.

Note that (2) is superfluous when $G$ is compact. We do not know how strong the conclusion of Theorem $\mathrm{A}$ is in general. It implies that $\mathcal{G}^{\circ}$-definable functions are generically continuous, see Proposition 7.6. We will see that Theorem A has very strong consequences over $(\mathbb{R},<,+)$. We first describe the proof of Theorem $\mathrm{A}$ and then discuss its consequences over $(\mathbb{R},<,+)$. Theorem $\mathrm{A}$ is a consequence of Theorem B, which we believe to be of independent interest. 
Theorem B. Suppose $\mathcal{N}$ is a highly saturated NIP structure and $X$ is an $\mathcal{N}$ definable set. Let $E$ be an equivalence relation on $X$ which is both $\Lambda$-definable and externally definable. Suppose that the Shelah expansion $\mathcal{N}^{\text {Sh }}$ of $\mathcal{N}$ defines a basis for the logic uniformity on $X / E$. Then the structure induced on $X / E$ by $\mathcal{N}^{\text {Sh }}$ is strongly noiseless.

Suppose $\mathcal{N}$ is a highly saturated o-minimal expansion of field and $G$ is a definably compact group. Then $G^{00}$ is known to be externally definable and the structure induced on $G / G^{00}$ by $\mathcal{N}^{\mathrm{Sh}}$ is interpretable in a disjoint union of finitely many o-minimal structures. In particular the induced structure on $G / G^{00}$ is strongly noiseless. We view Theorem B as a broad generalization of this fact. As an application of Theorem $\mathrm{B}$ we show that if $\mathcal{N}$ is a highly saturated strongly dependent structure, $G$ is a definably amenable group, and $G / G^{00}$ is a simple centerless Lie group, then the structure induced on $G / G^{00}$ by $\mathcal{N}^{\mathrm{Sh}}$ is bi-interpretable with an o-minimal expansion of the real field. We prove this theorem and discuss an interesting possible generalization in Section 14 below.

We now describe the proof of Theorem A from Theorem B. Let $\mathcal{N}$ be a highly saturated elementary extension of $\mathcal{G}$. We let Fin be the subgroup of "finite" elements of $\mathcal{N}$ and Inf be the subgroup of "infinitesimal" elements of $\mathcal{N}$. We show that Inf is $\bigwedge$-definable and Fin, Inf are both externally definable. Using familiar ideas from nonstandard analysis we see that Fin/Inf can be identified with $G$ and the quotient map Fin $\rightarrow$ Fin/Inf can be identified with the usual standard part map. We also see that the logic topology agrees with the group topology on $G$. Finally, we show that $\mathcal{G}^{\circ}$ is a reduct of the structure induced on $G$ by $\mathcal{N}^{\text {Sh }}$. Our proof does not rely on the group structure on $G$, only on the induced uniform structure. For this reason our proof goes through in the more general setting of locally compact Hausdorff uniform spaces, see Section 7 . The general result is reasonably sharp, see Section 8

We now describe consequences of Theorem A over the reals. Suppose $\mathcal{R}$ is an expansion of $(\mathbb{R},<)$. We say that $\mathcal{R}$ is locally o-minimal if for every $\mathcal{R}$-definable $X \subseteq \mathbb{R}$ and $a \in X$ there is an open interval $I$ containing $a$ such that $I \cap X$ is definable in $(\mathbb{R},<)$. By [80, Corollary 3.4] $\mathcal{R}$ is locally o-minimal if and only if the induced structure on any bounded interval is o-minimal. We say that $\mathcal{R}$ is generically locally o-minimal if for every $\mathcal{R}$-definable $X \subseteq \mathbb{R}$ there is a dense definable open $V \subseteq X$ such that for all $a \in V$ there is an open interval $I$ containing $a$ such that $I \cap X$ is definable in $(\mathbb{R},<)$. It is known that an expansion of $(\mathbb{R},<,+)$ is strongly noiseless if and only if it is generically locally o-minimal (see Theorem 2.17 below). So Theorem C follows from Theorem B.

Theorem C. Suppose $\mathcal{R}$ is an expansion of $(\mathbb{R},<,+)$. If $\mathcal{R}$ is NIP then $\mathcal{R}^{\circ}$ is generically locally o-minimal.

Theorem $\mathrm{C}$ fails over $(\mathbb{R},<)$. Section 8 shows that if $f:[0,1] \rightarrow[0,1]$ is the classical Cantor function, aka "devil's staircase", then $(\mathbb{R},<, f)$ is dp-minimal and noisey.

We say that $\mathcal{N}$ is $\mathcal{M}$-minimal if $\mathcal{N}$ is an expansion of $\mathcal{M}$ and every $\mathcal{N}$-definable subset of $M$ is $\mathcal{M}$-definable. (If $\mathcal{M}=(\mathbb{R},<)$ then $\mathcal{M}$-minimality is o-minimality.) In Section 11 we prove Theorem D by combining Theorem C, results of Dolich and 
Goodrick [17] on strongly dependent expansions of ordered abelian groups, work of Kawakami, Takeuchi, Tanaka, and Tsuboi [51] on locally o-minimal structures, and a recent result of Bès and Choffrut on $(\mathbb{R},<,+, \mathbb{Z})[6]$.

Theorem D. Suppose $\mathcal{R}$ is an expansion of $(\mathbb{R},<,+)$. The following are equivalent.

(1) $\mathcal{R}$ is a strongly dependent expansion by closed sets.

(2) $\mathcal{R}$ is strongly dependent and noiseless.

(3) $\mathcal{R}$ is either o-minimal or $(\mathbb{R},<,+, \alpha \mathbb{Z})$-minimal for some $\alpha>0$.

(4) $\mathcal{R}$ is either o-minimal or interdefinable with $(\mathbb{R},<,+, \mathcal{B}, \alpha \mathbb{Z})$ for some collection $\mathcal{B}$ of bounded sets such that $(\mathbb{R},<,+, \mathcal{B})$ is o-minimal.

(5) $\mathcal{R}$ is either o-minimal or locally o-minimal and interdefinable with $(\mathcal{S}, \alpha \mathbb{Z})$ for some o-minimal expansion $\mathcal{S}$ of $(\mathbb{R},<,+)$.

In each case above $\alpha$ is unique up to rational multiples. Suppose $\mathcal{R}$ is $(\mathbb{R},<,+, \alpha \mathbb{Z})$ minimal. Then every definable subset of $\mathbb{R}^{n}$ is a finite union of sets of the form $\bigcup_{b \in B} b+A$ for $(\alpha \mathbb{Z},<,+)$-definable $B \subseteq(\alpha \mathbb{Z})^{n}$ and $\mathcal{R}$-definable $A \subseteq[0, \alpha)^{n}$. It follows that $\mathcal{R}$ is bi-interpretable with the disjoint union of the induced structure on $[0, \alpha)$ (which is o-minimal) and $(\mathbb{Z},<,+)$.

Informally, Theorem D shows that a strongly dependent expansion by closed sets which is not o-minimal has a canonical decomposition into an "integer part", $(\alpha \mathbb{Z},<,+)$, and an o-minimal "fractional part", the induced structure on $[0, \alpha)$.

Theorem $\mathrm{D}$ shows that a strongly dependent expansions of $(\mathbb{R},<,+)$ by closed sets is almost o-minimal. In Section 10 we apply the general theory of expansions of $(\mathbb{R},<,+)$ to show that generically locally o-minimal expansions of $(\mathbb{R},<,+)$ enjoy many of the good properties of o-minimal structures. Generic local o-minimality implies definable selection, generic $C^{k}$-smoothness of definable functions, and yields a theory of dimension for definable sets well suited to computations. We use these tools to obtain a dichotomy between "linear" and "field-type" expansions.

Theorem E. Suppose $\mathcal{R}$ is a generically locally o-minimal expansion of $(\mathbb{R},<,+)$. Then the following are equivalent:

(1) there is a nonempty open interval $I$ and continuous definable $\oplus, \otimes: I^{2} \rightarrow I$ such that $(I,<, \oplus, \otimes)$ is an ordered field isomorphic to $(\mathbb{R},<,+, \times)$,

(2) there is a definable field $(Z, \oplus, \otimes)$ such that $\operatorname{dim} Z \geq 1$,

(3) there is a definable family $\left(X_{a}\right)_{a \in B}$ of one-dimensional subsets of $\mathbb{R}^{m}$ such that $\operatorname{dim} B \geq 2$ and $X_{a} \cap X_{b}$ is zero-dimensional for distinct $a, b \in B$,

(4) there is a definable function $f: U \rightarrow \mathbb{R}^{n}, U$ a definable open subset of $\mathbb{R}^{m}$, such that $f$ is not locally affine on a dense open subset of $U$,

(5) there is a definable subset $X$ of $\mathbb{R}^{m}$ such that the set of $p \in X$ such that $U \cap X=H \cap X$ for some open neighbourhood $U$ of $p$ and affine subspace $H$ of $\mathbb{R}^{m}$ is not dense in $X$.

There are locally o-minimal expansions of $(\mathbb{R},<,+)$ which define infinite fields and do not satisfy (1) above, see Fact 2.1. We conjecture that an NIP expansion of $(\mathbb{R},<,+)$ by closed sets which defines an infinite field satisfies (1), see Conjecture 4. We prove several special cases of this conjecture, in particular the strongly dependent case.

The straightforward analogues of Theorems C and D fail for archimedean structures. In Section 13.2 we treat the correct analogue. Suppose $\mathcal{R}$ expands a dense 
archimedean ordered abelian group $(R,<,+)$, which we take to be a substructure of $(\mathbb{R},<,+)$. Laskowski and Steinhorn [54] show that if $\mathcal{R}$ is o-minimal then $\mathcal{R}$ is an elementary substructure of a unique o-minimal expansion $\mathcal{S}$ of $(\mathbb{R},<,+)$. In this case the structure induced on $R$ by $\mathcal{S}$ is interdefinable with the Shelah expansion $\mathcal{R}^{\mathrm{Sh}}$ of $\mathcal{R}$. It follows from work of Wencel $[90$, that if $\mathcal{R}$ is weakly o-minimal then there is an o-minimal expansion $\mathcal{R}^{\square}$ of $(\mathbb{R},<,+)$ such that the structure induced on $R$ by $\mathcal{R}^{\square}$ is interdefinable with $\mathcal{R}^{\mathrm{Sh}}$. Theorem $\mathrm{F}$ generalizes these results.

Theorem F. Let $R$ be a dense subgroup of $(\mathbb{R},+), \mathcal{R}$ be an NIP expansion of $(R,<,+)$, and $\mathcal{R} \prec \mathcal{N}$ be highly saturated. Let $\mathbf{F i n}$ be the convex hull of $R$ in $N$ and Inf be the set of $a \in N$ such that $|a|<b$ for all $b \in R, b>0$. Identify $\mathbf{F i n} / \mathbf{I n f}$ with $\mathbb{R}$ and let st : Fin $\rightarrow \mathbb{R}$ be the quotient map. As Fin and Inf are $\mathcal{N}^{\mathrm{Sh}}$ definable we regard $\mathbb{R}$ as an imaginary sort of $\mathcal{N}^{\mathrm{Sh}}$. Then the following structures are interdefinable.

(1) The structure $\mathcal{R}^{\square}$ on $\mathbb{R}$ with an $n$-ary relation symbol defining the closure in $\mathbb{R}^{n}$ of every subset of $R^{n}$ which is externally definable in $\mathcal{R}$.

(2) The structure on $\mathbb{R}$ with an $n$-ary relation defining $\operatorname{st}\left(\mathbf{F i n}^{n} \cap X\right)$ for every $\mathcal{N}$-definable $X \subseteq N^{n}$.

(3) The open core of the structure induced on $\mathbb{R}$ by $\mathcal{N}^{\mathrm{Sh}}$.

Furthermore $\mathcal{R}^{\square}$ is generically locally o-minimal and if $\mathcal{R}$ is strongly dependent then $\mathcal{R}^{\square}$ is either o-minimal or $(\mathbb{R},<,+, \alpha \mathbb{Z})$-minimal for some $\alpha>0$. The structure induced on $R$ by $\mathcal{R}^{\square}$ is a reduct of $\mathcal{R}^{\mathrm{Sh}}$ and if $\mathcal{R}$ is strongly dependent and noiseless then the structure induced on $R$ by $\mathcal{R}^{\square}$ eliminates quantifiers and is interdefinable with $\mathcal{R}^{\mathrm{Sh}}$.

The final two sections are somewhat speculative. In Section 14 we discuss some questions arising out of work on definable groups in o-minimal structures. In Section 15 we discuss how our results could relate to a possible notion of modularity or one-basedness for NIP structures. (It is a known open problem to define a good notion of modularity for NIP structures.) We also give an example, perhaps suprising, of an NIP structure $\mathcal{N}$ such that $\mathcal{M}$ does not interpret an infinite field but the Shelah expansion $\mathcal{M}^{\mathrm{Sh}}$ of $\mathcal{M}$ interprets $(\mathbb{R},<,+, \times)$.

\section{NOTATION AND CONVENTIONS}

Throughout $m, n, k, d$ are natural numbers, $i, j$ are integers, and $s, t, \lambda, \alpha$ are real numbers. We let $\mathbb{R}_{>0}$ be the set of positive real numbers. We consider $\mathbb{R}^{0}$ to be a singleton. Given a subset $X$ of $A \times B$ and $a \in A$ we let $X_{a}$ be $\{b \in B:(a, b) \in X\}$. We let $\operatorname{gr}(f) \subseteq A \times B$ be the graph of a function $f: A \rightarrow B$.

We say that a family of $\mathcal{C}$ of sets is subdefinable if it is a subfamily of a definable family of sets. A subdefinable basis for a topology on a definable set $X$ is a subdefinable family of sets forming a basis for a topology on $X$.

We let $\mathbb{R}_{\text {vec }}$ be the ordered vector space $\left(\mathbb{R},<,+,(t \mapsto \lambda t)_{\lambda \in \mathbb{R}}\right)$ of real numbers.

We let $\mathrm{Cl}(X)$ be the closure and $\operatorname{Bd}(X)$ be the boundary of a subset $X$ of a topological space. 
A Cantor subset of $\mathbb{R}$ is a nowhere dense compact subset of $\mathbb{R}$ without isolated points, equivalently, a subset of $\mathbb{R}$ which is homeomorphic to the classical middle thirds Cantor set.

We let $\operatorname{dim} X$ be the topological dimension of a subset $X$ of $\mathbb{R}^{n}$ and "dimension" without modification always means "topological dimension". There are several notions of topological dimension which need not agree on general topological spaces but do agree on separable metric spaces. So in particular there is a canonical notion of topological dimension for subsets of Euclidean space. We refer to Engelking [24] for definitions and results on topological dimension. Recall that $X \subseteq \mathbb{R}^{n}$ is zerodimensional if and only if $X$ is nonempty and totally disconnected. By convention $\operatorname{dim} X=-1$ if and only if $X$ is empty.

We will sometimes work in a multi-sorted setting. Suppose $L$ is a language with $S$ the set of sorts and $\mathcal{M}$ is an $L$-structure. Then we let $M$ denote the $S$-indexed family $\left(M_{s}\right)_{s \in S}$ of underlying sets of the sorts of $\mathcal{M}$. If $x=\left(x_{j}\right)_{j \in J}$ is a tuple of variables, we let $M^{x}=\prod_{j \in J} M_{s\left(x_{j}\right)}$ where $M_{s\left(x_{j}\right)}$ is the sort of the variable $x_{j}$. If $\varphi(x, y)$ is an $L$-formula and $b \in M^{y}$, we let $\varphi\left(M^{y}, b\right)$ be the set defined by $\varphi(x, b)$.

Suppose $\mathcal{N}$ is a highly saturated structure. A subset $X$ of $N^{x}$ is $\bigwedge$-definable if it is the intersection of a small collection of $\mathcal{N}$-definable sets. Such sets are often said to be "type-definable".

\section{BACKGround on Expansions of $(\mathbb{R},<,+)$}

Throughout this section $\mathcal{R}$ is an expansion of $(\mathbb{R},<,+)$ and "definable" without modification means "R-definable". In Section 2.1 we describe some important examples of expansions of $(\mathbb{R},<,+)$. In Section 2.2 we discuss the core conjectures on expansions of $(\mathbb{R},<,+)$. In Section 2.3 we give various equivalent definitions of local and generic local o-minimality. In particular we show that an expansion of $(\mathbb{R},<,+)$ is generically locally o-minimal if and only if it is strongly noiseless. These results are well-known to experts. In Section 2.4 we give some background on d-minimal expansions. These results are already essentially known to experts, but our approach is new. In particular we introduce the "Pillay rank".

The general study of expansions of $(\mathbb{R},<,+)$ was envisioned by Chris Miller.

2.1. Examples of expansions. The basic example of an expansion which is locally o-minimal and not o-minimal is $(\mathbb{R},<,+, \mathbb{Z})$. (Miller 62 and Weispfenning [89] independently showed that $(\mathbb{R},<,+, \mathbb{Z})$ has quantifier elimination in a natural expanded language, local o-minimality follows.) Marker and Steinhorn showed in unpublished work that $(\mathbb{R},<,+, \sin )$ is locally o-minimal, see [80. Fact 2.1 generalizes both of these examples. Fact 2.1 is due to Kawakami, Takeuchi, Tanaka, and Tsuboi, it is a special case of [51, Theorem 18]. Given a real number $\alpha>0$ we let $+_{\alpha}:[0, \alpha)^{2} \rightarrow[0, \alpha)$ be given by $t+{ }_{\alpha} t^{\prime}=t+t^{\prime}$ when $t+t^{\prime}<\alpha$ and $t+\alpha t^{\prime}=t+t^{\prime}-\alpha$ otherwise.

Fact 2.1. Fix $\alpha>0$. Suppose $\mathcal{J}$ is an o-minimal expansion of $\left([0, \alpha),<,+_{\alpha}\right)$ and $\mathcal{D}$ is an arbitrary first order expansion of $(\alpha \mathbb{Z},<,+)$. Then there is a first order expansion $\mathcal{S}$ of $(\mathbb{R},<,+)$ such that a subset of $\mathbb{R}^{n}$ is $\mathcal{S}$-definable if and only if it is 
a finite union of sets of the form

$$
\bigcup_{b \in B} b+A
$$

for J-definable $A \subseteq[0, \alpha)^{n}$ and $\mathcal{D}$-definable $B \subseteq(\alpha \mathbb{Z})^{n}$. This $\mathcal{S}$ is locally o-minimal and is bi-interpretable with the disjoint union of $\mathcal{J}$ and $\mathcal{D}$.

If $\alpha=1, \mathcal{J}$ is $\left([0,1),<,+{ }_{1}\right)$, and $\mathcal{D}$ is $(\mathbb{Z},<,+)$, then $\mathcal{S}$ is interdefinable with $(\mathbb{R},<,+, \mathbb{Z})$. It follows that any $(\mathbb{R},<,+, \mathbb{Z})$-definable subset of $\mathbb{R}^{n}$ is a finite union of sets of the the form $\bigcup_{b \in B} b+A$ for $(\mathbb{R},<,+)$-definable $A \subseteq[0,1)^{n}$ and $(\mathbb{Z},<,+)$ definable $B \subseteq \mathbb{Z}^{n}$. This description of definable sets previously appeared in work by computer scientists $[8,10$.

If $\alpha=\pi, \mathcal{J}$ is $\left([0, \pi),<,+{ }_{\pi},\left.\sin \right|_{[0, \pi)}\right)$, and $\mathcal{D}$ is $(\pi \mathbb{Z},<,+)$, then $\mathcal{S}$ is interdefinable with $\left(\mathbb{R},<,+\right.$, sin). More generally, suppose that $f: \mathbb{R} \rightarrow \mathbb{R}^{n}$ is analytic and $\alpha>0$ is such that $f(t+\alpha)=f(t)$ for all $t$. Letting $\mathcal{J}$ be $\left([0, \alpha),<,+_{\alpha},\left.f\right|_{[0, \alpha)}\right)$ and $\mathcal{D}$ be $(\alpha \mathbb{Z},<,+)$, we easily see that $(\mathbb{R},<,+, f)$ is interdefinable with $\mathcal{S}$. O-minimality of $\mathbb{R}_{\mathrm{an}}$ (see [84]) implies $\mathcal{J}$ is o-minimal, so $(\mathbb{R},<,+, f)$ is locally o-minimal.

Fact 2.1 shows in particular that if $\mathcal{D}$ is an NIP expansion of $(\mathbb{Z},<,+)$ then the expansion of $(\mathbb{R},<,+, \mathbb{Z})$ by all $\mathcal{D}$-definable subsets of all $\mathbb{Z}^{n}$ is NIP and locally o-minimal. Note that this structure is generated by closed sets. If $\mathcal{D}$ is $(\alpha \mathbb{Z},<,+)$ then $\mathcal{S}$ is strongly dependent as a disjoint union of two strongly dependent structures is strongly dependent.

Fact 2.1 also shows that the expansion of $(\mathbb{R},<,+)$ by all subsets of all $\mathbb{Z}^{n}$ is locally o-minimal. This is a special case of a general phenomenon uncovered by Miller and Friedman [30: geometric tameness properties for definable sets weaker then o-minimality do not imply any model-theoretic tameness.

It is easy to see that an expansion of $(\mathbb{R},<,+, \times)$ is locally o-minimal if and only if it is o-minimal. The basic example of an NIP expansion by closed sets which is not o-minimal is $\left(\mathbb{R},<,+, \times, \lambda^{\mathbb{Z}}\right)$ where $\lambda$ is a positive real other than 1 and

$$
\lambda^{\mathbb{Z}}:=\left\{\lambda^{i}: i \in \mathbb{Z}\right\} .
$$

This structure was first studied by van den Dries [83]. It is NIP by 34, Theorem 6.5]. Fact 2.2 follows by combining a theorem of Hieronymi and Miller [40] with a metric result of García, Hare, and Mendivil [31, Proposition 3.1] (see also Fraser and $\mathrm{Yu}$ [28, Theorem 6.1]).

Fact 2.2. Suppose $\left(s_{n}\right)_{n \in \mathbb{N}}$ is an eventually increasing sequence of positive real numbers such that $\left(s_{n+1}-s_{n}\right)_{n \in \mathbb{N}}$ is also eventually increasing. Let $S$ be $\left\{s_{n}: n \in\right.$ $\mathbb{N}\}$. If $(\mathbb{R},<,+, \times, S)$ does not define the set of integers then there is $\lambda>1$ such that $s_{n} \geq \lambda^{n}$ for sufficiently large $n$.

The assumption that $\left(s_{n+1}-s_{n}\right)_{n \in \mathbb{N}}$ is eventually increasing is necessary by Thamrongthanyalak [79]. It is a theorem of Miller and Speissegger that an expansion of $(\mathbb{R},<,+, \times)$ by closed sets is either o-minimal or defines an infinite closed and discrete subset of $\mathbb{R}$, see Fact 2.7 . So $\left(\mathbb{R},<,+, \times, \lambda^{\mathbb{Z}}\right)$ is arguably the most natural example of a non o-minimal tame expansion of $(\mathbb{R},<,+, \times)$ by a closed set. 
The expansion $\left(\mathbb{R},<,+, \times, \lambda^{\mathbb{Z}}\right)$ is a special case of an interesting family of expansions. Let $\mathcal{S}$ be an o-minimal expansion of $(\mathbb{R},<,+, \times)$. We say that $\mathcal{S}$ has rational exponents if the function $\mathbb{R} \rightarrow \mathbb{R}$ given by $t \mapsto t^{r}$ is only definable when $r \in \mathbb{Q}$. Hieronymi 37] showed that $\left(\mathbb{R},<,+, \times, \lambda^{\mathbb{Z}}, \eta^{\mathbb{Z}}\right)$ defines the set of integers for any $\lambda, \eta>1$ such that $\log _{\lambda} \eta \notin \mathbb{Q}$. So if $\mathcal{S}$ does not have rational exponents then $\left(\mathcal{S}, \lambda^{\mathbb{Z}}\right)$ defines the set of integers. Miller and Speissegger showed that if $\mathcal{S}$ has rational exponents then $\left(\mathcal{S}, \lambda^{\mathbb{Z}}\right)$ admits quantifier elimination in a natural expanded language [63, Section 8.6]. Tychonievich studied $\left(\mathcal{S}, \lambda^{\mathbb{Z}}\right)$ in his thesis [82. It follows from [82, Theorem 4.1.2, Corollary 4.1.7] and work of Chernikov and Simon [13, Corollary 2.6] that $\left(\mathcal{S}, \lambda^{\mathbb{Z}}\right)$ is NIP when $\mathcal{S}$ has rational exponents. Let $\mathbf{e}$ and $\mathbf{s}$ be the restrictions of the exponential function and $\sin$ to $[0,2 \pi]$, respectively. Then $(\mathbb{R},<,+, \times, \mathbf{e}, \mathbf{s})$ is o-minimal and has rational exponents by van den Dries [84. It is observed in [63, Section 3.4] that $\left(\mathbb{R},<,+, \times, \mathbf{e}, \mathbf{s}, \lambda^{\mathbb{Z}}\right)$ defines the logarithmic spiral

$$
\left\{\left(e^{t} \sin (\alpha t), e^{t} \cos (\alpha t)\right): t \in \mathbb{R}\right\}
$$

where $\lambda=e^{2 \pi \alpha}$. So the expansion of $(\mathbb{R},<,+, \times)$ by a logarithmic spiral is an NIP expansion by a closed set which is not locally o-minimal.

It should be noted that $\left(\mathcal{S}, \lambda^{\mathbb{Z}}\right)$ satisfies a stronger condition then generic local ominimality. If $\mathcal{S}$ has rational exponents then $\left(\mathcal{S}, \lambda^{\mathbb{Z}}\right)$ is $\mathbf{d}$-minimal: every unary definable set in every model of the theory of $\left(\mathcal{S}, \lambda^{\mathbb{Z}}\right)$ is a union of an open set together with finitely many discrete sets. In particular every nowhere dense definable subset of $\mathbb{R}$ has finite Cantor rank. Question 2.3 is open, we expect it to have a positive answer. (However, we expect that natural examples of NIP expansions of $(\mathbb{R},<,+)$ have d-minimal open core.)

Question 2.3. Is there an NIP expansion of $(\mathbb{R},<,+)$ which defines a nowhere dense subset of $\mathbb{R}$ with infinite Cantor rank? Is there an NIP expansion of $(\mathbb{R},<,+)$ which defines an uncountable nowhere dense subset of $\mathbb{R}$ ?

Finally, it is worth pointing out that there are well-behaved expansions of $(\mathbb{R},<,+)$ which are noiseless and not strongly noiseless. Theorem 2.17 shows that a noiseless expansion is strongly noiseless if and only if it does not define a Cantor subset of $\mathbb{R}$. Friedman, Kurdyka, Miller, and Speissegger [29] gave the first example of a Cantor subset $K$ of $\mathbb{R}$ such that $(\mathbb{R},<,+, \times, K)$ is noiseless. Hieronymi 39] shows that if $\mathcal{S}$ is an o-minimal expansion of $(\mathbb{R},<,+, \times)$ such that every $\mathcal{S}$-definable function $\mathbb{R} \rightarrow \mathbb{R}$ is eventually bounded above by some compositional iterate of the exponential (every known o-minimal expansion of the real field satisfies this condition), then there is a Cantor subset $K$ of $\mathbb{R}$ such that $(\mathcal{S}, K)$ is model-theoretically tame and noiseless.

2.2. The core conjectures. A subset of $\mathbb{R}^{k}$ is $\omega$-orderable if it is definable and is either finite or admits a definable ordering with order type $\omega$. One should think of " $\omega$-orderable" as "definably countable". A dense $\omega$-order is an $\omega$-orderable subset of $\mathbb{R}$ which is dense in some nonempty open interval. The presence or absence of a dense $\omega$-order has remarkably strong consequences. Hieronymi's Theorem is equivalent to the main theorem of [37. The left to right implication follows as any countable subset of $\mathbb{R}^{n}$ is $\omega$-orderable in $(\mathbb{R},<,+, \times, \mathbb{Z})$.

Fact 2.3 (Hieronymi's Theorem). Suppose $\mathcal{R}$ expands $(\mathbb{R},<,+, \times)$. Then there is a dense $\omega$-order if and only if the set $\mathbb{Z}$ of integers is definable. 
Fact 2.4 is proven in Hieronymi and Walsberg 43 .

Fact 2.4. Suppose that $\mathcal{R}$ defines at least one of the following:

(1) an unbounded continuous function $I \rightarrow \mathbb{R}^{n}$ on a bounded interval $I$,

(2) a non-affine $C^{2}$-function $U \rightarrow \mathbb{R}^{n}$ on a connected open subset $U$ of $\mathbb{R}^{m}$,

(3) the function $[0,1] \rightarrow \mathbb{R}$ given by $x \mapsto \lambda x$ for uncountably many $\lambda \in \mathbb{R}$.

Then there is a dense $\omega$-order if and only if every bounded Borel subset of every $\mathbb{R}^{n}$ is definable.

Fact 2.5 is due to Hieronymi and Walsberg 44 . We consider the two-sorted first order structure $(\mathcal{P}(\mathbb{N}), \mathbb{N}, \in s)$ where $\mathcal{P}(\mathbb{N})$ is the power set of $\mathbb{N}$ and $s: \mathbb{N} \rightarrow \mathbb{N}$ is the usual successor function. We identify the (first order) theory of $(\mathcal{P}(\mathbb{N}), \mathbb{N}, \in, s)$ with the monadic second order theory of $(\mathbb{N}, s)$.

Fact 2.5. If $\mathcal{R}$ admits a dense $\omega$-order then $\mathcal{R}$ defines an isomorphic copy of $(\mathcal{P}(\mathbb{N}), \mathbb{N}, \in, s)$.

Fact 2.5 is sharp in that $(\mathcal{P}(\mathbb{N}), \mathbb{N}, \in, s)$ defines isomorphic copies of first order expansions of $(\mathbb{R},<,+)$ which admit dense $\omega$-orders. Recall that the theory of $(\mathcal{P}(\mathbb{N}), \mathbb{N}, \in, s)$ is decidable by a theorem of Büchi [11].

We say that an expansion of $(\mathbb{R},<,+)$ is type $\mathbf{A}$ if there are no dense $\omega$-orders. Conjecture 1 is the main conjecture on first order expansions of $(\mathbb{R},<,+)$.

Conjecture 1. If $\mathcal{R}$ is type $A$ then $\mathcal{R}^{\circ}$ is noiseless. In particular if $\mathcal{R}$ expands $(\mathbb{R},<,+, \times)$ and does not define $\mathbb{Z}$ then $\mathcal{R}^{\circ}$ is noiseless.

We recall another theorem from [44].

Fact 2.6. If $\mathcal{R}$ defines a Cantor subset of $\mathbb{R}$ then $\mathcal{R}$ defines an isomorphic copy of $(\mathcal{P}(\mathbb{N}), \mathbb{N}, \in, s)$.

Fact 2.6 is sharp as $(\mathcal{P}(\mathbb{N}), \mathbb{N}, \in, s)$ defines an isomorphic copy of $(\mathbb{R},<,+, K)$ where $K$ is the classical middle-thirds Cantor set [9, Theorem 5]. In Theorem 2.17 we give a proof of the fact, well known to experts, that an expansion of $(\mathbb{R},<,+)$ is generically locally o-minimal if and only if it is noiseless and does not define a Cantor subset of $\mathbb{R}$. So Conjecture 2 is a special case of Conjecture 1 .

Conjecture 2. If $\mathcal{R}$ does not define an isomorphic copy of $\left(\mathcal{P}(\mathbb{N}), \mathbb{N}, \in\right.$, s) then $\mathcal{R}^{\circ}$ is generically locally o-minimal.

We now describe several known cases of Conjecture 1. The following theorem of Miller and Speissegger [65] is a special case of Conjecture 1]

Fact 2.7. Suppose $\mathcal{R}$ expands $(\mathbb{R},<,+, \times)$. Then $\mathcal{R}$ does not define an infinite closed discrete subset of $\mathbb{R}$ if and only if $\mathcal{R}^{\circ}$ is o-minimal.

It follows from the proof of [44, Theorem $\mathrm{A}$ ] that if $\mathcal{R}$ admits a dense $\omega$-order then $\mathcal{R}$ defines an infinite bounded discrete subset of $\mathbb{R}$. So Fact 2.8 is another special case of Conjecture 1 Fact 2.8 will be proven in forthcoming joint work with Hieronymi.

Fact 2.8. Every $\mathcal{R}$-definable nowhere dense subset of $\mathbb{R}$ is closed and discrete if and only if $\mathcal{R}^{\circ}$ is locally o-minimal.

The following theorem of Fornasiero, Hieronymi, and Walsberg [27, Theorem D] shows in particular that if $\mathcal{R}$ is type A then every subset of $\mathbb{R}^{m}$ which is existentially definable in $\mathcal{R}^{\circ}$ either has interior or is nowhere dense. 
Fact 2.9. Suppose that $\mathcal{R}$ is type A. Let $X$ be a definable constructible subset of $\mathbb{R}^{n}$. If $f: X \rightarrow \mathbb{R}^{m}$ is definable and continuous then $f(X)$ either has interior or is nowhere dense.

Theorem 2.10 is known to experts but has not previously been stated in full generality. It shows that a type A expansion by zero-dimensional closed subsets of $\mathbb{R}^{n}$ and closed subsets of $\mathbb{R}$ is noiseless.

Theorem 2.10. Suppose $\mathcal{S}$ is an o-minimal expansion of $(\mathbb{R},<,+)$. Let $\mathcal{C}$ be a collection of subsets of Euclidean space such that the closure of each $X \in \mathcal{C}$ is zero-dimensional and let $\mathcal{D}$ be a collection of subsets of $\mathbb{R}$ such that each $C \in \mathcal{D}$ is not dense and co-dense in any nonempty open interval. If $(\mathcal{S}, \mathcal{C}, \mathcal{D})$ is type $A$ then $(\mathcal{S}, \mathcal{C}, \mathcal{D})$ is noiseless.

Theorem 2.10 is an application of the next two facts. The first, a theorem of Friedman and Miller [30, requires some notation. If $\mathcal{S}$ is a first order expansion of $(\mathbb{R},<,+)$ and $E$ is a subset of $\mathbb{R}$ then $(\mathcal{S}, E)^{\sharp}$ is the expansion of $\mathcal{S}$ by all subsets of all cartesian powers of $E$. Note that if $E$ is infinite then $(\mathcal{S}, E)^{\sharp}$ defines an isomorphic copy of $(\mathbb{Z},+, \times)$ and if $E$ is uncountable then $(\mathcal{S}, E)^{\sharp}$ defines an isomorphic copy of the standard model of second order arthimetic. So Fact 2.11 may be a surprise.

Fact 2.11. Let $\mathcal{S}$ be an o-minimal expansion of $(\mathbb{R},<,+)$ and $E$ be a subset of $\mathbb{R}$. If $f\left(E^{n}\right)$ is nowhere dense for every $\mathcal{S}$-definable $f: \mathbb{R}^{n} \rightarrow \mathbb{R}$ then $(\mathcal{S}, E)^{\sharp}$ is noiseless.

Miller and Friedman only show that $(\mathcal{S}, E)^{\sharp}$ is noiseless in one variable. Fact 2.16 below shows that $(\mathcal{S}, E)^{\sharp}$ is noiseless. Fact 2.12 follows from [27, Proposition 5.6].

Fact 2.12. Suppose $\mathcal{R}$ is type $A$. Let $X$ be a constructible definable subset of $\mathbb{R}^{n}$ and $f: X \rightarrow \mathbb{R}^{m}$ be continuous and definable. If $X$ is zero-dimensional then $f(X)$ is nowhere dense.

We may now prove Theorem 2.10 .

Proof. Given $1 \leq k \leq n$ we let $\pi_{k}^{n}: \mathbb{R}^{n} \rightarrow \mathbb{R}$ be the map given by

$$
\pi_{k}^{n}\left(x_{1}, \ldots, x_{n}\right)=x_{k} .
$$

We let $\mathcal{R}$ be $(\mathcal{S}, \mathcal{C}, \mathcal{D})$. We suppose that $\mathcal{R}$ is type $\mathrm{A}$. Note that $\mathcal{R}$ is noiseless if and only if $\left(\mathcal{S}, \mathcal{C}^{\prime}, \mathcal{D}^{\prime}\right)$ is noiseless for any finite subcollection $\mathcal{C}^{\prime}$ of $\mathcal{C}$ and finite subcollection $\mathcal{D}^{\prime}$ of $\mathcal{D}$. So we assume $\mathcal{C}$ and $\mathcal{D}$ are finite. Suppose that $\mathcal{R}$ is type A. We now define an auxiliary collection $\mathcal{E}$ of subsets of $\mathbb{R}$ consisting of:

- $\pi_{k}^{n}(\mathrm{Cl}(X))$ for all $1 \leq k \leq n$ and $X \in \mathcal{C}$ such that $X \subseteq \mathbb{R}^{n}$,

- $\operatorname{Bd}(X)$ for all $X \in \mathcal{D}$.

If $X \in \mathcal{D}$ then $\operatorname{Bd}(X)$ is nowhere dense as $X$ is not dense and co-dense in any open interval. If $X \in \mathcal{C}$, then $\mathrm{Cl}(X)$ is zero-dimensional by assumption, so each $\pi_{k}^{n}(\mathrm{Cl}(X))$ is nowhere dense by Fact 2.12. So every element of $\mathcal{E}$ is nowhere dense. Note also that $\mathcal{E}$ is finite as $\mathcal{C}$ and $\mathcal{D}$ are finite, so $\bigcup \mathcal{E}$ is nowhere dense. Let $E$ be the closure of $\bigcup \mathcal{E}$, so $E$ is closed, nowhere dense, and definable.

We show that $E$ satisfies the condition of Fact 2.11. Let $f: \mathbb{R}^{n} \rightarrow \mathbb{R}$ be $\mathcal{S}$-definable. We show that $f\left(E^{n}\right)$ is nowhere dense. Applying o-minimal cell decomposition we obtain a finite partition $\mathcal{F}$ of $\mathbb{R}^{n}$ into $\mathcal{S}$-definable cells such that the restriction of $f$ to each element of $\mathcal{F}$ is continuous. Fix $Z \in \mathcal{F}$. As $E$ is closed and zero-dimensional, 
$E^{n}$ is also closed and zero-dimensional. So $Z \cap E^{n}$ is zero-dimensional. It easily follows from the definition of a cell that any cell is constructible, so $Z$ is constructible. So $Z \cap E^{n}$ is constructible. An application of Fact 2.12 now shows that $f\left(Z \cap E^{n}\right)$ is nowhere dense. So $f\left(E^{n}\right)$ is a finite union of nowhere dense sets and is hence nowhere dense. Fact 2.11 shows that $(\mathcal{S}, E)^{\sharp}$ is noiseless.

It now suffices to show that every element of $\mathcal{C}$ and $\mathcal{D}$ are $(\mathcal{S}, E)^{\sharp}$-definable. Every element of $\mathcal{E}$ is a subset of $E$ and is thus $(\mathcal{S}, E)^{\sharp}$-definable. Fix $X \in \mathcal{C}$ and suppose $X \subseteq \mathbb{R}^{n}$. Then

$$
X \subseteq \prod_{k=1}^{n} \pi_{k}^{n}(\mathrm{Cl}(X)) \subseteq E^{n}
$$

so $X$ is $(\mathcal{S}, E)^{\sharp}$-definable. Now suppose $X \in \mathcal{D}$. Let $U$ be the interior of $X$. Then

$$
X \backslash U \subseteq \operatorname{Bd}(X) \subseteq E
$$

so $X \backslash U$ is $(\mathcal{S}, E)^{\sharp}$-definable. We show that $U$ is $(\mathcal{S}, E)^{\sharp}$-definable. Let $A$ be the set of $(a, b) \in \operatorname{Bd}(X)^{2}$ such that $a<b$ and the open interval with endpoints $a, b$ is contained in $U$. Then $A \subseteq E^{2}$ and so $A$ is $(\mathcal{S}, E)^{\sharp}$-definable. Finally

$$
U=\bigcup_{(a, b) \in A}\{t \in \mathbb{R}: a<t<b\}
$$

so $U$ is $(\mathcal{S}, E)^{\sharp}$-definable.

2.3. Local and generic local o-minimality. Theorem 2.13 gives several equivalent definitions of local o-minimality for expansions of $(\mathbb{R},<,+)$. Locally o-minimal structures are studied in 80, 51. In particular the equivalence of (2) and (3) below was proven in 80 .

Theorem 2.13. The following are equivalent:

(1) $\mathcal{R}$ is locally o-minimal,

(2) for every definable $X \subseteq \mathbb{R}$ and $a \in \mathbb{R}$ there is an open interval I containing a such that $I \cap X$ is $(\mathbb{R},<)$-definable,

(3) the structure induced by $\mathcal{R}$ on any bounded interval I is o-minimal,

(4) the expansion of $(\mathbb{R},<,+)$ by all bounded $\mathcal{R}$-definable sets is o-minimal,

$(5) \mathcal{R}$ is noiseless and does not define a bounded discrete subset of $D$ of $\mathbb{R}_{>0}$ such that $\mathrm{Cl}(D)=D \cup\{0\}$,

(6) $\mathcal{R}$ is noiseless and every nowhere dense definable subset of $\mathbb{R}$ is closed and discrete.

We will need four results for the proof of Theorem 2.13. The first is an easy fact about subsets of $\mathbb{R}$ whose verification we leave to the reader.

Fact 2.14. Suppose $I$ is an open interval and $X$ is a subset of $I$. Then $X$ is a finite union of open intervals and singletons if and only if $\operatorname{Bd}(X) \cap I$ is finite.

We say that $\mathcal{R}$ is o-minimal at infinity if for every definable $X \subseteq \mathbb{R}$ there is $t>0$ such that $(t, \infty)$ is either contained in or disjoint from $X$. Fact 2.15 is a special case of a theorem of Belegradek, Verbovskiy, and Wagner [4, Theorem 19].

Fact 2.15. The expansion of $(\mathbb{R},<,+)$ by all bounded subsets of all $\mathbb{R}^{n}$ is o-minimal at infinity.

Fact 2.16] is due to Miller [63, Theorem 3.2]. 
Fact 2.16. $\mathcal{R}$ is noiseless if and only if it is noiseless is one variable.

We now prove Theorem 2.13 .

Proof. (2) $\Rightarrow(3)$ : Let $I$ be a bounded interval. After replacing $I$ with its closure if necessary we suppose $I$ is closed. Fix a definable subset $X$ of $I$. We show that $X$ is definable in $(\mathbb{R},<)$. For every $a \in I$ let $J_{a}$ be an open interval containing $a$ such that $J_{a} \cap X$ is definable in $(\mathbb{R},<)$. As $I$ is compact there is a finite $A \subseteq I$ such that $\left(J_{a}\right)_{a \in A}$ covers $I$. Then

$$
X=\bigcup_{a \in A} J_{a} \cap X
$$

so $X$ is definable in $(\mathbb{R},<)$.

$(3) \Rightarrow(4)$ : Let $\mathcal{B}$ be the collection of all bounded definable sets. Suppose $X \subseteq \mathbb{R}$ is definable in $(\mathbb{R},<,+, \mathcal{B})$. Fact 2.15 yields a $t>0$ such that $X \backslash[-t, t]$ is definable in $(\mathbb{R},<)$. The induced structure on $[-t, t]$ is o-minimal, so $X \cap[-t, t]$ is definable in $(\mathbb{R},<)$. So $X$ is $(\mathbb{R},<)$-definable.

$(4) \Rightarrow(5)$ : Suppose that $\mathcal{R}$ is noisey. Let $X$ be a definable subset of $\mathbb{R}^{n}$ which is dense and co-dense in a nonempty definable open subset $U$ of $\mathbb{R}^{n}$. We may suppose that $U$ is bounded. Then $U \cap X$ is bounded and definable and $(\mathbb{R},<,+, U \cap X)$ is not o-minimal, contradiction. Suppose that $D$ is a bounded discrete subset of $\mathbb{R}_{>0}$ such that $\mathrm{Cl}(D)=D \cup\{0\}$. Then $\operatorname{Bd}(D)=D \cup\{0\}$ is infinite, an application of Fact 2.14 shows that $(\mathbb{R},<,+, D)$ is not o-minimal.

$(5) \Rightarrow(6)$ : Suppose that $X$ is a nowhere dense definable subset of $\mathbb{R}$ which is not closed and discrete. Fix a bounded open interval $I$ such that $I \cap X$ is infinite. Note that each connected component of $I \backslash \mathrm{Cl}(X)$ is a nonempty open interval. We let $D$ be the set of lengths of connected components of $I \backslash \mathrm{Cl}(X)$. Then $D$ is infinite as $\mathrm{Cl}(X)$ is infinite and bounded as $I$ is bounded. As $I$ is bounded there are only finitely many connected components of length $>t$ for any $t>0$. Thus $D \backslash(0, t)$ is finite for any $t>0$. It follows that $D$ is discrete and $\operatorname{Cl}(D)=D \cup\{0\}$.

$(6) \Rightarrow(2)$ : Fix a definable subset $X$ of $\mathbb{R}$ and $a \in X$. Then $\operatorname{Bd}(X)$ is nowhere dense as $X$ is nowhere dense and co-dense. So $\operatorname{Bd}(X)$ is closed and discrete. Let $I$ be a bounded open interval containing $a$ such that $I \cap \operatorname{Bd}(X)$ is finite. Fact 2.14 shows that $I \cap X$ is definable in $(\mathbb{R},<)$.

It is immediate that (2) implies (1). We show that (1) implies (6). Let $X \subseteq \mathbb{R}$ be definable and somewhere dense and $I$ be a nonempty open interval in which $X$ is dense. Fix $p \in I \cap X$ and a subinterval $J$ of $I$ such that $J \cap X$ is $(\mathbb{R},<)$-definable. As $X$ is dense in $J, J \cap X$ is cofinite, so $X$ has interior. Now suppose that $Y \subseteq \mathbb{R}$ is nowhere dense and definable. It suffices to show that every $p \in \mathrm{Cl}(X)$ is isolated. Let $I$ be a open interval containing $p$ such that $I \cap \mathrm{Cl}(X)$ is $(\mathbb{R},<)$-definable. As $\mathrm{Cl}(X)$ is nowhere dense, $I \cap \mathrm{Cl}(X)$ is finite hence $p$ is isolated in $I \cap \mathrm{Cl}(X)$.

Theorem 2.17 gives conditions that are equivalent to generic local o-minimality.

Theorem 2.17. The following are equivalent:

(1) $\mathcal{R}$ is generically locally o-minimal, 
(2) every definable subset of $\mathbb{R}$ either has interior or contains an isolated point,

(3) $\mathcal{R}$ is strongly noiseless,

(4) the structure induced on $[0,1]$ by $\mathcal{R}$ is strongly noiseless,

(5) $\mathcal{R}$ is strongly noiseless in one variable,

(6) $\mathcal{R}$ is noiseless in one variable and does not define a Cantor subset of $\mathbb{R}$.

We need another result of Miller. Fact 2.18 follows from [63, Proposition 3.4].

Fact 2.18. Suppose every $\mathcal{R}$-definable subset of $\mathbb{R}$ either has interior or contains an isolated point. Let $X$ be a nonempty $\mathcal{R}$-definable subset of $\mathbb{R}^{n}$. Then there is a dense open subset $V$ of $X$ such that for every $p \in V$ there is $0 \leq d \leq n$, a coordinate projection $\pi: \mathbb{R}^{n} \rightarrow \mathbb{R}^{d}$, and an open neighbourhood $W$ of $p$ such that $\pi(X \cap W)$ is open and $\pi$ induces a homeomorphism $X \cap W \rightarrow \pi(X \cap W)$.

We now prove Theorem 2.17

Proof. We first show that (1) and (2) are equivalent. Let $X \subseteq \mathbb{R}$ be definable. Suppose $\mathcal{R}$ is generically locally o-minimal. Then there is a $p \in X$ and an open interval $I$ containing $p$ such that $I \cap X$ is $(\mathbb{R},<)$ definable. So $I \cap X$ either has interior or is finite and hence contains an isolated point. Suppose (2) holds. Let $U$ be the interior of $X$ and $D$ be the set of isolated points of $X$. Note that $U \cup D$ is open in $X$. It suffices to show that $U \cap D$ is dense in $X$. Suppose otherwise. Then there is $p \in X$ and an open interval $I$ containing $p$ such that $I$ is disjoint from $U \cap D$. Then $I \cap X$ does not have interior and does not contain an isolated point, contradiction.

$(2) \Rightarrow(3)$ : Note that $(2)$ implies that $\mathcal{R}$ is noiseless in one variable, so $\mathcal{R}$ is noiseless by Fact 2.16. Let $X, Y$ be definable subsets of $\mathbb{R}^{n}$. Suppose that $X$ is somewhere dense in $Y$. Fix a nonempty definable open subset $W$ of $Y$ such that $X$ is dense in $W \cap Y$. After replacing $Y$ with $W \cap Y$ if necessary we suppose $X$ is dense in $Y$. Applying Fact 2.18 we obtain $0 \leq d \leq n$, definable open $U \subseteq \mathbb{R}^{n}, V \subseteq \mathbb{R}^{d}$, and a coordinate projection $\pi: \mathbb{R}^{n} \rightarrow \mathbb{R}^{d}$ such that $\pi$ restricts to a homeomorphism $U \cap Y \rightarrow V$. Then $\pi(X \cap U)$ is dense in $V$. So $\pi(X \cap U)$ has interior in $V$ as $\mathcal{R}$ is noiseless. It follows that $X$ has interior in $U \cap Y$ and thus has interior in $Y$.

It is clear that (3) implies (4). We show that (4) implies (5). Suppose $\mathcal{R}$ is not strongly noiseless in one variable. Let $X, Y$ be definable subsets of $\mathbb{R}$ such that $X$ is somewhere dense in $Y$ and has empty interior in $Y$. Let $p$ be an element of $X$ and $I$ be an open interval containing $p$ such that $I \cap X$ is dense in $I \cap Y$. Then $\left(X-p+\frac{1}{2}\right) \cap[0,1]$ is somewhere dense in $\left(Y-p+\frac{1}{2}\right) \cap[0,1]$ but $\left(X-p+\frac{1}{2}\right) \cap[0,1]$ has empty interior in $\left(Y-p+\frac{1}{2}\right) \cap[0,1]$. So the structure induced on $[0,1]$ by $\mathcal{R}$ is not strongly noiseless.

$(5) \Rightarrow(6)$ : It suffices to suppose there is a definable Cantor set and show that (5) fails. Let $Y$ be a definable Cantor set. Observe that each connected component of $\mathbb{R} \backslash Y$ is an interval. Let $X$ be the set of endpoints of connected components of $\mathbb{R} \backslash Y$. Note $X$ is a definable subset of $Y$. It is easy to see that $X$ is dense and co-dense in $Y$.

$(6) \Rightarrow(2)$ : Let $X$ be a definable subset of $\mathbb{R}$. We show that $X$ either has interior or contains an isolated point. If $X$ is somewhere dense then $X$ has interior. Suppose $X$ is nowhere dense. It suffices to show that $\mathrm{Cl}(X)$ has an isolated point. After 
replacing $X$ by its closure we suppose $X$ is closed nowhere dense. As $X$ is totally disconnected there is a bounded open interval $I$ such that $I \cap X \neq \emptyset$ and $\operatorname{Bd}(I) \cap X=$ $\emptyset$. So $I \cap X$ is nonempty, closed, bounded, and nowhere dense. As $I \cap X$ is not a Cantor set, it has an isolated point.

2.4. D-minimality and Pillay rank. At several points we will need to assume d-minimality. We introduce a useful tool in this setting, the Pillasy rank. We say that $\mathcal{R}$ satisfies the closed chain condition if there is no sequence $\left(X_{k}\right)_{k \in \mathbb{N}}$ of nonempty definable subsets of some $\mathbb{R}^{n}$ such that $X_{k+1}$ is a nowhere dense subset of $X_{k}$ for all $k$. It is easy to see that $\mathcal{R}$ fails the closed chain condition if and only if there is a sequence $\left\{X_{k}\right\}_{k \in \mathbb{N}}$ of nonempty closed definable subsets of some $\mathbb{R}^{n}$ such that $X_{k+1}$ has empty interior in $X_{k}$ for all $k$.

Theorem 2.19. Suppose $\mathcal{R}$ is d-minimal. Then $\mathcal{R}$ satisfies the closed chain condition.

Theorem 2.19 requires Fact 2.20 which is elementary and left to the reader. We let $\mathrm{Cb}(X)$ be the Cantor-Bendixson rank of a subset $X$ of $\mathbb{R}$.

Fact 2.20. Suppose $X$ is a nonempty subset of $\mathbb{R}$ with finite Cantor-Bendixson rank and $Y$ is a subset of $X$. If $\mathrm{Cb}(X)=\mathrm{Cb}(Y)$ then $Y$ has interior in $X$.

We introduce some notation for the proof of Theorem 2.19, Let $Y$ be a definable subset of $\mathbb{R}$. We let:

(1) $\Omega(Y)=-1$ when $Y=\emptyset$,

(2) $\Omega(Y)=\mathrm{Cb}(Y)$ when $Y$ is nonempty and nowhere dense, and

(3) $\Omega(Y)=\omega$ when $Y$ has interior.

If $Z \subseteq \mathbb{R}^{n}$ is definable then by d-minimality $\Omega\left(X_{a}\right)$ takes only finitely many values as $a$ ranges over $\mathbb{R}^{n-1}$ and $\left\{a \in \mathbb{R}^{n-1}: \Omega\left(X_{a}\right)=\eta\right\}$ is definable for all $\eta \in \mathbb{N} \cup\{-1, \omega\}$.

Theorem 2.19 also requires Lemma 10.18 below. Lemma 10.18 holds more generally for generically locally o-minimal expansions of $(\mathbb{R},<,+)$, so we prove it in Section 10. We now prove Theorem 2.19.

Proof. Suppose towards a contradiction that $\left(X_{k}\right)_{k \in \mathbb{N}}$ is a sequence of nonempty definable subsets of $\mathbb{R}^{n}$ such that $X_{k+1}$ is a nowhere dense subset of $X_{k}$ for all $k$. We apply induction on $n$.

Suppose $n=1$. Note that $X_{k}$ is nowhere dense in $\mathbb{R}$ when $k \geq 1$. By dminimality $X_{k}$ has finite Cantor-Bendixson rank for all $k \geq 1$. Fact 2.20 shows that $\mathrm{Cb}\left(X_{k+1}\right)<\mathrm{Cb}\left(X_{k}\right)$ for all $k \geq 1$. Contradiction.

Suppose $n \geq 2$ and let $\pi: \mathbb{R}^{n} \rightarrow \mathbb{R}^{n-1}$ be the projection away from the first coordinate. By induction it suffices to show that for every $k \in \mathbb{N}$ there is $m>k$ such that $\pi\left(X_{m}\right)$ is nowhere dense in $\pi\left(X_{k}\right)$. For the sake of simplicity we only treat the case when $k=0$ and set $X:=X_{0}$.

For all $m \geq 1$ and $\eta \in \mathbb{N} \cup\{-1, \omega\}$ we let

$$
B_{m}^{\eta}:=\left\{a \in \pi(X): \Omega\left(\left(X_{m}\right)_{a}\right)=\eta\right\} .
$$

Note that for each $m$ there are only finitely many $\eta$ such that $B_{m}^{\eta}$ is nonempty. For each $m \geq 1$ we let $\zeta(m)$ be the maximal $\eta \in \mathbb{N} \cup\{-1, \omega\}$ such that $B_{m}^{\eta}$ is 
somewhere dense in $\pi(X)$. Observe that $\pi\left(X_{m}\right)$ is nowhere dense in $\pi(X)$ if and only if $\zeta(m)=-1$. So it suffices to show that $\zeta(m+1)<\zeta(m)$ when $\zeta(m) \geq 0$.

As $\left(X_{m}\right)_{m \geq 1}$ is decreasing, $\left(\Omega\left(\left(X_{m}\right)_{a}\right)_{m \geq 1}\right.$ is decreasing for all $a \in \pi(X)$. It follows that $(\zeta(m))_{m \geq 1}$ is decreasing. Fix $m$ and suppose towards a contradiction that $\zeta(m) \geq 0$ and $\zeta(m+1)=\zeta(m)$. Applying strong noiselessness let $U$ be a nonempty definable open subset of $\pi(X)$ contained in $B_{m+1}^{\zeta(m)}$. So $\Omega\left(\left(X_{m+1}\right)_{a}\right)=\zeta(m)$ for all $a \in U$. Hence $\Omega\left(\left(X_{m+1}\right)_{a}\right)=\Omega\left(\left(X_{m}\right)_{a}\right)$ for all $a \in U$. Applying Fact 2.20 we see that $\left(X_{m+1}\right)_{a}$ has interior in $\left(X_{m}\right)_{a}$ for all $a \in U$. Lemma 10.18 now shows that $X_{m+1}$ has interior in $X_{m}$. Contradiction.

Suppose that $\mathcal{R}$ is d-minimal. Let $\prec_{n}$ be the partial order on definable subsets of $\mathbb{R}^{n}$ where $X \prec_{n} Y$ when $X$ is a nowhere dense subset of $Y$. Theorem 2.19 shows that each $\prec_{n}$ is well-founded. We refer to the ordinal rank associated to $\prec_{n}$ as Pillay rank as it was defined (in a more general setting) by Pillay [1]. We denote the Pillay rank of a definable subset $X$ of $\mathbb{R}^{n}$ by $\operatorname{Pr}(X)$. If $X$ is a definable subset of $\mathbb{R}^{n}$, then:

(1) $\operatorname{Pr}(X)=-1$ if and only if $X$ is empty,

(2) If $\delta$ is an ordinal or -1 , then $\operatorname{Pr}(X) \geq \delta+1$ if and only if there is a nowhere dense definable $X^{\prime} \subseteq X$ such that $\operatorname{Pr}\left(X^{\prime}\right) \geq \delta$,

(3) If $\delta$ is a limit ordinal then $\operatorname{Pr}(X) \geq \delta$ if and only if $\operatorname{Pr}(X) \geq \eta$ for all ordinals $\eta<\delta$,

(4) If $X \neq \emptyset$ then $\operatorname{Pr}(X)$ is the supremum of all ordinals $\delta$ such that $\operatorname{Pr}(X) \geq \delta$. We now give several facts about Pillay rank, still assuming that $\mathcal{R}$ is d-minimal. We will not make use of these facts so we do not prove them. Suppose $Y$ is a definable subset of $\mathbb{R}^{n}$. Then $\operatorname{Pr}(Y)=0$ if and only if $Y$ is discrete and nonempty. If $\operatorname{dim} Y=0$ then $\operatorname{Pr}(Y)=\mathrm{Cb}(Y)$. If $\mathcal{R}$ is locally o-minimal then $\operatorname{Pr}(Y)=\operatorname{dim} Y$. If $\mathcal{R}$ is d-minimal and not locally o-minimal then $\operatorname{Pr}(Y)=\Omega(Y)$ when $Y \subseteq \mathbb{R}$.

Fact 2.21 is well-known and may be proven via an easy induction on Pillay rank.

Fact 2.21. If $\mathcal{R}$ is d-minimal then every definable set is constructible.

Fact 2.22 is also well-known, we include a proof for the sake of completeness.

Fact 2.22. Suppose $\mathcal{R}$ is d-minimal, $X$ is a definable subset of $\mathbb{R}^{n}$, and $\operatorname{dim} X=0$. Then $X$ is countable.

Proof. Given $1 \leq k \leq n$ let $\pi_{k}: \mathbb{R}^{n} \rightarrow \mathbb{R}$ be the projection onto the $k$ th coordinate. Fact 2.12 shows that each $\pi_{k}(X)$ is nowhere dense. By d-minimality each $\pi_{k}(X)$ is countable. So $X$ is countable as $X$ is a subset of $\pi_{1}(X) \times \ldots \times \pi_{k}(X)$.

\section{NIP}

We recall relevant background on NIP structures. Let $\mathcal{M}$ be a possibly multi-sorted first order structure and $\mathcal{N}$ be a highly saturated elementary extension of $\mathcal{M}$.

We first discuss externally definable sets. A subset $X$ of $M^{x}$ is externally definable (in $\mathcal{M}$ ) if there is an $\mathcal{N}$-definable subset $Y$ of $N^{x}$ such that $X=M^{x} \cap Y$. It is a saturation exercise to see that the collection of externally definable sets does not depend on choice of $\mathcal{N}$. We first record a useful observation whose verification we leave to the reader. 
Fact 3.1. Suppose $\mathcal{M}$ is an expansion of a linear order. Then every convex subset of $M$ is externally definable in $\mathcal{M}$.

The Shelah expansion $\mathcal{M}^{\mathrm{Sh}}$ of $\mathcal{M}$ is the expansion of $\mathcal{M}$ by all externally definable subsets of all $M^{x}$. If $\mathcal{M}$ is one-sorted then $\mathcal{M}^{\text {Sh }}$ is the structure induced on $M$ by $\mathcal{N}$. The following theorem is due to Shelah [72. Chernikov and Simon [13, Corollary 1.10] give a simpler proof.

Fact 3.2. Suppose $\mathcal{M}$ is NIP. Then every $\mathcal{M}^{\mathrm{Sh}}$-definable set is externally definable in $\mathcal{M}$. It follows that $\mathcal{M}^{\mathrm{Sh}}$ is NIP when $\mathcal{M}$ is NIP and $\mathcal{M}^{\mathrm{Sh}}$ is strongly dependent when $\mathcal{M}$ is strongly dependent.

The one-sorted case of Fact 3.2 asserts that the structure induced on $M$ by $\mathcal{N}$ eliminates quantifiers. We record another theorem of Chernikov and Simon [14, Corollary 9]. The right to left implication is a saturation exercise and does not require NIP.

Fact 3.3. Suppose $\mathcal{M}$ is NIP. Let $X$ be a subset of $M^{x}$. Then $X$ is externally definable in $\mathcal{M}$ if and only if there is an $\mathcal{M}$-definable family of $\left(X_{a}\right)_{a \in M^{y}}$ of subsets of $M^{x}$ such that for every finite $A \subseteq X$ there is a $\in M^{y}$ such that $A \subseteq X_{a} \subseteq X$.

We will need the following final easy fact whose verification we leave to the reader.

Fact 3.4. Suppose $Y \subseteq N^{x}$ is externally definable in $\mathcal{N}$. Then $Y \cap M^{x}$ is externally definable in $\mathcal{M}$.

We will need to use honest definitions at one point. Given an $\mathcal{M}$-definable $Z \subseteq M^{x}$ we let $Z^{\prime}$ be the subset of $N^{x}$ defined by any formula defining $Z$. Suppose $X$ is an externally definable subset of $M^{x}$. Then an $\mathcal{N}$-definable subset $Y$ of $N^{x}$ is an honest definition of $X$ if $Y \cap M^{x}=X$ and $Y \subseteq Z^{\prime}$ for every $\mathcal{M}$-definable $Z \subseteq M^{x}$ such that $X \subseteq Z$. Taking complements, we see that if $Y$ is an honest definition of $X$ then $Y \cap Z^{\prime}=\emptyset$ for every $\mathcal{M}$-definable $Z \subseteq M^{x}$ such that $X \cap Z=\emptyset$. The following theorem is due to Chernikov and Simon [14, Proposition 1.6].

Fact 3.5. Suppose $\mathcal{M}$ is NIP. Then every externally definable subset of $M^{x}$ has an honest definition.

It is an exercise to show that Fact 3.2 follows from Fact 3.5 .

We will use dp-ranks in a couple places. We refer to [75, Chapter 4] for the definition and background information. A structure is dp-minimal if it has dp-rank at most one. Onshuus and Usvyatsov [68, Observation 3.8] show that the Shelah expansion of a dp-minimal structure is dp-minimal. Their proof yields Fact 3.6 .

Fact 3.6. If $\mathcal{M}$ is NIP, then the dp-rank of $\mathcal{M}^{\mathrm{Sh}}$ agrees with the dp-rank of $\mathcal{M}$.

Fact 3.7 is proven by Kaplan, Onshuus, and Usvyatsov [50].

Fact 3.7. Suppose $\mathcal{M}$ is NIP. Suppose $X, Y$ are $\mathcal{M}$-definable sets of dp-rank $m, n$, respectively. Then the $d p$-rank of $X \times Y$ is at most $m+n$.

\section{Uniform Structures and $\bigwedge$-DEFinable EQUiValence RELATIONS}

4.1. Uniform structures. We describe background from the classical theory of uniform structures. We regard a basis for a uniform structure, defined below, as 
a generalization of a pseudo-metric, and regard a uniform structure as a generalization of a pseudo-metric considered up to uniform equivalence. One can consider a uniform structure to be an "approximate equivalence relation". As $\bigwedge$-definable equivalence relations are approximated by definable relations, there is a natural connection between $\bigwedge$-definable equivalence relations and uniform structures.

Let $X$ be a set and declare $\Delta:=\{(x, x): x \in X\}$ Given $A, B \subseteq X^{2}$ we declare

$$
A^{-1}:=\left\{\left(x^{\prime}, x\right):\left(x, x^{\prime}\right) \in A\right\}
$$

and

$$
A \circ B:=\left\{\left(x, x^{\prime}\right): \exists y(x, y) \in A,\left(y, x^{\prime}\right) \in B\right\} .
$$

We say that $A \subseteq X^{2}$ is symmetric if $A^{-1}=A$. A basis for a uniform structure on $X$ is a collection $\mathcal{B}$ of subsets of $X^{2}$ satisfying the following conditions.

(1) Every element of $\mathcal{B}$ contains $\Delta$.

(2) Every element of $\mathcal{B}$ is symmetric.

(3) For all $U, U^{\prime} \in \mathcal{B}$ there is $V \in \mathcal{B}$ such that $V \subseteq U \cap U^{\prime}$.

(4) For every $U \in \mathcal{B}$ there is $V \in \mathcal{B}$ such that $V \circ V \subseteq U$.

Suppose $\mathcal{B}$ is a basis for uniform structure on $X$. The uniform structure $\overline{\mathcal{B}}$ on $X$ generated by $\mathcal{B}$ is the collection of all subsets of $X^{2}$ which contain some element of $\mathcal{B}$. Suppose $\mathcal{B}$ is a basis for a uniform structure on $X$. We associate a topology on $X$ to $\mathcal{B}$ by declaring a subset $A$ of $X$ to be open if and only if for every $a \in A$ there is $U \in \mathcal{B}$ such that $U_{a} \subseteq A$. This topology only depends on $\overline{\mathcal{B}}$. Note that $\left(U_{a}\right)_{U \in \mathcal{B}}$ is a neighbourhood basis for $a \in X$. (In general $U_{a}$ need not be an open neighbourhood of $a$.) We say that a uniform structure is Hausdorff if the associated topology is Hausdorff. It is easy to see that $\overline{\mathcal{B}}$ is Hausdorff if and only if $\bigcap \mathcal{B}=\Delta$.

We leave the following lemma as a exercise.

Lemma 4.1. Suppose $\mathcal{B}$ is a basis for a uniform structure on $X$. Let $\mathcal{C}$ be a collection of subsets of $X^{2}$ such that

(1) Every $V \in \mathcal{C}$ is symmetric,

(2) Every $V \in \mathcal{C}$ contains some $U \in \mathcal{B}$,

(3) $\mathcal{B}$ is a subfamily of $\mathcal{C}$.

Then $\mathcal{C}$ is a basis for $\overline{\mathcal{B}}$.

We recall the main examples of uniform structures. If $(X, d)$ is a pseudo-metric space then the collection of sets of the form $\left\{\left(x, x^{\prime}\right) \in X^{2}: d\left(x, x^{\prime}\right)<t\right\}$ for $t>0$ is a basis for a uniform structure on $X$ which induces the $d$-topology. If $G$ is a topological group and $\mathcal{U}$ is a neighbourhood basis for the identity then the collection $\left\{\left(g, g^{\prime}\right) \in G^{2}: g^{-1} g^{\prime} \in U\right\}, U$ ranging over $\mathcal{U}$, is a basis for a uniform structure on $G$ which induces the group topology. Suppose $\tau$ is a compact Hausdorff topology on $X$ and equipp $X^{2}$ with the associated product topology. Then the collection of all symmetric open subsets of $X^{2}$ containing $\Delta$ forms a basis for a uniform structure on $X$. This is the unique uniform structure on $X$ for which the associated topology is $\tau$. If $E$ is an equivalence relation on $X$ then $\{E\}$ is a basis for a uniform structure on $X$. Finally, the discrete uniform structure on $X$ is the uniform structure with basis $\{\Delta\}$. 
Suppose $\mathcal{C}$ is a basis for a uniform structure on a set $Y$. Let $f$ be a function $X \rightarrow Y$. Then $f$ is uniformly continuous if for every $U \in \mathcal{C}$ there is $V \in \mathcal{B}$ such that for all $(a, b) \in V$ we have $(f(a), f(b)) \in U$. If $f$ is uniformly continuous then $f$ is a continuous map between the topologies associated to $\mathcal{B}$ and $\mathcal{C}$. We say that $f$ is a uniform equivalence if $f$ is bijective and $f, f^{-1}$ are both uniformly continuous.

One can associate a quotient Hausdorff uniform structure to a general uniform structure in a canonical way. It is easy to see that $E:=\bigcap \mathcal{B}$ is an equivalence relation on $X$. Let $\pi$ be the quotient map $X \rightarrow X / E$. Given $U \in \mathcal{B}$ we let $\pi(U)$ to be the set of $\left(y, y^{\prime}\right) \in(X / E)^{2}$ such that there are $x \in \pi^{-1}(\{y\}), x^{\prime} \in \pi^{-1}\left(\left\{y^{\prime}\right\}\right)$ satisfying $\left(x, x^{\prime}\right) \in U$. Let $\pi(\mathcal{B}):=(\pi(U))_{U \in \mathcal{B}}$. Then $\pi(\mathcal{B})$ is a basis for a Hausdorff uniform structure on $X / E$. Note that $E$ and the uniform structure on $X / E$ depend only on $\overline{\mathcal{B}}$.

Suppose $Y$ is a subset of $X$. Then

$$
\left.\mathcal{B}\right|_{Y}:=\left\{U \cap Y^{2}: U \in \mathcal{B}\right\}
$$

is a basis for a uniform structure on $Y$ which we refer to as the induced uniform structure on $Y$.

We describe the product uniform structure on $X^{n}$. Given $U \in \mathcal{B}$ we let $U_{n}$ be the set of $\left(x, x^{\prime}\right) \in X^{n} \times X^{n}$ such that $\left(x_{k}, x_{k}^{\prime}\right) \in U$ for all $1 \leq k \leq n$ where $x=\left(x_{1}, \ldots, x_{n}\right)$ and $x^{\prime}=\left(x_{1}^{\prime}, \ldots, x_{n}^{\prime}\right)$. Then $\mathcal{B}_{n}:=\left\{U_{n}: U \in \mathcal{B}\right\}$ is a basis for a uniform structure on $X^{n}$ which induces the product topology on $X^{n}$.

Lemma 4.2 will be crucial below. Given a subset $A$ of $X$ and $U \in \mathcal{B}$ we let $A[U]$ be $\bigcup_{a \in A} U_{a}$. Note that $A$ lies in the interior of $A[U]$.

Lemma 4.2. Suppose $\mathcal{W}$ is a finite collection of nonempty open subsets of $X$ and $A \subseteq X$ is not dense in any $W \in \mathcal{W}$. Then there is $U \in \mathcal{B}$ such that $W \backslash A[U]$ has interior for all $W \in \mathcal{W}$.

Proof. Fix $W \in \mathcal{W}$. We show there is $U_{W} \in \mathcal{B}$ such that $W \backslash A\left[U_{W}\right]$ has interior. Fix an element $b$ of $W$ that does not lie in the closure of $A$. So there is $V \in \mathcal{B}$ such that $V_{b}$ is disjoint from $A$. Let $U_{W} \in \mathcal{B}$ be such that $U_{W} \circ U_{W} \subseteq V$. We show that $A\left[U_{W}\right]$ and $\left(U_{W}\right)_{b}$ are disjoint. Suppose $p$ lies in both $A\left[U_{W}\right]$ and $\left(U_{W}\right)_{b}$. As $p \in A\left[U_{W}\right]$ there is $a \in A$ such that $p \in\left(U_{W}\right)_{a}$. Then $(a, p) \in U_{W}$ and $(p, b) \in U_{W}$. So $(a, b) \in V$. Contradiction. So $b$ lies in the interior of $W \backslash A\left[U_{W}\right]$.

Now let $U \in \mathcal{B}$ satisfy $U \subseteq \bigcap_{W \in \mathcal{W}} U_{W}$. Then $A[U] \subseteq A\left[U_{W}\right]$ for all $W \in \mathcal{W}$ so $W \backslash A[U]$ has interior for all $W \in \mathcal{W}$.

We now suppose that $\mathcal{M}$ is a (possibly multisorted) structure. Given a definable set $X$, a (sub)definable basis for a uniform structure on $X$ is a (sub)definable family of sets forming a basis for a uniform structure on $X$. If $\mathcal{B}$ is a subdefinable basis for a uniform structure on $X$ then $\mathcal{B}_{n}$ is a subdefinable basis for the product uniform structure on $X^{n}$ and $\left.\mathcal{B}_{n}\right|_{Y}$ is a subdefinable basis for the induced uniform structure on a definable $Y \subseteq X$.

Lemma 4.3. Suppose $\mathcal{B}$ is a subdefinable basis for a uniform structure on an $\mathcal{M}$ definable set $X$. Then $E:=\bigcap \mathcal{B}$ is externally definable in $\mathcal{M}$. 
Proof. Suppose $\mathcal{B}$ is a subdefinable basis for $E$. Let $\phi(x, y)$ be a formula such that for every $U \in \mathcal{B}$ there is $a \in M^{x}$ such that $\phi\left(a, M^{y}\right)=U$. It suffices to show that the following partial $x$-type is consistent

$$
\{\phi(x, e): e \in E\} \cup\left\{\forall y[\phi(x, y) \rightarrow \phi(a, y)]: \phi\left(a, M^{y}\right) \in \mathcal{C}\right\} .
$$

So it suffices to show that for any $U_{1}, \ldots, U_{n} \in \mathcal{B}$ there is $a \in M^{x}$ such that

$$
E \subseteq \phi\left(a, M^{y}\right) \subseteq \bigcap_{k=1}^{n} U_{k} .
$$

As $\mathcal{B}$ is a basis for the uniform structure associated to $E$ there is $V \in \mathcal{B}$ such that $V \subseteq \bigcap_{k=1}^{n} U_{k}$. Let $a \in M^{x}$ satisfy $\phi\left(a, M^{y}\right)=V$.

Proposition 4.4. Suppose $\mathcal{M}$ is NIP. Suppose $X$ is an $\mathcal{M}$-definable set and $\mathcal{B}$ is a subdefinable basis for a uniform structure on $X$. Then $\mathcal{M}^{\text {Sh }}$ defines a basis for $\overline{\mathcal{B}}$.

Proof. Let $\left(B_{a}\right)_{a \in M^{x}}$ be a definable family of sets such that $\mathcal{B}$ is a subfamily of $\left(B_{a}\right)_{a \in M^{x}}$. Let $\mathcal{N}$ be a highly saturated elementary extension of $\mathcal{M}$. Given an $\mathcal{M}$-definable set $Y$ we let $Y^{\prime}$ be the $\mathcal{N}$-definable set defined by the same formula as $Y$. We also let $\left(B_{a}^{\prime}\right)_{a \in N^{x}}$ be the family of sets defined by the same formula as $\left(B_{a}\right)_{a \in M^{x}}$. Note that $\mathcal{B}^{\prime}:=\left\{U^{\prime}: U \in \mathcal{B}\right\}$ is a subdefinable (in $\mathcal{N}$ ) basis for a uniform structure on $X^{\prime}$. Let $F:=\bigcap \mathcal{B}^{\prime}$. Lemma 4.3 shows that $F$ is definable in $\mathcal{N}^{\text {Sh }}$. An application of saturation shows that if $Y$ is an $\mathcal{M}$-definable subset of $X^{2}$ then $F \subseteq Y^{\prime}$ if and only if $Y$ contains some $U \in \mathcal{B}$. Let $Z$ be the set of $a \in N^{x}$ such that $F \subseteq B_{a}^{\prime}$. Then $Z$ is definable in $\mathcal{N}^{\text {Sh }}$. Facts 3.2 and 3.4 together show that $Q:=Z \cap M^{x}$ is definable in $\mathcal{M}^{\mathrm{Sh}}$. Then $Q$ is the set of $a \in M^{x}$ such that $B_{a}$ contains some $U \in \mathcal{B}$. In particular $\mathcal{B}$ is a subfamily of $\left(B_{a}\right)_{a \in Q}$.

Let $C_{a}=B_{a} \cap B_{a}^{-1}$ for all $a \in Q$. Note that each $C_{a}$ is symmetric and contains some $U \in \mathcal{B}$. Note also that $\mathcal{B}$ is a subfamily of $\left(C_{a}\right)_{a \in Q}$. An application of Lemma 4.1 shows that $\left(C_{a}\right)_{a \in Q}$ is a basis for $\overline{\mathcal{B}}$.

Corollary 4.5 follows immediately from Proposition 4.4.

Corollary 4.5. Suppose $\mathcal{M}$ is NIP, $X$ is an $\mathcal{M}$-definable set, and there is a subdefinable basis for a uniform structure on $X$. Let $X$ be the structure induced on $X$ by $\mathcal{M}$. Then the open core $\mathcal{X}^{\circ}$ of $\mathcal{X}$ is a reduct of the structure induced on $X$ by $\mathcal{M}^{\mathrm{Sh}}$.

4.2. \-definable equivalence relations. We now describe how uniform structures arise in abstract model theory. In this section $\mathcal{N}$ is a highly saturated structure and $X$ is an $\mathcal{N}$-definable set. There is a canonical correspondence between

- $\Lambda$-definable equivalence relations $E$ on $X$, and

- uniform structures on $X$ which admit a basis $\mathcal{B}$ consisting of a small family of definable sets.

This correspondence is well-known to experts and used implicitly throughout the literature. If $\mathcal{B}$ is a small collection of definable subsets of $X^{2}$ forming a basis for a uniform structure on $X$ then $E$ is $\cap \mathcal{B}$. Note that $E$ is $\Lambda$-definable and only depends on $\overline{\mathcal{B}}$. Now suppose $E$ is a $\bigwedge$-definable equivalence relation on $X$. Let $\mathcal{C}$ be a small collection of definable subsets of $X$ such that $\cap \mathcal{C}=E$. After replacing $\mathcal{C}$ with

$$
\left\{C_{1} \cap \ldots \cap C_{n} \cap C_{1}^{-1} \cap \ldots \cap C_{n}^{-1}: C_{1}, \ldots, C_{n} \in \mathcal{C}\right\}
$$


if necessary we may suppose that every element of $\mathcal{C}$ is symmetric and that for all $C, C^{\prime} \in \mathcal{C}$ there is $B \in \mathcal{C}$ such that $B \subseteq C \cap C^{\prime}$. It is now an exercise in saturation to show that $\mathcal{C}$ is a basis for a uniform structure on $X$ and that this uniform structure does not depend on choice of $\mathcal{C}$. It is also a saturation exercise to see that the induced uniform structure on $X / E$ is the discrete uniform structure if and only if $E$ is definable. Finally, the quotient $X / E$ is small if and only if the induced topology on $X / E$ is compact. In this case the topology is known as the logic topology on $X / E$. A subset of $X / E$ is closed in the logic topology if and only if it is the image of a $\bigwedge$-definable subset of $X$ under the quotient map. In general we refer to the topology on $X / E$ as the logic topology.

Proposition 4.6. Let $E$ be $a \bigwedge$-definable equivalence relation on $X$ and $\pi$ be the quotient map $X \rightarrow X / E$. Then $\pi(Y)$ is closed for any definable $Y \subseteq X$.

Proof. Let $\mathcal{B}$ be a small collection of definable subsets of $X^{2}$ which forms a basis for the uniform structure on $X$. Let $Y$ be a definable subset of $X$. Suppose $p \in X / E$ lies in the closure of $\pi(Y)$. Fix $p^{\prime} \in X$ such that $\pi\left(p^{\prime}\right)=p$. For every $U \in \mathcal{B}$ there is $r_{U} \in Y$ such that $\left(p^{\prime}, r_{U}\right) \in U$. As $\mathcal{B}$ is small and $\cap \mathcal{B}=E$ an application of saturation yields an $r \in Y$ such that $\left(p^{\prime}, r\right) \in E$. So $\pi(r)=p$ and $p \in \pi(Y)$.

We also equipp $(X / E)^{n}$ with a logic topology. Let $E_{n}$ be the equivalence relation on $X^{n}$ where $\left(a_{1}, \ldots, a_{n}\right)$ and $\left(b_{1}, \ldots, b_{n}\right)$ are $E_{n}$-equivalent if $\left(a_{k}, b_{k}\right) \in E$ for all $1 \leq k \leq n$. We identify $(X / E)^{n}$ and $\left(X^{n} / E_{n}\right)$. Observe that $E_{n}$ is a $\Lambda$-definable equivalence relation on $X^{n}$. Observe that if $\mathcal{B}$ is a small collection of definable sets forming a basis for the uniform structure associated to $E$ then $\mathcal{B}_{n}$ is a small collection definable sets forming a basis for the uniform structure associated to $E_{n}$. So the uniform structure on $(X / E)^{n}$ is simply the product uniform structure.

Let $E$ be a $\bigwedge$-definable equivalence relation on $X$. A subdefinable (in $\mathcal{N}$ ) basis for $E$ is a subdefinable (in $\mathcal{N}$ ) basis for the uniform structure on $X$ associated to $E$. Lemma 4.7 is a saturation exercise which we leave to the reader.

Lemma 4.7. Suppose $\mathcal{C}$ is a small subdefinable collection of subsets of $X^{2}$. Then $\mathcal{C}$ is a subdefinable basis for $E$ if and only if

(1) $\cap \mathcal{C}=E$,

(2) each $U \in \mathcal{C}$ is symmetric, and

(3) for all $U, U^{\prime} \in \mathcal{C}$ there is $V \in \mathcal{C}$ such that $V \subseteq U \cap U^{\prime}$.

If $\mathcal{C}$ satisfies (1) and (3) then $\left\{U \cap U^{-1}: U \in \mathcal{C}\right\}$ is a subdefinable basis for $E$.

Proposition 4.8. Suppose there is a subdefinable (in $\mathcal{N}$ ) basis for $E$. Then $\mathcal{N}^{\text {Sh }}$ defines a basis for the uniform structure on $X / E$.

Proof. Lemma 4.3 shows that $E$ is externally definable and Proposition 4.4 shows that $\mathcal{N}^{\mathrm{Sh}}$ defines a basis $\mathcal{B}$ for the uniform structure on $X$. Then $\pi(\mathcal{B})$ is an $\mathcal{N}^{\mathrm{Sh}}$ definable basis for the uniform structure on $X / E$.

If the answer to Question 4.9 is positive then Proposition4.8 shows that if $\mathcal{N}$ is NIP and $E$ is externally definable then $\mathcal{N}^{\text {Sh }}$ defines a basis for the uniform structure on $X / E$.

Question 4.9. If $\mathcal{N}$ is NIP does every equivalence relation which is both $\bigwedge$-definable and externally definable admit a subdefinable basis? 
It is possible that Question 4.9 fails in general but holds under more robust modeltheoretic assumptions on $\mathcal{N}$ such as distality. At present we do not even know if Question 4.9 has a positive answer when $\mathcal{N}$ is o-minimal. Propositions 4.10 gives an answer in a very special case.

Proposition 4.10. Suppose $\mathcal{N}$ is NIP. Suppose $X$ is a definable group, $\mathcal{H}$ is a small subdefinable collection of definable subgroups of $X$, and $H:=\bigcap \mathcal{H}$. Let $E$ be equivalence modulo $H$. Then $E$ admits a subdefinable basis.

Proof. Applying the Baldwin-Saxl theorem [75, Theorem 2.13] we obtain $n$ such that for any finite $\mathcal{H}^{\prime} \subseteq \mathcal{H}$ there is $\mathcal{H}^{\prime \prime} \subseteq \mathcal{H}^{\prime}$ such that $\left|\mathcal{H}^{\prime \prime}\right| \leq n$ and $\bigcap \mathcal{H}^{\prime \prime}=\bigcap \mathcal{H}^{\prime}$. Lemma 4.7 shows that the collection of sets of the form $\left\{(g, h) \in X: g h^{-1} \in \cap \mathcal{H}^{\prime}\right\}$ for $\mathcal{H}^{\prime} \subseteq \mathcal{H},\left|\mathcal{H}^{\prime}\right| \leq n$ is a subdefinable basis for $E$.

\section{Really strong Baire CATEgory theorem}

Throughout this section $\mathcal{M}$ is a possibly multisorted first order structure, "definable" without modification means "M-definable", and $X$ is a definable set.

We prove a result on subdefinable families of nowhere dense subsets of uniform spaces in $\mathrm{NTP}_{2}$ structures. The NIP case is crucial for the proof of Theorem B.

We say that a family $\left(X_{t}\right)_{t>0}$ of sets is increasing if $X_{s} \subseteq X_{t}$ whenever $s \leq t$. The classical Baire category theorem is easily seen to be equivalent to the following: If $(Z, d)$ is a complete metric space, and $\left(X_{t}\right)_{t>0}$ is an increasing family of nowhere dense subsets of $Z$, then $\bigcup_{t>0} X_{t}$ has empty interior. It is shown in [27, Theorem D] that if $\mathcal{R}$ is a type $\mathrm{A}$ expansion of $(\mathbb{R},<,+)$ and $\left(X_{t}\right)_{t>0}$ is an increasing definable family of nowhere dense subsets of $\mathbb{R}^{n}$ then $\bigcup_{t>0} X_{t}$ is nowhere dense. This result is known as the "strong Baire category theorem". Dolich and Goodrick 17, Proposition 2.16] show that if $\mathcal{R}$ is a strong (in particular strongly dependent) expansion of a dense ordered abelian group $(R,<,+), I \subseteq R$ is an open interval, and $\left(X_{a}\right)_{a \in I}$ is an increasing definable family of discrete subsets of $R$, then $\bigcup_{a \in I} X_{a}$ is nowhere dense. A family $\mathcal{A}$ of sets is directed if for all $A, A^{\prime} \in \mathcal{A}$ there is $B \in \mathcal{A}$ such that $A \cup A^{\prime} \subseteq B$. It follows from [78, Lemma 3.5] that if $\mathcal{M}$ is a dp-minimal expansion of a divisible ordered abelian group or a non strongly minimal dp-minimal expansion of a field (equipped with the Johnson topology) and $\mathcal{A}$ is a directed definable family of nowhere dense subsets of $M^{n}$ then $\bigcup \mathcal{A}$ is nowhere dense.

Theorem 5.1 generalizes the $\mathrm{NTP}_{2}$ case of the strong Baire category theorem and the latter two results described in the preceeding paragraph.

Theorem 5.1. Suppose $\mathcal{B}$ is a subdefinable basis for a uniform structure on $X, W$ is a nonempty open subset of $X$, and $\mathcal{A}$ is a definable family of subsets of $X$ such that for every finite collection $\mathcal{U}$ of nonempty open subsets of $W$ there is $A \in \mathcal{A}$ such that $A$ is nowhere dense in $W$ and intersects each $U \in \mathcal{U}$. Then $\mathcal{M}$ is $\mathrm{TP}_{2}$.

Let $[n]$ denote $\{0, \ldots$,$\} . Let [n]^{=m}$ be the set of functions $\{0, \ldots, m\} \rightarrow\{0, \ldots, n\}$ which we identify with $\{0, \ldots, n\}$-valued sequences of length $m$. Let $[n] \leq m$ be the union of all $[n]^{=k}$ where $0 \leq k \leq m$. Given $\sigma \in[n]^{\leq m}$ and $\eta \in[n]^{\leq k}$ we let $|\sigma|$ be the length of $\sigma$, and let $\sigma^{\frown} \eta \in[n]^{\leq m+k}$ be the usual concatenation of $\sigma$ and $\eta$. 
ERIK WALSBERG

Proof. Let $R$ be the relation on $W \times(\mathcal{A} \times \mathcal{B} \times \mathcal{B})$ where $\left(a,\left(A, U, U^{\prime}\right)\right) \in R$ if and only if $a \in A[U] \backslash A\left[U^{\prime}\right]$. We show that $R$ has $\mathrm{TP}_{2}$. Fixing $n \geq 1$ we construct elements $\left(A_{i}: i \in[n]\right)$ of $\mathcal{A}$, elements $\left(U_{j}^{i}: i, j \in[n]\right)$ of $\mathcal{B}$, and nonempty open subsets $\left(W_{\sigma}: \sigma \in[n-1]^{\leq n}\right)$ of $W$ such that:

(1) $U_{j+1}^{i} \subsetneq U_{j}^{i}$ and $A_{i}\left[U_{j}^{i}\right] \backslash A_{i}\left[U_{j+1}^{i}\right]$ has interior for all $i \in[n]$ and $j \in[n-1]$,

(2) $W_{\sigma \frown i}, W_{\sigma \frown j}$ are pairwise disjoint subsets of $W_{\sigma}$ for all $\sigma \in[n-1]^{<n}$ and $i, j \in[n-1]$

(3) and if $p \in W_{\sigma}$ then $p \in A_{i}\left[U_{j}^{i}\right] \backslash A_{i}\left[U_{j+1}^{i}\right]$ if and only if $\sigma(i)=j$.

This shows that $R$ has $\mathrm{TP}_{2}$.

Let $A_{0} \in \mathcal{A}$ be nowhere dense in $W$. Applying Lemma 4.2 inductively let $U_{0}^{0}, \ldots, U_{n}^{0}$ be elements of $\mathcal{B}$ such that $U_{j+1}^{0} \subsetneq U_{j}^{0}$ and $A_{0}\left[U_{j}^{0}\right] \backslash A_{0}\left[U_{j+1}^{0}\right]$ has interior in $W$ for all $j \in[n-1]$. For all $j \in[n-1]$ let $W_{j}$ be a nonempty open subset of $W$ contained in $A_{0}\left[U_{j}^{0}\right] \backslash A_{0}\left[U_{j+1}^{0}\right]$.

Fix $1 \leq k \leq n-1$. Suppose we have constructed $\left(A_{i}: i \in[k]\right),\left(U_{j}^{i}: i \in\right.$ $[k], j \in[n])$, and $\left(W_{\sigma}: \sigma \in[n-1]^{\leq k}\right)$. Let $A_{k+1}$ be an element of $\mathcal{A}$ which is nowhere dense in $W$ and intersects every element of $\left(W_{\sigma}: \sigma \in[n-1]^{=k}\right)$. Applying Lemma 4.2 inductively let $U_{0}^{k+1}, \ldots, U_{n}^{k+1} \in \mathcal{B}$ be such that $U_{j+1}^{k+1} \subsetneq U_{j}^{k+1}$ and $A_{k+1}\left[U_{j}^{k+1}\right] \backslash A_{k+1}\left[U_{j+1}^{k+1}\right]$ has interior in each element of $\left(W_{\sigma}: \sigma \in[n-1]^{=k}\right)$ for all $j \in[n]$. For each $\sigma \in[n-1]^{=k}$ and $j \in[n-1]$ we let $W_{\sigma}{ }_{j}$ be a nonempty open subset of $W_{\sigma}$ contained in $A_{k+1}\left[U_{j}^{i}\right] \backslash A_{k+1}\left[U_{j+1}^{i}\right]$.

The statement of Proposition 5.2 is closer to previous forms of strong Baire category.

Proposition 5.2. Suppose $\mathcal{M}$ is $\mathrm{NTP}_{2}$. Suppose $\mathcal{B}$ is a subdefinable basis for a uniform structure on $X$. Let $Y$ be a definable subset of $X, \mathcal{A}$ be a subdefinable directed family of subsets of $X$, and $W$ be a nonempty open subset of $Y$. If each element of $\mathcal{A}$ is nowhere dense in $W$ then $\bigcup \mathcal{A}$ is nowhere dense in $W$.

Proof. We only treat the case when $X=Y$. The more general case follows by replacing $X$ with $Y$ and $\mathcal{B}$ with $\left.\mathcal{B}\right|_{Y}$. Suppose $\bigcup \mathcal{A}$ is somewhere dense in $W$. Let $V$ be a nonempty open subset of $Y$ in which $\bigcup \mathcal{A}$ is dense. Suppose $\mathcal{U}$ is a finite collection of nonempty open subsets of $V$. Pick for each $U \in \mathcal{U}$ an $A_{U} \in \mathcal{A}$ such that $U \cap A_{U} \neq \emptyset$. As $\mathcal{A}$ is directed there is $A \in \mathcal{A}$ such that $A_{U} \subseteq A$ for all $U \in \mathcal{U}$. This $A$ intersects each element of $\mathcal{U}$. An application of Theorem [5.1] shows that some element of $\mathcal{A}$ is somewhere dense in $W$.

Corollary 5.3 follows immediately from Proposition 5.2 .

Corollary 5.3. Suppose $\mathcal{M}$ is $\mathrm{NTP}_{2}$. Suppose $\mathcal{B}$ is a subdefinable basis for a uniform structure on $X$ with no isolated points. Let $\mathcal{A}$ be a subdefinable directed family of discrete subsets of $X$. Then $\bigcup \mathcal{A}$ is nowhere dense. In particular if $\mathcal{A}$ is a subdefinable directed family of finite subsets of $X$ then $\bigcup \mathcal{A}$ is nowhere dense.

We give three applications of these results before proving Theorem B.

5.1. Shelah expansions. Proposition 5.4 naturally goes through in greater generality, we leave that to the reader. 
Proposition 5.4. Suppose $\mathcal{M}$ is NIP and one-sorted. Suppose there is a subdefinable basis for a uniform structure on $M$. If $\mathcal{M}$ is (strongly) noiseless then $\mathcal{M}^{\mathrm{Sh}}$ is (strongly) noiseless.

Proof. We suppose that $\mathcal{M}$ is strongly noiseless and show that $\mathcal{M}^{\text {Sh }}$ is strongly noiseless, the same argument shows that if $\mathcal{M}$ is noiseless then $\mathcal{M}^{\text {Sh }}$ is noiseless. Applying Proposition 4.4 we suppose that $\mathcal{B}$ is an $\mathcal{M}^{\mathrm{Sh}}$-definable basis for the uniform structure on $M$. Suppose $X, Y$ are $\mathcal{M}^{\text {Sh }}$-definable subsets of $M^{n}$ such that $X$ is somewhere dense in $Y$ and $X$ has empty interior in $Y$. Let $U$ be a nonempty open subset of $Y$ in which $X$ is dense. As $\mathcal{B}$ is an $\mathcal{M}$-definable basis we may suppose $U$ is $\mathcal{M}^{\mathrm{Sh}}$-definable. After replacing $Y$ with $U \cap Y$ we suppose $X$ is dense in $Y$. We apply Theorem 5.1 to $Y$ and $\left.\mathcal{B}^{n}\right|_{Y}$. Applying Facts 3.2 and 3.3 we let $\left(X_{a}\right)_{a \in M^{m}}$ be an $\mathcal{M}$-definable family of sets such that for every finite $A \subseteq X$ there is $a \in M^{m}$ such that $A \subseteq X_{a} \subseteq X$. As $X$ has empty interior in $Y$ and $\mathcal{M}$ is strongly noiseless $X_{a}$ is nowhere dense in $Y$ when $X_{a} \subseteq X$. Let $\mathcal{U}$ be a finite collection of nonempty open subsets of $Y$. As $X$ is dense in $Y$ there is a finite subset $A$ of $X$ which intersects each $U \in \mathcal{U}$. So there is $a \in M^{m}$ such that $A \subseteq X_{a} \subseteq X$, this $X_{a}$ intersects each $U \in \mathcal{U}$ and is nowhere dense in $Y$. This contradicts Theorem 5.1

5.2. Cyclic orders on $(\mathbb{Z},+)$. We equip $\mathbb{R} / \mathbb{Z}$ with the cyclic group order $C$ where whenever $0 \leq a, b, c<1$ then $C(a+\mathbb{Z}, b+\mathbb{Z}, c+\mathbb{Z})$ holds if and only if either $a<$ $b<c, b<c<a$, or $c<a<b$. Given an injective character $\chi:(\mathbb{Z},+) \rightarrow(\mathbb{R} / \mathbb{Z},+)$ we let $C_{\chi}$ be pullback of $C$ by $\chi$. Then $C_{\chi}$ is a cyclic group order on $(\mathbb{Z},+)$. Tran and Walsberg show that $\left(\mathbb{Z},+, C_{\chi}\right)$ is NIP 81 .

Proposition 5.5. Let $\chi:(\mathbb{Z},+) \rightarrow(\mathbb{R} / \mathbb{Z},+)$ be an injective character. Then $\left(\mathbb{Z},+, C_{\chi},<\right)$ is $\mathrm{TP}_{2}$.

Proof. Observe that the collection of sets of the form $\{b \in \mathbb{Z}: C(a, b, c)\}, a, c \in \mathbb{Z}$ forms a $\left(\mathbb{Z},+, C_{\chi}\right)$-definable basis for a topology on $\mathbb{Z}$ with no isolated points. It follows that $\left(\mathbb{Z},+, C_{\chi}\right)$ defines a basis for a uniform structure on $\mathbb{Z}$ with no isolated points. Furthermore $\{[-n, n]: n \in \mathbb{N}\}$ is a $\left(\mathbb{Z},+, C_{\chi},<\right)$-definable directed family of finite sets with union $\mathbb{Z}$. Corollary $\left[5.3\right.$ shows that $\left(\mathbb{Z},+, C_{\chi},<\right)$ is $\mathrm{TP}_{2}$.

It is natural to ask if $\left(\mathbb{Z},+, C_{\chi},<\right)$ is totally wild. Every injective character $\chi:(\mathbb{Z},+) \rightarrow(\mathbb{R} / \mathbb{Z},+)$ is of the form $\chi(k)=\alpha k+\mathbb{Z}$ for some $\alpha \in \mathbb{R} \backslash \mathbb{Q}$. It is easy to see that $C_{\chi}$ is definable in $(\mathbb{R},<,+, \mathbb{Z}, \alpha \mathbb{Z})$. If $\alpha$ is a quadratic irrational then $(\mathbb{R},<,+, \mathbb{Z}, \alpha \mathbb{Z})$ is parameter-free intepretable in $(\mathcal{P}(\mathbb{N}), \mathbb{N}, \in, s)$ by Hieronymi 38 and hence decidable. In particular $\left(\mathbb{Z},+, C_{\chi},<\right)$ does not define multiplication on $\mathbb{Z}$.

The next result shows in fact that an $\mathrm{NTP}_{2}$ expansion of $(\mathbb{Z},<)$ cannot interpret $\left(\mathbb{Z},+, C_{\chi}\right)$. We will return to Proposition $[5.6$ around Conjecture 6 below.

Proposition 5.6. Suppose $\mathcal{Z}$ is an $\mathrm{NTP}_{2}$ expansion of $(\mathbb{Z},<)$. Let $\mathcal{G}$ be a first order expansion of a group $G$ which admits a subdefinable basis for a non-discrete group topology on $G$. Then $z$ does not interpret $\mathcal{G}$.

Proposition 5.6 fails for elementary extensions of $(\mathbb{Z},<)$. Suppose $(Z,<,+)$ is a proper elementary extension of $(\mathbb{Z},<,+)$. As $\mathbb{Z}$ is a convex subset of $Z$ an application of Fact 3.1 shows that $(Z,<,+, \mathbb{Z})$ is NIP. We declare $a+\mathbb{Z} \triangleleft b+\mathbb{Z}$ if every element of $a+\mathbb{Z}$ is strictly less then every element of $b+\mathbb{Z}$. Then $\triangleleft$ is a dense group ordering on $Z / \mathbb{Z}$. 
Proof. Suppose towards a contradiction that $z$ interprets $\mathcal{G}$. It is easy to see that any expansion of $(\mathbb{Z},<)$ eliminates imaginaries (reduce to the case when every equivalence class intersects $\mathbb{N}^{m}$, than select the lexicographic-minimal element of the intersection of each class with $\left.\mathbb{N}^{m}\right)$. So we may suppose that $\mathcal{G}$ is definable in z. Suppose $G$ is a subset of $\mathbb{Z}^{m}$. Then $\left([-n, n]^{m} \cap G\right)_{n>0}$ is a directed Z-definable family of finite subsets with union $G$. As $\mathcal{G}$ admits a subdefinable basis for a nondiscrete group topology, it admits a subdefinable basis for a uniform structure with no isolated points. So an application of Corollary 5.3 shows that $z$ is $\mathrm{TP}_{2}$.

5.3. Porosity. We give an application to the study of metric properties of definable sets in expansions of ordered fields. Porosity (for subsets of $\mathbb{R}^{n}$ ) is extensively studied in metric geometry. We discuss the natural generalization of this notion to subsets of $R^{n}, R$ an arbitrary ordered field. There are several notions of porosity in the literature, we discuss the strongest notion we are aware of.

Let $(R,<,+, \times)$ be an ordered field and $R_{>0}$ be the set of positive elements of $R$. We equip $R^{n}$ with the natural $l_{\infty} R$-valued metric. We let $B_{n}(p, r)$ be the open ball with center $p \in R^{n}$ and radius $r \in R_{>0}$. Let $X$ be a subset of $R^{n}$. Given $\delta \in R_{>0}$ we say that $X$ is $\delta$-porous if for every $p \in R^{n}, r \in R_{>0}$ there is $q \in R^{n}$ such that $B_{n}(q, \delta r) \subseteq B_{n}(p, r) \backslash X$. We say that $X$ is porous if it is $\delta$-porous for some $\delta \in R_{>0}$. It is easy to see that a porous subset of $R^{n}$ is nowhere dense. A good example of a nowhere dense non-porous subset of $\mathbb{R}$ is $\left\{n^{-k}: n \geq 1\right\}$ for any $k \geq 1$. A family $\mathcal{X}$ of subsets of $R^{n}$ is uniformly porous if there is $\delta \in R_{>0}$ such that every $X \in \mathcal{X}$ is $\delta$-porous.

Suppose $\mathcal{R}$ is a first order expansion of $(\mathbb{R},<,+, \times)$. Hieronymi and Miller [40] show that if $\mathcal{R}$ does not define the set of integers, and $X$ is a closed subset of $\mathbb{R}^{n}$ definable in $\mathcal{R}$, then the topological dimension of $X$ agrees with the Assouad dimension of $X$. (See [56] for an introduction to this important metric dimension.) By Fact 10.5 below a closed subset of $\mathbb{R}^{n}$ has topological dimension $<n$ if and only if it is nowhere dense. It follows directly from the definition that the Assouad dimension of $X \subseteq \mathbb{R}^{n}$ agrees with the Assouad dimension of the closure of $X$. Luukainen [56, Theorem 5.2] shows that a subset of $\mathbb{R}^{n}$ has Assouad dimension $<n$ if and only if it is porous. So we obtain the following.

Fact 5.7. Suppose $\mathcal{R}$ is a first order expansion of $(\mathbb{R},<,+, \times)$. If $\mathcal{R}$ does not define the set of integers then every nowhere dense subset of $\mathbb{R}^{n}$ definable in $\mathcal{R}$ is porous.

We prove a strengthening of Fact 5.7 for $\mathrm{NTP}_{2}$ expansions of ordered fields. We will not need to apply Fact 5.7 .

Proposition 5.8. Let $\mathcal{R}$ be an $\mathrm{NTP}_{2}$ expansion of $(R,<,+, \times)$. Then every $\mathcal{R}$ definable family of nowhere dense subsets of $R^{n}$ is uniformly porous. In particular every nowhere dense subset of $R^{n}$ definable in $\mathcal{R}$ is porous. If $\mathcal{R}$ is an elementary extension of an expansion of an archimedean ordered field then every nowhere dense definable subset of $R^{n}$ is $q$-porous for some $q \in \mathbb{Q}_{>0}$.

Proof. The latter two claims follows easily from the first, so we only prove the first claim. Let $\mathcal{X}=\left(X_{a}\right)_{a \in R^{m}}$ be a definable family of nowhere dense subsets of $R^{n}$. We suppose towards a contradiction that $\mathcal{X}$ is not uniformly porous. Given $a \in R^{m}$, 
$p \in R^{n}$, and $r \in R_{>0}$ we declare

$$
Y_{a, p, r}:=\frac{1}{r}\left(\left[B_{n}(p, r) \cap X_{a}\right]-p\right) .
$$

Then $\mathcal{Y}:=\left\{Y_{a, p, r}:(a, p, r) \in R^{m} \times R^{n} \times R_{>0}\right\}$ is a definable family of nowhere dense subsets of $B_{n}(0,1)$. Let $\left(U_{i}\right)_{i=1}^{k}$ be a collection of nonempty open subsets of $B_{n}(0,1)$. For each $1 \leq i \leq n$ fix $q_{i} \in U_{i}$. Let $\varepsilon \in R_{>0}$ be such that $B_{n}\left(q_{i}, \varepsilon\right) \subseteq U_{i}$ for all $1 \leq i \leq k$. Fix $a \in R^{m}$ such that $X_{a}$ is not $\varepsilon$-porous. Fix $p \in R^{n}$ and $r \in R_{>0}$ such that every open ball of radius $\varepsilon r$ contained in $B_{n}(p, r)$ intersects $X_{a}$. It is easy to see that every open ball of radius $\varepsilon$ contained in $B_{n}(0,1)$ intersects $Y_{a, p, r}$. So $Y_{a, p, r}$ intersects each $B_{n}\left(q_{i}, \varepsilon\right)$ and hence intersects each element of $\mathcal{U}$. An application of Theorem 5.1 shows that $\mathcal{R}$ is $\mathrm{TP}_{2}$, contradiction.

Suppose $\mathcal{R}$ is highly saturated, let Fin be the convex hull of $\mathbb{Q}$ in $R$, let st : Fin $\rightarrow \mathbb{R}$ be the usual standard part map, and (abusing notation) let st : Fin ${ }^{n} \rightarrow \mathbb{R}^{n}$ be

$$
\operatorname{st}\left(x_{1}, \ldots, x_{n}\right)=\left(\operatorname{st}\left(x_{1}\right), \ldots, \operatorname{st}\left(x_{n}\right)\right) .
$$

Then one can show that the following are equivalent:

(1) Every nowhere dense $\mathcal{R}$-definable subset of $R^{n}$ is $q$-porous for some $q \in \mathbb{Q}_{>0}$,

(2) $\operatorname{st}(X)$ is nowhere dense in $\mathbb{R}^{n}$ for every nowhere dense $\mathcal{R}$-definable $X \subseteq$ Fin $^{n}$.

It follows that if the unit interval in $\mathcal{R}$ is compactly dominated by st then every nowhere dense $\mathcal{R}$-definable set is $q$-porous for some $q \in \mathbb{Q}_{>0}$.

It is natural to ask if every nowhere dense definable set in an NIP expansion of an ordered field is $q$-sparse for some $q \in \mathbb{Q}_{>0}$. This need not be the case. Let $\mathcal{S}$ be an o-minimal expansion of $(\mathbb{R},<,+, \times), \mathcal{M}$ be a proper elementary extension of $\mathcal{S}$, and $\mathcal{R}$ be $(\mathcal{M}, \mathbb{R})$. Note that $\mathbb{R}$ is discrete in $M$ and is not $q$-sparse for any $q \in \mathbb{Q}_{>0}$. Such structures were first studied by van den Dries and Lewenberg [87, it is shown in [34, Theorem 5.2] that $\mathcal{R}$ is NIP.

\section{Proof of Theorem B}

We now prove Theorem B.

Theorem 6.1. Suppose $\mathcal{N}$ is highly saturated and NIP. Let $E$ be a $\Lambda$-definable equivalence relation on $X$. Suppose one of the following:

(1) $E$ is externally definable and there is a subdefinable (in $\mathcal{N}^{\mathrm{Sh}}$ ) basis for the uniform structure on $X / E$, or

(2) there is a subdefinable (in $\mathcal{N}$ ) basis for $E$.

Then the structure induced on $X / E$ by $\mathcal{N}^{\mathrm{Sh}}$ is strongly noiseless.

The proof is very similar to that of Proposition 5.4.

Proof. Lemma 4.3 and Proposition 4.4 shows that (2) implies (1), so we suppose (1). Let $\mathcal{B}$ be a subdefinable basis for the uniform structure on $X / E$. Then $\mathcal{B}_{n}$ is a subdefinable basis for the product uniform structure on $(X / E)^{n}$. Let $Y, Y^{\prime}$ be $\mathcal{N}^{\text {Sh }}$-definable subsets of $(X / E)^{n}$. We suppose that $Y$ is somewhere dense in $Y^{\prime}$ and show that $Y$ has interior in $Y^{\prime}$. Note that $\left.\mathcal{B}_{n}\right|_{Y^{\prime}}$ is an $\mathcal{N}^{\mathrm{Sh}}$-subdefinable basis for the induced uniform structure on $Y^{\prime}$. As above, let $E_{n}$ be the equivalence relation on $X^{n}$ where $\left(a_{1}, \ldots, a_{n}\right)$ and $\left(b_{1}, \ldots, b_{n}\right)$ are $E_{n}$-equivalent if and only if 
$\left(a_{k}, b_{k}\right) \in E$ for all $1 \leq k \leq n$. Recall that we identify $(X / E)^{n}$ with $X^{n} / E_{n}$ and identify the product topology on $(X / E)^{n}$ with the logic topology on $X^{n} / E_{n}$.

Let $\pi$ be the quotient map $X^{n} \rightarrow(X / E)^{n}$. Suppose towards a contradiction that $W$ is a nonempty open subset of $Y^{\prime}$ such that $Y$ is dense and co-dense in $W$. Then $Z:=\pi^{-1}(Y)$ is $\mathcal{N}^{\text {Sh }}$-definable and hence externally definable by Fact 3.2 Applying Fact 3.3 we obtain a definable family $\left(Z_{b}\right)_{b \in N^{x}}$ of subsets of $X$ such that for any finite $A \subseteq Z$ there is $b \in N^{x}$ such that $A \subseteq Z_{b} \subseteq Z$. Let $Y_{b}:=\pi\left(Z_{b}\right)$ for $b \in N^{x}$. Then $\left(Y_{b}\right)_{b \in N^{x}}$ is an $\mathcal{N}^{S h}$-definable family of sets. For any finite $A \subseteq Y$ there is $b \in N^{x}$ such that $A \subseteq Y_{b} \subseteq Z$. Suppose $\mathcal{U}$ is a finite collection of nonempty open subsets of $W$. As $Y$ is dense in $W$ there is a finite subset of $Y$ which intersects each $U \in \mathcal{U}$. So there is $b \in N^{x}$ such that $Y_{b} \subseteq Y$ and $Y_{b}$ intersects each element of $\mathcal{U}$. Proposition 4.6 shows that each $Y_{b}$ is closed in $(X / E)^{n}$. If $Y_{b} \subseteq Y$ then $Y_{b}$ is closed and co-dense in $W$ and is therefore nowhere dense in $W$. As $\mathcal{N}^{\mathrm{Sh}}$ is NIP this gives a contradiction with Theorem 5.1

\section{LOCALLY COMPACT HAUSDORFF UNIFORM STRUCTURES}

In this section we prove a more general version of Theorem A. Throughout this section, $\mathcal{M}$ is a structure, $X$ is a definable set, $\mathcal{B}$ is a subdefinable basis for a locally compact Hausdorff uniform structure on $X$, and "definable" without modification means "MN-definable".

A subdefinable compact exhaustion of $X$ is a subdefinable family $\mathcal{K}$ of compact subsets of $X$ such that every compact subset of $X$ is contained in some element of $\mathcal{K}$. We additionally assume that $X$ admits a subdefinable compact exhaustion $\mathcal{K}$. If $X$ is compact then we take $\mathcal{K}=\{X\}$ and this additional assumption is superfluous.

We equip $X^{n}$ with the product uniform structure with the subdefinable basis $\left\{U_{n}: U \in \mathcal{B}\right\}$ defined in Section 4.1. Note that $\left\{K^{n}: K \in \mathcal{K}\right\}$ is a subdefinable compact exhaustion of $X^{n}$.

Let $\mathcal{N}$ be a highly saturated elementary extension of $\mathcal{M}$. Given an $\mathcal{M}$-definable set $Z$ we let $Z^{\prime}$ be the $\mathcal{N}$-definable set defined by any formula defining $Z$. Observe that $\mathcal{B}^{\prime}:=\left\{U^{\prime}: U \in \mathcal{B}\right\}$ is a subdefinable basis for a uniform structure on $X^{\prime}$. Let $E:=\bigcap \mathcal{B}^{\prime}$. Then $\mathcal{B}^{\prime}$ is a subdefinable basis for $E$.

We define

$$
O:=\bigcup\left\{K^{\prime}: K \in \mathcal{K}\right\} .
$$

Let $\pi$ be the quotient map $X^{\prime} \rightarrow X^{\prime} / E$. Abusing notation we also let $\pi$ denote the $\operatorname{map}\left(X^{\prime}\right)^{n} \rightarrow\left(X^{\prime} / E\right)^{n}$ given by

$$
\pi\left(x_{1}, \ldots, x_{n}\right)=\left(\pi\left(x_{1}\right), \ldots, \pi\left(x_{n}\right)\right) .
$$

Lemma 7.1 is an easy variation of Proposition 4.6. We leave the details of the proof to the reader.

Lemma 7.1. Suppose $Y$ is an $\mathcal{N}$-definable subset of $\left(X^{\prime}\right)^{n}$. Then $\pi\left(Y \cap O^{n}\right)$ is closed in $(O / E)^{n}$. 
We show that $O / E$ may be identified with $X$ and view the quotient map $O \rightarrow O / E$ as a standard part map.

Lemma 7.2. If $a \in O$ then the E-class of a contains exactly one element of $X$.

We recall a useful definition from general topology. Suppose $Z$ is a topological space. A filter base on $Z$ is a collection $\mathcal{F}$ of nonempty subsets of $Z$ such that for all $E, F \in \mathcal{F}$ there is $G \in \mathcal{F}$ such that $G \subseteq E \cap F$. A point $p \in Z$ is a cluster point of a filter base $\mathcal{F}$ if every neighbourhood of $p$ intersects every element of $\mathcal{F}$. If $Z$ is compact then every filter base has a cluster point.

Proof. If $x, y \in X$ are distinct then as $X$ is Hausdorff there is $U \in \mathcal{B}$ such that $(x, y) \notin U$. So each $E$-class contains at most one element of $X$.

Fix $a \in O$. We show that $a$ is $E$-equivalent to some element of $X$. Let $K \in \mathcal{K}$ be such that $K^{\prime}$ contains $a$. We first show that $\left(U_{a}^{\prime} \cap K\right)_{U \in \mathcal{B}}$ is a filter base on $K$ and then show that the cluster point is $E$-equivalent to $a$.

Fix $U \in \mathcal{B}$. As $K$ is compact there is a finite $B \subseteq K$ such that $\left(U_{b}\right)_{b \in B}$ covers $K$. It follows that $\left(U_{b}^{\prime}\right)_{b \in B}$ covers $K^{\prime}$. So there is $b \in B$ such that $a \in U_{b}^{\prime}$. As $U^{\prime}$ is symmetric we have $b \in U_{a}^{\prime}$. So $U_{a}^{\prime} \cap K$ is nonempty for every $U \in \mathcal{B}$. For any $U, V \in \mathcal{B}$ there is $W \in \mathcal{B}$ such that $W \subseteq U \cap V$ so $W_{a}^{\prime} \subseteq U_{a}^{\prime} \cap V_{a}^{\prime}$ and hence

$$
W_{a}^{\prime} \cap K \subseteq\left(U_{a}^{\prime} \cap K\right) \cap\left(V_{a}^{\prime} \cap K\right) .
$$

So $\left(U_{a}^{\prime} \cap K\right)_{U \in \mathcal{B}}$ is a filter base on $K$. As $K$ is compact there is a cluster point $p \in K$ of $\left(U_{a}^{\prime} \cap K\right)_{U \in \mathcal{B}}$. We show that $a$ and $p$ are $E$-equivalent. It suffices to fix $U \in \mathcal{B}$ and show that $(a, p) \in U^{\prime}$. Let $V \in \mathcal{B}$ be such that $V \circ V=U$. Then $V_{p}$ intersects $V_{a}^{\prime} \cap K$. Suppose $q$ lies in this intersection. Then $(p, q) \in V^{\prime}$ and $(q, a) \in V^{\prime}$, so $(p, q) \in U^{\prime}$.

Let $\rho$ be the bijection $O / E \rightarrow X$ which maps each $E$-class to the unique element of $X$ that it contains. We consider $O / E$ as a uniform structure with basis $\pi\left(\left.\mathcal{B}^{\prime}\right|_{O}\right)$.

Lemma 7.3. Suppose $U \in \mathcal{B}$. Then $a, b \in O / E$ satisfy $(a, b) \in \pi\left(U^{\prime}\right)$ if and only if $(\rho(a), \rho(b)) \in U$. So $O / E$ and $X$ are bi-uniformly equivalent and hence homeomorphic.

Proof. Fix $a, b \in O / E$. Then $\pi(\rho(a))=a$ and $\pi(\rho(b))=b$. Recall that $(a, b) \in$ $\pi\left(U^{\prime}\right)$ if and only if $(c, d) \in U^{\prime}$ for any $c, d \in X^{\prime}$ such that $\pi(c)=a$ and $\pi(d)=$ $b$. So $(a, b) \in \pi\left(U^{\prime}\right)$ if and only if $(\rho(a), \rho(b)) \in U^{\prime}$, which holds if and only if $(\rho(a), \rho(b)) \in U$.

In light of Lemma 7.3 we identify $X$ with $O / E$ and identify the uniform structure on $X$ with that on $O / E$. We now view $\pi: O \rightarrow X$ as a standard part map. These identifications greatly simplify notation in the proof of Theorem 7.5 .

So far we have not needed to suppose that $\mathcal{B}$ is a subdefinable basis, only that each element of $\mathcal{B}$ is definable. We will now use the full assumption. As $\mathcal{B}^{\prime}$ is subdefinable basis for $E$, Lemma 4.3 shows that $E$ is externally definable in $\mathcal{N}$. Lemma 7.4 shows that $O$ is also externally definable in $\mathcal{N}$ so we regard $X$ as an imaginary sort of $\mathcal{N}^{\text {Sh }}$.

Lemma 7.4. The set $O$ is externally definable in $\mathcal{N}$. 
Proof. Let $\phi(x, y)$ be a formula such that for every $K \in \mathcal{K}$ there is $a \in M^{x}$ such that $\phi\left(a, M^{y}\right)=K$. For each $K \in \mathcal{K}$ select an element $a_{K}$ of $M^{x}$ such that $\phi\left(a_{K}, M^{y}\right)=K$. It suffices to show that the partial $x$-type

$$
p(x):=\{\neg \phi(x, b): b \in X \backslash O\} \cup\left\{\forall y\left[\phi\left(a_{K}, y\right) \rightarrow \phi(x, y)\right]: K \in \mathcal{K}\right\}
$$

is consistent. Suppose $\mathcal{C}$ is a finite subcollection of $\mathcal{K}$. Then $\bigcup \mathcal{C}$ is compact. As $\mathcal{K}$ is a subdefinable compact exhaustion of $X$ there is an $F \in \mathcal{K}$ containing $\bigcup \mathcal{C}$. Then

$$
\mathcal{N} \models\left\{\neg \phi\left(a_{F}, b\right): b \in X \backslash O\right\} \cup\left\{\forall y\left[\phi\left(a_{K}, y\right) \rightarrow \phi\left(a_{F}, y\right): K \in \mathcal{C}\right\} .\right.
$$

So $p(x)$ is consistent.

We let $\mathrm{Cl}(Y)$ be the closure in $X^{n}$ of $Y \subseteq X^{n}$. It is not a priori clear that $\mathrm{Cl}(Y)$ is always definable when $Y$ is definable, Proposition 4.4 shows that $\mathrm{Cl}(Y)$ is definable in $\mathcal{M}^{\mathrm{Sh}}$ when $\mathcal{M}$ is NIP. We let $\mathcal{X}$ be the structure induced on $X$ by $\mathcal{M}$ and as usual let $X^{\circ}$ be the open core of the structure induced on $X$ by $\mathcal{M}$, i.e. the structure on $X$ whose primitive $n$-ary relations are all sets of the form $\mathrm{Cl}(Y)$ for definable $Y \subseteq X^{n}$.

Theorem 7.5. Suppose $\mathcal{M}$ is NIP. Then $X^{\circ}$ is strongly noiseless.

Proof. Theorem 6.1 shows that the structure induced on $X^{\prime} / E$ by $\mathcal{N}^{\text {Sh }}$ is strongly noiseless. As we identify $X$ with $O / E \subseteq X^{\prime} / E$ it follows that the structure induced on $X$ by $\mathcal{N}^{\text {Sh }}$ is strongly noiseless. It now show suffices to show that $X^{\circ}$ is a reduct of the structure induced on $X$ by $\mathcal{N}^{\mathrm{Sh}}$.

We fix an $\mathcal{M}$-definable subset $Y$ of $X^{n}$ and show that $\mathrm{Cl}(Y)$ is definable in $\mathcal{N}^{\mathrm{Sh}}$. We show that $\mathrm{Cl}(Y)=\pi\left(Y^{\prime} \cap O^{n}\right)$. As $\pi$ is the identity on $X^{n}$ we have $Y \subseteq \pi\left(Y^{\prime} \cap O^{n}\right)$. Lemma 7.1 shows that $\pi\left(Y^{\prime} \cap O^{n}\right)$ is closed so $\mathrm{Cl}(Y) \subseteq \pi\left(Y^{\prime} \cap O^{n}\right)$. We prove the other inclusion. Fix $p \in \pi\left(Y^{\prime} \cap O^{n}\right)$. We show that $U_{p} \cap Y \neq \emptyset$ for all $U \in \mathcal{B}_{n}$. Fix $U \in \mathcal{B}_{n}$. Let $q \in Y^{\prime} \cap O^{n}$ satisfy $\pi(q)=p$. Then $q \in U_{p}^{\prime}$. So $Y^{\prime} \cap U_{p}^{\prime}$ is nonempty. Then $Y \cap U_{p}$ is nonempty as $\mathcal{M}$ is an elementary submodel of $\mathcal{N}$.

We will see below that strong noiselessness has very strong consequences on definable sets and functions in expansion of $(\mathbb{R},<,+)$. It is not clear how strong the assumption of strong noiselessness is in more general setttings. As an application of Theorem 7.5 we show that $X^{\circ}$-definable functions are generically continuous.

Proposition 7.6. Suppose $\mathcal{M}$ is NIP. Suppose $Y \subseteq X^{m}$ and $f: Y \rightarrow X^{n}$ are definable in $X^{\circ}$. Then there is a dense definable open subset of $Y$ on which $f$ is continuous.

Proof. Let

$$
f(a)=\left(f_{1}(a), \ldots, f_{n}(a)\right) \text { for all } a \in Y .
$$

Suppose that for all $1 \leq k \leq n$ there is a dense definable open subset $U_{k}$ of $Y$ on which $f_{k}$ is continuous. Then $\bigcap_{k=1}^{n} U_{k}$ is a dense definable open subset of $Y$ on which $f$ is continuous. So we assume $n=1$.

After applying Proposition 4.4 and replacing $\mathcal{M}$ by $\mathcal{M}^{\mathrm{Sh}}$ we may suppose that $\mathcal{B}$ is a definable basis.

For each $U \in \mathcal{B}$ we let $D_{U}$ be the set of $a \in Y$ such that for all $V \in \mathcal{B}$ there is $b \in Y$ such that $(a, b) \in V$ and $(f(a), f(b)) \notin U$. Note that $\left(D_{U}\right)_{U \in \mathcal{B}}$ is a definable 
family and $\bigcup_{U \in \mathcal{B}} D_{U}$ is the set of points at which $f$ is discontinuous. We suppose towards a contradiction that $\bigcup_{U \in \mathcal{B}} D_{U}$ is somewhere dense in $Y$. Observe that if $U, V \in \mathcal{B}$ satisfy $U \subseteq V$ then $D_{V} \subseteq D_{U}$. For any $U, U^{\prime} \in \mathcal{B}$ there is $V \in \mathcal{B}$ such that $V \subseteq U \cap U^{\prime}$, hence $D_{U}, D_{U^{\prime}} \subseteq D_{V}$. So $\left(D_{U}\right)_{U \in \mathcal{B}}$ is directed. We apply Proposition 5.2 to obtain $U \in \mathcal{B}$ such that $D_{U}$ is somewhere dense in $Y$. It follows by strong noiselessness that $D_{U}$ has nonempty interior in $Y$. Let $W$ be a nonempty open subset of $Y$ contained in $D_{U}$. As $\mathcal{B}$ is a definable basis we may suppose $W$ is definable.

For each $K \in \mathcal{K}$ let $F_{K}$ be $f^{-1}(K) \cap D_{U}$. Then $\left(F_{K}\right)_{K \in \mathcal{K}}$ is subdefinable. For any $K, L \in \mathcal{K}$ there is $P \in \mathcal{K}$ such that $K, L \subseteq P$, hence $F_{K}, F_{L} \subseteq F_{P}$. So $\left(F_{K}\right)_{K \in \mathcal{K}}$ is directed. As $\bigcup_{K \in \mathcal{K}} F_{K}=D_{U}$ Proposition 5.2 and strong noiselessness yield a $K \in \mathcal{K}$ such that $F_{K}$ has interior in $W$. After replacing $W$ with a smaller nonempty definable open set if necessary we suppose that $W$ is contained in $F_{K}$. So $f(W) \subseteq K$.

Fix $V \in \mathcal{B}$ such that $V \circ V \subseteq U$. As $K$ is compact there is a finite $A \subseteq K$ such that $\left(V_{a}\right)_{a \in A}$ covers $K$. Then $\left(f^{-1}\left(V_{a}\right)\right)_{a \in A}$ covers $F_{K}$ and in particular covers $W$. So there is $b \in A$ such that $f^{-1}\left(V_{b}\right)$ is somewhere dense in $W$. Then $f^{-1}\left(V_{b}\right)$ has interior in $W$ by strong noiselessness. Let $Z$ be the interior of $f^{-1}\left(V_{b}\right)$ in $W$,

note $Z$ is definable as $\mathcal{B}$ is a definable basis. As $p \in D_{U}$ there is $q \in Z$ such that $(f(p), f(q)) \notin U$. However as $p, q \in f^{-1}\left(V_{b}\right)$ we have $(f(p), b) \in V$ and $(b, f(q)) \in V$, so $(f(p), f(q)) \in U$ by choice of $V$. Contradiction.

\section{Two COUNTEREXAMPLES}

It is natural to ask if Theorem 7.5 holds when we only have a definable basis for a locally compact Hausdorff topology on $X$. We show that this is not the case. In both 8.1 and 8.2 we let $I$ be $[0,1]$.

8.1. The Cantor function. Let $K$ be the middle-thirds Cantor set and $f: I \rightarrow I$ be the Cantor function. If $t \in K$ and $t=\sum_{i=1}^{\infty} a_{i} 3^{-i}$ where $a_{i} \in\{0,2\}$ for all $i$, then $f(t)=\sum_{i=1}^{\infty} \frac{1}{2} a_{i} 2^{-i}$ and if $t \notin K$ then $f(t)=\sup \{f(s): s \in K, s<t\}$. See [20] for background. Continuity of $f$ implies $(\mathbb{R},<, f)$ and $(\mathbb{R},<, f)^{\circ}$ are interdefinable.

We first show that $(\mathbb{R},<, f)$ is NIP (in fact dp-minimal). A subset $X$ of $\mathbb{R}^{2}$ is monotone if whenever $\left(s, s^{\prime}\right) \in X$ and $t \leq s, s^{\prime} \leq t^{\prime}$ then $\left(t, t^{\prime}\right) \in X$. It is a special case of 74, Proposition 4.2] that the expansion of $(\mathbb{R},<)$ by all monotone subsets of $\mathbb{R}^{2}$ is dp-minimal. Suppose $h: \mathbb{R} \rightarrow \mathbb{R}$ is increasing. Then $G_{h}:=\left\{\left(s, s^{\prime}\right) \in \mathbb{R}^{2}: g(s) \leq s^{\prime}\right\}$ is monotone. It is easy to see that $h$ is definable in $\left(\mathbb{R},<, G_{h}\right)$. It follows that the expansion of $(\mathbb{R},<)$ by all increasing functions $\mathbb{R} \rightarrow \mathbb{R}$ is dp-minimal. As $f(0)=0$ and $f(1)=1$, we let $h: \mathbb{R} \rightarrow \mathbb{R}$ be given by $h(t)=0$ when $t<0, h(t)=f(t)$ when $t \in I$, and $h(t)=1$ when $t>1$. Then $h$ is increasing and $(\mathbb{R},<, h)$ is interdefinable with $(\mathbb{R},<, f)$. So $(\mathbb{R},<, f)$ is dp-minimal.

It follows from the definition of $f$ that $K$ is the set of $t \in[0,1]$ at which $f$ is not locally constant. So $K$ is definable in $(\mathbb{R},<, f)$. Each connected component of $I \backslash K$ is an open interval, let $L$ be the set of endpoints of connected components of $I \backslash K$. Note that $L$ is definable in $(\mathbb{R},<, f)$. As $f(K)=I, L$ is dense in $K$, and $f$ is 
continuous, we see that $f(L)$ is dense in $I$. As $L$ is countable $f(L)$ is co-dense in I. So $(\mathbb{R},<, f)$ is noisey.

8.2. The double arrow space. Our second counterexample is definable in $(\mathbb{R},<)$. Let $X$ be $I \times\{0,1\}$. Let $\triangleleft$ be the lexicographic order on $X$, i.e. $(s, t) \triangleleft\left(s^{\prime}, t^{\prime}\right)$ if either $s<s^{\prime}$ or $s=s^{\prime}$ and $t<t^{\prime}$. We equip $X$ with the associated order topology, this is known as the double arrow space or the split interval. This topology is compact Hausdorff as $\triangleleft$ is complete and has a maximum and minimum. Any linear order is dp-minimal [74, Proposition 4.2], so $(X, \triangleleft)$ is dp-minimal.

Note that the collection of $\triangleleft$-open intervals is a $(X, \triangleleft)$-definable basis for the double arrow space. Note that $\triangleleft$ is open in the product topology on $X^{2}$, so $(X, \triangleleft)$ and $(X, \triangleleft)^{\circ}$ are interdefinable. Now $I \times\{0\}$ is the set of $a \in X$ such that $\{b \in X: a \triangleleft b\}$ has a minimum. (If $a=(t, 0)$ then this minimum is $(t, 1)$.) So $I \times\{0\}$ and $I \times\{1\}$ are both definable in $(X, \triangleleft)$. Finally $I \times\{0\}$ and $I \times\{1\}$ are both dense in $X$. So $(X, \triangleleft)$ is noisey.

A similar argument shows that $(K,<)$ is dp-minimal, interdefinable with $(K,<)^{\circ}$, and noisey, when $K$ is any Cantor subset of $\mathbb{R}$.

\section{EXPANSIONS OF LOCALLY COMPACT GROUPS}

Let $G$ be a group. Suppose that $\mathcal{G}$ is a first order expansion of $G, \mathcal{C}$ is a subdefinable neighbourhood basis at the identity for a locally compact Hausdorff group topology on $G$, and suppose $\mathcal{K}$ is a subdefinable compact exhaustion of $G$. If $G$ is compact then we take $\mathcal{K}=\{G\}$.

Recall that the collection $\left\{\left(g, g^{\prime}\right) \in G^{2}: g^{-1} g^{\prime} \in U\right\}, U \in \mathcal{C}$ is a subdefinable basis for a uniform structure on $G$ inducing the group topology. Theorem 9.1 is a special case of Theorem 7.5 .

Theorem 9.1. Suppose $\mathcal{G}$ is NIP. Then $\mathcal{G}^{\circ}$ is strongly noiseless.

Theorem 9.1 shows that any $\mathcal{G}^{\circ}$-definable subset of $G^{n}$ with empty interior is topologically small. Under the additional assumption that $G$ is second countable we show that any $\mathcal{G}^{\circ}$-definable subset of $G^{n}$ with empty interior is measure-theoretically small. The key tool is a theorem of Simon [76]. Proposition 9.2 is already known for expansions of $(\mathbb{R},<,+)$. By [27, Theorem $\mathrm{D}]$ any nowhere dense subset of $\mathbb{R}^{n}$ definable in a type $\mathrm{A}$ expansion of $(\mathbb{R},<,+)$ is Lebesgue null.

Proposition 9.2. Suppose $G$ is second countable and $\mathcal{G}$ is NIP. Then any $\mathcal{G}^{\circ}$ definable subset of $G^{n}$ with empty interior is Haar null.

The Haar measure is unique up to rescaling, so the collection of Haar null sets does not depend on the choice of a Haar measure. An open subset of a locally compact second countable group has non-zero Haar measure, so we see that a subset of $G^{n}$ definable in $\mathcal{G}^{\circ}$ is Haar null if and only if it has empty interior.

Proof. Let $X$ be an $\mathcal{G}^{\circ}$-definable subset of $G^{n}$ with empty interior and let $\mathrm{Cl}(X)$ be the closure of $X$ in $G^{n}$. Then $X$ is nowhere dense, so $\mathrm{Cl}(X)$ is nowhere dense. Corollary 4.5 shows that $\mathrm{Cl}(X)$ is $\mathcal{G}^{\text {Sh }}$-definable. As $\mathcal{G}^{\text {Sh }}$ is NIP [76, Theorem 3.6] shows that $\mathrm{Cl}(X)$ is Haar null. So $X$ is Haar null. 
We finish this section with two examples.

Suppose $\mathcal{R}$ is a first order expansion of $(\mathbb{R},<,+)$. Then $\{(-t, t): t>0\}$ is a definable neighbourhood basis of zero and $\{[-t, t]: t>0\}$ is a definable compact exhaustion of $\mathbb{R}$. Theorem 9.3 follows from Theorem 9.1 and Theorem 2.17

Theorem 9.3. Suppose $\mathcal{R}$ is NIP. Then $\mathcal{R}^{\circ}$ is generically locally o-minimal.

We now discuss structures on the $p$-adics. Fix a prime $p$. Let $\overline{\mathbb{Q}}_{p}$ be the field of $p$-adic numbers. Let $v_{p}$ be the $p$-adic valuation on $\mathbb{Q}_{p}$. Let $\prec$ be the binary relation on $\mathbb{Q}_{p}$ where $a \prec b$ if $v_{p}(a) \geq v(p)$. It is well-known that $\prec$ is definable in $\overline{\mathbb{Q}}_{p}$ (see for example [3, Section 2.1]) so $\left(\mathbb{Q}_{p},+, \prec\right)$ is a reduct of $\overline{\mathbb{Q}}_{p}$. Observe that the collection of sets of the form $\left\{a \in \mathbb{Q}_{p}: a \prec b\right\}, b \in \mathbb{Q}_{p}$ is both a definable neighbourhood basis at the identity and a definable compact exhaustion of $\mathbb{Q}_{p}$.

Theorem 9.4. Suppose $\mathcal{Q}$ is a first order expansion of $\left(\mathbb{Q}_{p},+, \prec\right)$. If $\mathcal{Q}$ is NIP then $\mathbf{Q}^{\circ}$ is strongly noiseless. In particular an NIP expansion of $\overline{\mathbb{Q}}_{p}$ has strongly noiseless open core.

We finish this section with two questions on expansions of $\overline{\mathbb{Q}}_{p}$. It is shown in 17 , Corollary 2.4] that if $\mathcal{R}$ is a strongly dependent expansion of $(\mathbb{R},<,+, \times)$ then $\mathcal{R}^{\circ}$ is o-minimal (see Theorem 11.9). Recall that an expansion of $(\mathbb{R},<)$ is o-minimal if and only if every definable subset of $\mathbb{R}$ is the union of an open set and a finite set.

Question 9.5. Suppose $\mathcal{Q}$ is a strongly dependent expansion of $\overline{\mathbb{Q}}_{p}$. Must every $\mathrm{Q}^{\circ}$-definable subset of $\mathbb{Q}_{p}$ be a union of an open set and a finite set?

Question 9.5 fails if we only assume $\mathcal{Q}$ is NIP. Mariaule [58] shows that $\left(\overline{\mathbb{Q}}_{p}, p^{\mathbb{Z}}\right)$ is NIP. As $p^{\mathbb{Z}}$ has closure $p^{\mathbb{Z}} \cup\{0\}$ we see that $\left(\overline{\mathbb{Q}}_{p}, p^{\mathbb{Z}}\right)$ is interdefinable with $\left(\overline{\mathbb{Q}}_{p}, p^{\mathbb{Z}}\right)^{\circ}$. If Question 9.5 admits a positive answer then one can apply Dolich and Goodrick [16, Theorem 3.18] to get a weak cell decomposition for $\mathrm{Q}^{\circ}$-definable sets.

It is shown in 43 that any function $\mathbb{R}^{m} \rightarrow \mathbb{R}^{n}$ definable in a noiseless expansion of $(\mathbb{R},<,+)$ is differentiable on a dense open subset of $\mathbb{R}^{m}$. Kuijpers and Leenknegt [53. Theorem 1.9] show that any function $\mathbb{Q}_{p} \rightarrow \mathbb{Q}_{p}$ definable in a Pminimal expansion of the field of $p$-adic numbers is differentiable away from a finite set. (Recall that P-minimal structures are NIP.)

Conjecture 3. Suppose $\mathcal{Q}$ is an NIP expansion of the field of p-adic numbers. Any function $\mathbb{Q}_{p}^{m} \rightarrow \mathbb{Q}_{p}^{n}$ definable in $Q^{\circ}$ is differentiable on a dense open subset of $\mathbb{Q}_{p}^{m}$.

Note that Proposition 7.6 shows that any function $\mathbb{Q}_{p}^{m} \rightarrow \mathbb{Q}_{p}^{n}$ definable in $Q^{\circ}$ is continuous on a dense open subset of $\mathbb{Q}_{p}^{m}$. Conjecture 3 and a positive answer to Question 9.5 would together generalize the theorem of Kuijpers and Leenknegt to continuous definable functions in strongly dependent expansions of $\overline{\mathbb{Q}}_{p}$.

\section{Generic local O-Minimality}

Throughout this section we assume $\mathcal{R}$ is a generically locally o-minimal expansion of $(\mathbb{R},<,+)$ and throughout "definable" without modification means "R-definable". By Theorem 9.3 all results in this section hold for NIP expansions of $(\mathbb{R},<,+)$ by closed subsets of Euclidean space. Conjecture 2 implies 
that the results of this section hold for any expansion of $(\mathbb{R},<,+)$ by closed subsets of Euclidean space which does not define an isomorphic copy of $(\mathcal{P}(\mathbb{N}), \mathbb{N}, \in, s)$.

We will need to recall various results from the general theory of expansions of $(\mathbb{R},<,+)$, most of which are stated in terms of $D_{\Sigma}$-sets. This collection of definable sets was introduced by Dolich, Miller, and Steinhorn 18. A subset of $\mathbb{R}^{n}$ is $D_{\Sigma}$ if there is a definable family $\left(X_{s, t}\right)_{s, t>0}$ of compact subsets of $\mathbb{R}^{n}$ such that $X_{s^{\prime}, t} \subseteq X_{s, t}$ when $s^{\prime} \leq s, X_{s, t^{\prime}} \subseteq X_{s, t}$ when $t \leq t^{\prime}$, and $X=\bigcup_{s, t>0} X_{s, t}$. Every constructible definable set is $D_{\Sigma}$ and $D_{\Sigma}$-sets are closed under finite unions, finite intersections, products, and images under continuous definable functions [18, 1.9,1.10]. (These facts hold for arbitrary expansions of $(\mathbb{R},<,+)$.)

We refer to Fact 10.1, proven in [42, Proposition 6.3], as "definable selection". Definable selection can fail for noisey NIP expansions such as $(\mathbb{R},<,+, \mathbb{Q})$ (see 42$]$ for this example).

Fact 10.1. Let $X \subseteq \mathbb{R}^{m} \times \mathbb{R}^{n}$ be $\emptyset$-definable and let $\pi: \mathbb{R}^{m} \times \mathbb{R}^{n} \rightarrow \mathbb{R}^{m}$ be the coordinate projection onto $\mathbb{R}^{m}$. Then there is a $\emptyset$-definable function $f: \pi(X) \rightarrow \mathbb{R}^{n}$ such that

(1) $(a, f(a)) \in A$ for all $a \in \pi(X)$.

(2) $f(a)=f(b)$ for all $a, b \in \pi(X)$ such that $X_{a}=X_{b}$.

So $\mathcal{R}$ admits definable Skolem functions and eliminates imaginaries.

Let $X$ be a subset of $\mathbb{R}^{n}$ and $p \in X$. We say that $p$ is a $\mathbf{C}^{\mathbf{k}}$-point of $X$ if the following holds (possibly after permuting coordinates): there is $0 \leq d \leq n$, an open subset $V$ of $\mathbb{R}^{d}$, a $C^{k}$-function $f: V \rightarrow \mathbb{R}^{n-d}$, and an open neighbourhood $U$ of $p$ such that $\operatorname{gr}(f)=U \cap X$. An application of the inverse function theorem shows that if $k \geq 1$ then $p$ is a $C^{k}$-point of $X$ if and only if there is an open neighbourhood $U$ of $p$ such that $U \cap X$ is a $C^{k}$-submanifold of $\mathbb{R}^{n}$. The $C^{k}$-points of $X$ form a $C^{k}$-submanifold of $\mathbb{R}^{n}$. It easy to see that $p$ is a $C^{0}$-point of $X$ if there is $0 \leq d \leq n$, a coordinate projection $\pi: \mathbb{R}^{n} \rightarrow \mathbb{R}^{d}$, an open neighbourhood $U$ of $p$, and an open subset $V$ of $\mathbb{R}^{d}$, such that $\pi$ induces a homeomorphism $U \cap X \rightarrow V$. So Fact 2.18 shows that if $X$ is definable then the $C^{0}$-points of $X$ are dense in $X$.

It is easy to see that the $C^{0}$-points of $X$ form an $(\mathbb{R},<,+, X)$-definable set. It is an open question whether the $C^{1}$-smooth points of $X$ always form an $(\mathbb{R},<,+, X)$ definable set. The $C^{\infty}$-smooth points of $X$ need not form an $(\mathbb{R},<,+, \times, X)$ definable set by work of Le Gal and Rolin [55]. Fact 10.2 is proven in [45].

Fact 10.2. Let $X$ be a subset of $\mathbb{R}^{n}$ and $k \geq 2$. Then the set of $C^{k}$-points of $X$ is $(\mathbb{R},<,+, X)$-definable.

Let $U$ be an open subset of $\mathbb{R}^{m}$. Miller [63, Theorem 3.3] shows that a function $U \rightarrow \mathbb{R}^{m}$ definable in a noiseless expansion is continuous on a dense definable open subset of $U$. It is shown in [43] that a continuous function $U \rightarrow \mathbb{R}^{n}$ definable in a type A expansion is $C^{k}$ on a dense definable open subset of $U$ for any $k \geq 1$. Theorem 10.3 follows.

Theorem 10.3. Suppose $U$ is a definable open subset of $\mathbb{R}^{m}$ and $f: U \rightarrow \mathbb{R}^{n}$ is a definable function. Fix $k \geq 0$. Then there is a dense definable open subset $V$ of $U$ such that the restriction of $f$ to $V$ is $C^{k}$. 
It is an open question whether a function $\mathbb{R} \rightarrow \mathbb{R}$ definable in an o-minimal expansion of $(\mathbb{R},<,+, \times)$ is $C^{\infty}$ on a dense open subset of $\mathbb{R}$. We now obtain generic $C^{k}$-smoothness for definable sets. It follows from Theorem 2.17 that Theorem 10.4 fails for expansions of $(\mathbb{R},<,+)$ which are not generically locally o-minimal, we leave the details to the reader.

Theorem 10.4. Let $X$ be a definable subset of $\mathbb{R}^{n}$. Fix $k \geq 2$. Then the $C^{k}$-points of $X$ form a dense definable subset of $X$. It follows that there is a definable open $W \subseteq \mathbb{R}^{n}$ such that $W \cap X$ is a $C^{k}$-submanifold of $\mathbb{R}^{n}$ and $W$ is dense in $X$.

Let $Y$ be the set of $C^{k}$-points of $X$. Theorem 10.4. Theorem 2.13, and the frontier inequality for o-minimal structures may be applied to show that if $\mathcal{R}$ is locally ominimal then $\operatorname{dim} X \backslash Y<\operatorname{dim} X$. This inequality fails in general when $\mathcal{R}$ is not locally o-minimal. Suppose $\mathcal{R}$ is not locally o-minimal. Applying Theorem 2.13 we obtain a definable discrete subset $D$ of $\mathbb{R}_{>0}$ such that $\operatorname{Cl}(D)=D \cup\{0\}$. Then for any $k, m \geq 0$, the set of $C^{k}$-points of $\operatorname{Cl}(D) \times \mathbb{R}^{m}$ is $D \times \mathbb{R}^{m}$ and both $D \times \mathbb{R}^{m}$ and $\{0\} \times \mathbb{R}^{m}$ are $m$-dimensional.

Proof. An application of Fact 10.2 shows that the set of $C^{k}$-points of $X$ is definable. Fix an open subset $U$ of $\mathbb{R}^{n}$ such that $U \cap X \neq \emptyset$. We show that $U$ contains a $C^{k}$-point of $X$. After applying Fact 2.18, permuting coordinates, and shrinking $U$ if necessary we obtain $0 \leq d \leq n$, a definable open $V \subseteq \mathbb{R}^{d}$, and a definable continuous $f: V \rightarrow \mathbb{R}^{n-d}$ such that $\operatorname{gr}(f)=U \cap X$. Applying Theorem 10.3 we obtain a dense definable open subset $V^{\prime}$ of $V$ such that $f$ is $C^{k}$ on $V^{\prime}$. Fix $p \in V^{\prime}$. Then $(p, f(p))$ is a $C^{k}$-point of $X$.

We now prove the second claim. Let $Y$ be the set of $C^{k}$-points of $X$. Note that $Y$ is open in $X$. Let $W$ be the union of all open boxes $B$ in $\mathbb{R}^{n}$ such that $B \cap Y \neq \emptyset$ and $B \cap X \subseteq Y$. Then $W$ is definable and $W \cap X=Y$.

We now develop a theory of dimension for definable sets. We make crucial use of the dimension theory for $D_{\Sigma}$-sets in type A expansions developed in 27 . We first recall a classical theorem of Menger and (independently) Uryshon [24, 1.8.10].

Fact 10.5. Suppose $X \subseteq \mathbb{R}^{n}$. Then $\operatorname{dim} X=n$ if and only if $X$ has interior.

Given a nonempty subset $X$ of $\mathbb{R}^{n}$ we let $\operatorname{Dim} X$ be the maximal $0 \leq d \leq n$ for which there is a coordinate projection $\pi: \mathbb{R}^{n} \rightarrow \mathbb{R}^{d}$ such that $\pi(X)$ has interior and let $\operatorname{Dim} \emptyset=-1$. Fact 10.6 holds more generally for noiseless expansions of $(\mathbb{R},<,+)$. It is proven in [63, Section 7].

Fact 10.6. Suppose $X$ is a definable subset of $\mathbb{R}^{n}$. Then $\operatorname{Dim} X=\operatorname{Dim} \operatorname{Cl}(X)$.

Fact 10.7 holds more generally for type A expansions [27, Theorem E].

Fact 10.7. Suppose $X$ is a $D_{\Sigma}$ subset of $\mathbb{R}^{n}$. Then $\operatorname{dim} X=\operatorname{Dim} X$.

Proposition 10.8 fails for noisey expansions. If $X$ is a dense and co-dense subset of a nonempty open interval $I$ then $X$ is zero-dimensional and $\mathrm{Cl}(X)=\mathrm{Cl}(I)$ is one-dimensional.

Proposition 10.8. Suppose $X$ is a definable subset of $\mathbb{R}^{n}$. Then $\operatorname{dim} X=\operatorname{dim} \operatorname{Cl}(X)$.

Proof. If $X$ is empty then both dimensions are -1 . Suppose $X \neq \emptyset$. Monotonicity of topological dimensions yields $\operatorname{dim} X \leq \operatorname{dim} \mathrm{Cl}(X)$. We prove the other inequality. 
Suppose $\operatorname{dim} \operatorname{Cl}(X)=d$. Then $\operatorname{Dim} \operatorname{Cl}(X)=d$ by Fact 10.7 and so $\operatorname{Dim} X=d$ by Fact 10.6. Let $\pi: \mathbb{R}^{n} \rightarrow \mathbb{R}^{d}$ be a coordinate projection such that $\pi(X)$ contains a nonempty open subset $U$ of $\mathbb{R}^{n}$. After permuting coordinates if necessary and applying definable selection we let $f: U \rightarrow \mathbb{R}^{n-d}$ be definable such that $\operatorname{gr}(f) \subseteq$ $X$. After applying Theorem 10.3 and shrinking $U$ if necessary we suppose $f$ is continuous. Then $\operatorname{gr}(f)$ is homeomorphic to $U$ and so has topological dimension $d$. Thus $\operatorname{dim} X \geq d$.

Corollary 10.9 follows from Proposition 10.8 and its proof.

Corollary 10.9. Suppose $X$ is a nonempty definable subset of $\mathbb{R}^{n}$. If $\operatorname{dim} X=d$ then there is a nonempty definable open $U \subseteq \mathbb{R}^{d}$ and a definable injection $f: U \rightarrow$ $X$.

We view Theorem 10.10 as a generalization of Fact 10.5 for definable sets. Theorem 10.10 fails for general closed subsets of $\mathbb{R}^{2}$. For example if $f:[0,1] \rightarrow[0,1]$ is the Cantor function, $K$ is the middle-thirds Cantor set, and $G:=\{(t, f(t)): \in[0,1]\}$, then $\operatorname{dim} G=0$ and $\operatorname{Dim} G=1$. It is an open question whether Theorem 10.10 holds for noiseless expansions of $(\mathbb{R},<,+)$.

Theorem 10.10. Suppose $X$ is a definable subset of $\mathbb{R}^{n}$. Then $\operatorname{dim} X=\operatorname{Dim} X$.

Proof. Proposition 10.8 shows that $\operatorname{dim} X=\operatorname{dim} \operatorname{Cl}(X)$. As any closed definable set is $D_{\Sigma}$ an application of Fact 10.7 shows that $\operatorname{dim} \mathrm{Cl}(X)$ and $\operatorname{Dim} \mathrm{Cl}(X)$ agree. Fact 10.6 shows $\operatorname{Dim} \operatorname{Cl}(X)=\operatorname{Dim} X$. So $\operatorname{dim} X=\operatorname{Dim} X$.

Corollary 10.11 follows easily from Theorem 10.10. Corollary 10.11 may be easily applied to extend our dimension theory to other models of the theory of $\mathcal{R}$. We leave this to the reader.

Corollary 10.11. Suppose $X$ is a definable subset of $\mathbb{R}^{m} \times \mathbb{R}^{n}$ and let $-1 \leq d \leq n$. Then $\left\{a \in \mathbb{R}^{m}: \operatorname{dim} X_{a}=d\right\}$ is definable.

Corollary 10.12 fails if $\mathcal{R}$ is noisey. If $X$ is a dense and co-dense subset of a nonempty open interval $I$ then $X, I \backslash X$ are both zero-dimensional and $I$ is one-dimensional.

Corollary 10.12. Suppose $X$ is a definable set and $\mathcal{Y}$ is a finite partition of $X$ into definable sets. Then

$$
\operatorname{dim} X=\max \{\operatorname{dim} Y: Y \in \mathcal{Y}\} .
$$

Proof. Monotonicity of topological dimension implies that $\operatorname{dim} X \geq \operatorname{dim} Y$ for all $Y \in \mathcal{Y}$. We prove the other inequality. Suppose $\operatorname{dim} X=d$. By Theorem 10.10 there is a coordinate projection $\pi: \mathbb{R}^{n} \rightarrow \mathbb{R}^{d}$ such that $\pi(X)$ has interior. As $\{\pi(Y): Y \in \mathcal{Y}\}$ covers $\pi(X)$ there is a $Y \in \mathcal{Y}$ such that $\pi(Y)$ is somewhere dense in $\mathbb{R}^{d}$. Then $\pi(Y)$ has interior as $\mathcal{R}$ is noiseless. So $\operatorname{dim} Y \geq d$ by Theorem 10.10 ,

Proposition 10.13. Let $X$ be a definable subset of $\mathbb{R}^{n}$. Fix $k \geq 0$. Then $\operatorname{dim} X$ is the maximal $d$ for which there is a definable open subset $U$ of $\mathbb{R}^{n}$ such that $U \cap X$ is a d-dimensional $C^{k}$-submanifold of $\mathbb{R}^{n}$. In particular $\operatorname{dim} X$ is the maximal $d$ for which there is a definable open subset $U$ of $\mathbb{R}^{n}$ such that $X$ is closed in $U$ and $\operatorname{dim} U \cap X=d$.

Proof. The second claim follows directly from the first. If there is an open $U \subseteq \mathbb{R}^{n}$ such that $U \cap X$ is a $d$-dimensional $C^{k}$-submanifold then $\operatorname{dim} X \geq d$ by monotonicity 
of topological dimension. We prove the other inequality. Suppose $\operatorname{dim} X=d$. Let $l=\max \{k, 2\}$. Applying Theorem 10.4 we obtain a definable open subset $U$ of $\mathbb{R}^{n}$ such that $U \cap X$ is a $C^{l}$-submanifold of $\mathbb{R}^{n}$ and $U$ is dense in $X$. As $\operatorname{Cl}(U \cap X)=X$ an application of Proposition 10.8 shows that $U \cap X$ is $d$-dimensional.

Theorem 10.14 fails for noisey expansions. If $X$ is a dense and co-dense subset of a nonempty open interval $I$ then $Y:=[\{0\} \times X] \cup[\{1\} \times(I \backslash X)]$ is zero-dimensional and the projection of $Y$ onto the second coordinate is one-dimensional.

Theorem 10.14. Let $X$ be a definable subset of $\mathbb{R}^{m}$ and suppose $f: X \rightarrow \mathbb{R}^{n}$ is definable. Then $\operatorname{dim} f(X) \leq \operatorname{dim} X$. In particular dimension is preserved by definable bijections.

Proof. The second claim follows easily from the first so we only prove the first claim. Suppose $\operatorname{dim} f(X)=d$. Theorem 10.10 yields a coordinate projection $\pi: \mathbb{R}^{n} \rightarrow \mathbb{R}^{d}$ such that $\pi(f(X))$ has interior. After replacing $f$ with $\pi \circ f$ we suppose that $f(X)$ has interior. Let $U \subseteq X$ be a definable open subset of $\mathbb{R}^{n}$. Applying definable selection we obtain a definable $g: U \rightarrow X$ such that $f(g(p))=p$ for all $p \in U$. Note that $g$ is injective. After shrinking $U$ if necessary and applying Theorem 10.3 we suppose $g$ is continuous. Let $K$ be a compact subset of $U$ with nonempty interior. Then $\operatorname{dim} K=d$. As $g$ is a continuous injection and $K$ is compact, $g$ gives a homeomorphism $K \rightarrow g(K)$. Then $\operatorname{dim} g(K)=d$ and so $\operatorname{dim} X \geq d$.

We now proceed towards the proof of Theorem 10.17 .

We recall two results on the dimension of $D_{\Sigma}$-sets in type A expansions of $(\mathbb{R},<,+)$. If $X \subseteq \mathbb{R}^{m} \times \mathbb{R}^{n}$ is $D_{\Sigma}$ then $\left\{a \in \mathbb{R}^{m}: \operatorname{dim} X_{a} \geq d\right\}$ is always $D_{\Sigma}$ [27, Fact 2.9] but $\left\{a \in \mathbb{R}^{m}: \operatorname{dim} X_{a}=d\right\}$ need not be $D_{\Sigma}$ (the complement of $D_{\Sigma}$-set may not be $D_{\Sigma}$ ). Fact 10.15 holds in any type A expansion [27, Theorem 6.4, Corollary 6.7].

Fact 10.15. Let $X$ be a $D_{\Sigma}$-subset of $\mathbb{R}^{m} \times \mathbb{R}^{n}$ and let $\pi$ be the coordinate projection $\mathbb{R}^{m} \times \mathbb{R}^{n} \rightarrow \mathbb{R}^{m}$. Then there is $0 \leq d \leq m$ such that

$$
\operatorname{dim} X \leq\left\{a \in \mathbb{R}^{m}: \operatorname{dim} X_{a} \geq d\right\}+d .
$$

If there is $a 0 \leq d \leq n$ such that $\operatorname{dim} X_{a}=d$ for all $a \in \pi(X)$ then

$$
\operatorname{dim} X=\operatorname{dim} \pi(X)+d .
$$

Theorem 10.16. Let $X$ be a definable subset of $\mathbb{R}^{m} \times \mathbb{R}^{n}$. Then

$$
\operatorname{dim} X=\max _{0 \leq d \leq n} \operatorname{dim}\left\{a \in \mathbb{R}^{m}: \operatorname{dim} X_{a}=d\right\}+d .
$$

In particular

$$
\operatorname{dim} Y \times Y^{\prime}=\operatorname{dim} Y+\operatorname{dim} Y^{\prime}
$$

for definable $Y \subseteq \mathbb{R}^{m}, Y^{\prime} \subseteq \mathbb{R}^{n}$.

Proof. Let $\pi$ be the coordinate projection $\mathbb{R}^{m} \times \mathbb{R}^{n} \rightarrow \mathbb{R}^{m}$. We first show that

$$
\operatorname{dim} X \geq \operatorname{dim}\left\{a \in \mathbb{R}^{m}: \operatorname{dim} X_{a}=d\right\}+d \text { for all } 0 \leq d \leq n .
$$

Fix $0 \leq d \leq n$. After replacing $X$ by

$$
\left[\left\{a \in \mathbb{R}^{m}: \operatorname{dim} X_{a}=d\right\} \times \mathbb{R}^{n}\right] \cap X
$$

we suppose $\operatorname{dim} X_{a}=d$ for all $a \in \pi(X)$. So we show $\operatorname{dim} X$ is at least $\operatorname{dim} \pi(X)+d$. We show that $\operatorname{dim} \operatorname{Cl}(X)$ satisfies the same inequality and apply Proposition 10.8 
Note that $\operatorname{dim} \operatorname{Cl}(X)_{a} \geq d$ for all $a \in \pi(X)$. For each $d \leq k \leq n$ let $Z[k]$ be the set of $a \in \pi(X)$ such that $\operatorname{dim} \operatorname{Cl}(X)_{a}=k$. Applying Corollary 10.12 we obtain $d \leq k \leq n$ such that $\operatorname{dim} Z[k]=\operatorname{dim} \pi(X)$. Applying Proposition 10.13 we obtain a definable open subset $U$ of $\mathbb{R}^{m}$ such that $U \cap Z[k]$ is constructible and has dimension $\operatorname{dim} \pi(X)$. In particular $U \cap Z[k]$ is $D_{\Sigma}$. It follows that $Y:=\left[(U \cap Z[k]) \times \mathbb{R}^{n}\right] \cap \mathrm{Cl}(X)$ is also $D_{\Sigma}$. Applying the second claim of Fact 10.15 we obtain

$$
\operatorname{dim} Y=\operatorname{dim}(U \cap Z[k])+k=\operatorname{dim} \pi(X)+k \geq \operatorname{dim} \pi(X)+d .
$$

As $Y$ is a subset of $\operatorname{Cl}(X)$ we have $\operatorname{dim} \operatorname{Cl}(X) \geq \operatorname{dim} \pi(X)+d$.

We now show that there is $0 \leq d \leq n$ such that

$$
\operatorname{dim} X \leq \operatorname{dim}\left\{a \in \mathbb{R}^{m}: \operatorname{dim} X_{a}=d\right\}+d .
$$

Applying Proposition 10.13 we obtain a definable open subset $U$ of $\mathbb{R}^{m} \times \mathbb{R}^{n}$ such that $Q:=U \cap X$ is constructible and has the same dimension as $X$. So $Q$ is $D_{\Sigma}$. Applying Fact 10.15 we obtain $0 \leq e \leq n$ such that

$$
\operatorname{dim} Q \leq \operatorname{dim}\left\{a \in \mathbb{R}^{m}: \operatorname{dim} Q_{a} \geq e\right\}+e \leq \operatorname{dim}\left\{a \in \mathbb{R}^{m}: \operatorname{dim} X_{a} \geq e\right\}+e .
$$

By Corollary 10.12 there is $e \leq d \leq n$ such that

$$
\operatorname{dim}\left\{a \in \mathbb{R}^{n}: \operatorname{dim} X_{a}=d\right\}=\operatorname{dim}\left\{a \in \mathbb{R}^{n}: \operatorname{dim} X_{a} \geq e\right\} .
$$

So

$$
\operatorname{dim} X \leq \operatorname{dim}\left\{a \in \mathbb{R}^{n}: \operatorname{dim} X_{a}=d\right\}+d .
$$

We now prove Theorem 10.17

Theorem 10.17. Let $X$ be a definable subset of $\mathbb{R}^{n}$ and $f$ be a definable map $X \rightarrow \mathbb{R}^{m}$. Then

$$
\operatorname{dim} X=\max _{0 \leq d \leq n} \operatorname{dim}\left\{a \in \mathbb{R}^{m}: \operatorname{dim} f^{-1}(\{a\})=d\right\}+d .
$$

Proof. Let $G$ be the set of $(a, b) \in \mathbb{R}^{m} \times \mathbb{R}^{n}$ such that $f(b)=a$. Then $G_{a}=f^{-1}(\{a\})$ for all $a \in \mathbb{R}^{m}$. Theorem 10.16 shows that

$$
\operatorname{dim} G=\max _{0 \leq d \leq n} \operatorname{dim}\left\{a \in \mathbb{R}^{m}: \operatorname{dim} f^{-1}(\{a\})=d\right\}+d .
$$

The coordinate projection $\mathbb{R}^{m} \times \mathbb{R}^{n} \rightarrow \mathbb{R}^{n}$ gives a definable bijection $G \rightarrow X$ so $\operatorname{dim} G=\operatorname{dim} X$ by Theorem 10.14 .

As an application of the dimension theory we prove Lemma 10.18, which is used in the proof of Theorem 2.19.

Lemma 10.18. Let $X, Y$ be nonempty definable subsets of $\mathbb{R}^{m} \times \mathbb{R}^{n}$ and let $\pi$ : $\mathbb{R}^{m} \times \mathbb{R}^{n} \rightarrow \mathbb{R}^{m}$ be the coordinate projection. Suppose there is a nonempty open subset $U$ of $\pi(X)$ such that $Y_{a}$ has interior in $X_{a}$ for all $a \in U$. Then $Y$ has interior in $X$.

Proof. We first reduce to the case when $U$ is an open subset of $\mathbb{R}^{m}$. After applying Fact 2.18 and Theorem 2.17 and shrinking $U$ if necessary we obtain $0 \leq d \leq m$, a nonempty definable open $V \subseteq \mathbb{R}^{d}$, and a coordinate projection $\rho: \mathbb{R}^{m} \rightarrow \mathbb{R}^{d}$ such that $\rho$ restricts to a homeomorphism $U \rightarrow V$. Let $g$ be the definable homeomorphism $U \times \mathbb{R}^{n} \rightarrow V \times \mathbb{R}^{n}$ given by $g(a, b)=(\rho(a), b)$. It suffices to show that $g(Y)$ 
has interior in $g(X)$. So we suppose that $U$ is an open subset of $\mathbb{R}^{m}$.

For all $a \in U$ let $W_{a}$ be the union of all open boxes $B$ in $\mathbb{R}^{n}$ such that $B \cap X_{a} \subseteq Y_{a}$ and $B \cap X_{a} \neq \emptyset$. So $W_{a} \cap X_{a}$ is the interior of $Y_{a}$ in $X_{a}$ for all $a \in U$. Let $W$ be the set of $(a, b) \in \mathbb{R}^{m} \times \mathbb{R}^{n}$ such that $b \in W_{a}$. Note that $W$ is definable and each $W_{a}$ is nonempty and open. So $\operatorname{dim} W_{a}=n$ for all $a \in U$. As $\operatorname{dim} U=m$ Theorem 10.16 shows that $\operatorname{dim} W=m+n$. Fact 10.5 shows that $W$ has interior in $\mathbb{R}^{m} \times \mathbb{R}^{n}$. As $W \cap X \subseteq Y$ we see that $Y$ has interior in $X$.

We briefly describe some results on metric dimensions. It follows from [40, Corollary 1.5] that if $\mathcal{R}$ expands $(\mathbb{R},<,+, \times)$ and $X \subseteq \mathbb{R}^{n}$ is definable then the Assouad dimension of $X$ agrees with $\operatorname{dim} X$. We prove a similar result for expansions of $\mathbb{R}_{\text {vec. Fact } 10.19}$ is the main theorem of [26].

Fact 10.19. Suppose $\mathcal{S}$ is a type $A$ expansion of $\mathbb{R}_{\mathrm{vec}}$ and let $X \subseteq \mathbb{R}^{n}$ be $D_{\Sigma}$. Then the Hausdorff dimension of $X$ agrees with $\operatorname{dim} X$.

We let $\operatorname{dim}_{\mathrm{H}} X$ be the Hausdorff dimension of a subset $X$ of $\mathbb{R}^{n}$.

Proposition 10.20. Suppose $\mathcal{R}$ expands $\mathbb{R}_{\mathrm{vec}}$ and let $X \subseteq \mathbb{R}^{n}$ be definable. Then the Hausdorff dimension of $X$ agrees with $\operatorname{dim} X$.

Proof. Hausdorff dimension is monotone and bounded below by topological dimension, so $\operatorname{dim} X \leq \operatorname{dim}_{\mathrm{H}} X \leq \operatorname{dim}_{\mathrm{H}} \mathrm{Cl}(X)$. The proposition follows by combining Fact 10.19 and Proposition 10.8

It is an open question whether Proposition 10.20 holds for NIP expansions of $(\mathbb{R},<$ $,+)$ by closed sets. As as countable set has Hausdorff dimension zero this is closely related to Question 2.3 .

10.1. Theorem E. We continue to suppose that $\mathcal{R}$ is a generically locally ominimal expansion of $(\mathbb{R},<,+)$. We apply the tools developed above to develop a "Zil'ber dichotomy" between generically locally o-minimal expansions that define a field structure on an interval and those in which definable sets and functions are generically affine. The o-minimal case follows from the Peterzil-Starchenko

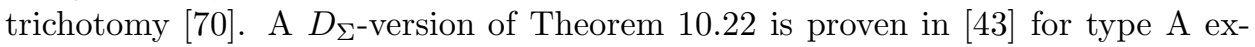
pansions. Our argument follows that of [43] but uses different tools.

We say that $\mathcal{R}$ is field-type if there is an open interval $I$ and definable $\oplus, \otimes: I^{2} \rightarrow I$ such that $(I,<, \oplus, \otimes)$ is an ordered field isomorphic to $(\mathbb{R},<,+, \times)$. This definition implies $\oplus, \otimes$ are continuous. It is easy to see that if $\mathcal{R}$ is field-type then there is a bounded open interval $I$ and $\oplus, \otimes: I^{2} \rightarrow I$ such that $(I,<, \oplus, \otimes)$ is isomorphic to $(\mathbb{R},<,+, \times)$. So we will always suppose that $I$ is bounded. We recall a general theorem from 43. (The case when $m=1$ is essentially due to Marker, Peterzil, and Pillay [59.)

Fact 10.21. Suppose $U$ is a nonempty connected open subset of $\mathbb{R}^{m}$ and $f: U \rightarrow \mathbb{R}^{n}$ is $C^{2}$ and not affine. Then $(\mathbb{R},<,+, f)$ is field-type.

A family $\mathcal{X}$ of one-dimensional subsets of $\mathbb{R}^{n}$ is normal if $\operatorname{dim} X \cap X^{\prime}=0$ for all distinct $X, X^{\prime} \in \mathcal{X}$. If $\left(X_{a}\right)_{a \in B}$ is a normal definable family of one-dimensional subsets of $\mathbb{R}^{n}$ then the dimension of $\left(X_{a}\right)_{a \in B}$ is defined to be the dimension of $B$. Given a subset $X$ of $\mathbb{R}^{n}$ we say that $p \in X$ is an affine point of $X$ if there is an 
open neighbourhood $U$ of $p$ such that $U \cap X=U \cap H$ for some affine subspace $H$ of $\mathbb{R}^{n}$. It is shown in [45] that the set of affine points of $X$ is definable in $(\mathbb{R},<,+, X)$.

Theorem 10.22. The following are equivalent

(1) $\mathcal{R}$ is field-type,

(2) there is a definable field $(Z, \oplus, \otimes)$ such that $\operatorname{dim} Z>0$,

(3) there is a definable normal family of one-dimensional subsets of $\mathbb{R}^{n}$ of dimension $\geq 2$,

(4) there is a definable $f: U \rightarrow \mathbb{R}^{m}$ where $U$ is an open subset of $\mathbb{R}^{n}$ and $f$ is nowhere locally affine,

(5) there is a definable subset $X$ of $\mathbb{R}^{n}$ such that the affine points of $X$ are not dense in $X$.

An o-minimal expansion of $(\mathbb{R},<,+)$ is field-type if and only if it is not a reduct of $\mathbb{R}_{\text {vec }}$ by the Peterzil-Starchenko trichotomy. This dichotomy fails for NIP expansions of $(\mathbb{R},<,+)$ by closed subsets of Euclidean space. Let $g: 2^{\mathbb{Z}} \times \mathbb{R} \rightarrow \mathbb{R}$ be given by $f\left(t, t^{\prime}\right)=t t^{\prime}$. Delon [15] studied $(\mathbb{R},<,+, g)$. It may be deduced from [15, Theorem 2] that $(\mathbb{R},<,+, g)$ is not field-type. However $(\mathbb{R},<,+, g)$ is NIP as it is a reduct of $\left(\mathbb{R},<,+, \times, 2^{\mathbb{Z}}\right)$.

Proof. A nonempty open interval is one-dimensonal so (1) implies (2).

$(2) \Rightarrow(3)$ : Let $(Z, \oplus, \otimes)$ be a definable field with $\operatorname{dim} Z \geq 1$. Suppose $Z$ is a subset of $\mathbb{R}^{n}$. Applying Corollary 10.9 we obtain a nonempty open interval $I$ and a definable injection $g: I \rightarrow Z$. Given $\left(a, a^{\prime}\right) \in I^{2}$ we let

$$
X_{\left(a, a^{\prime}\right)}:=\left\{\left(b, b^{\prime}\right) \in I \times Z: b^{\prime}=[g(a) \otimes g(b)] \oplus g\left(a^{\prime}\right)\right\} .
$$

Note that $X_{\left(a, a^{\prime}\right)}$ is the graph of the function $I \rightarrow Z$ given by

$$
x \mapsto[g(a) \otimes g(x)] \oplus g\left(a^{\prime}\right) .
$$

This function is injective so Theorem 10.14 shows that $\operatorname{dim} X_{\left(a, a^{\prime}\right)}=1$ for all $\left(a, a^{\prime}\right) \in I^{2}$. We show that $\left(X_{\left(a, a^{\prime}\right)}\right)_{\left(a, a^{\prime}\right) \in I^{2}}$ is normal. Let $\left(a, a^{\prime}\right)$ and $\left(b, b^{\prime}\right)$ be distinct elements of $I^{2}$. Then $(g(a), g(a))$ and $\left(g(b), g\left(b^{\prime}\right)\right)$ are distinct as $g$ is injective. Let $L_{\left(a, a^{\prime}\right)}$ be the graph of the function $Z \rightarrow Z$ given by $x \mapsto[g(a) \otimes x] \oplus g\left(a^{\prime}\right)$, likewise define $L_{\left(b, b^{\prime}\right)}$. Then $X_{\left(a, a^{\prime}\right)}$ and $X_{\left(b, b^{\prime}\right)}$ are contained in $L_{\left(a, a^{\prime}\right)}$ and $L_{\left(b, b^{\prime}\right)}$, respectively. As $(Z, \oplus, \otimes)$ is a field the intersection of $L_{\left(a, a^{\prime}\right)}$ and $L_{\left(b, b^{\prime}\right)}$ contains at most one element. So $X_{\left(a, a^{\prime}\right)} \cap X_{\left(b, b^{\prime}\right)}$ contains at most one element.

$(3) \Rightarrow(4)$ : Suppose $\left(X_{a}\right)_{a \in B}$ is a definable normal family of one-dimensional subsets of $\mathbb{R}^{n}$ where $B$ is a definable subset of $\mathbb{R}^{m}$ of dimension $\geq 2$. Applying Corollary 10.9 we obtain a nonempty definable open $U \subseteq \mathbb{R}^{2}$ and a definable injection $f: U \rightarrow B$. After replacing $\left(X_{a}\right)_{a \in B}$ with $\left(X_{f(a)}\right)_{a \in U}$ if necessary we suppose $B$ is an open subset of $\mathbb{R}^{2}$.

Let $\pi_{k}: \mathbb{R}^{n} \rightarrow \mathbb{R}$ be the projection onto the $k$ th coordinate for $1 \leq k \leq n$. As $\operatorname{dim} X_{a}=1$ for all $a \in B$ an application of Theorem 10.10 shows that for any $a \in B$ there is $1 \leq k \leq n$ such that $\pi_{k}\left(X_{a}\right)$ has interior. Given $1 \leq k \leq n$ let $B_{k}$ be the set of $a \in B$ such that $\pi_{k}\left(X_{a}\right)$ has interior. Then there is $1 \leq k \leq n$ such that $B_{k}$ is somewhere dense in $B$. We assume that $B_{1}$ is somewhere dense in $B$, the case when $B_{k}$ is somewhere dense for $2 \leq k \leq n$ follows in the same way. So $B_{1}$ has interior in 
$B$. After replacing $B$ with a smaller nonempty definable open subset of $\mathbb{R}^{2}$ if necessary we suppose $\pi_{1}\left(X_{a}\right)$ has interior for all $a \in B$. Let $\rho: B \times \mathbb{R}^{n} \rightarrow B \times \mathbb{R}$ be the projection onto the first three coordinates. Then $\rho(X)_{a}=\pi_{1}\left(X_{a}\right)$ for all $a \in B$. As $\rho(X)_{a}$ has interior for all $a \in B$ an application of Lemma 10.18 shows that $\rho(X)$ has interior. Let $V$ be a definable open subset of $\mathbb{R}^{3}$ contained in $\rho(X)$. Applying definable selection we obtain a definable $g: V \rightarrow \mathbb{R}^{n-1}$ such that $(c, g(a, b, c)) \in X_{(a, b)}$ for all $(a, b, c) \in V$.

We show that $g$ is nowhere locally affine. Suppose otherwise. After shrinking $V$ if necessary we suppose $g$ is affine and $V$ is of the form $I_{1} \times I_{2} \times I_{3}$ for nonempty open intervals $I_{1}, I_{2}, I_{3}$. Fix linear $h_{1}, h_{2}, h_{3}: \mathbb{R} \rightarrow \mathbb{R}^{n-1}$ and $\beta \in \mathbb{R}^{n-1}$ such that

$$
g\left(a_{1}, a_{2}, a_{3}\right)=h_{1}\left(a_{1}\right)+h_{2}\left(a_{2}\right)+h_{3}\left(a_{3}\right)+\beta \text { for all }\left(a_{1}, a_{2}, a_{3}\right) \in I_{1} \times I_{2} \times I_{3} .
$$

Fix $(u, v) \in I_{1} \times I_{2}$ and $u^{\prime} \in I_{1}$ such that $u^{\prime} \neq u$. Let $v^{\prime} \in I_{2}$ satisfy

$$
h_{1}\left(u^{\prime}\right)+h_{2}\left(v^{\prime}\right)=h_{1}(v)+h_{2}(v) .
$$

Then $f(u, v, t)=f\left(u^{\prime}, v^{\prime}, t\right)$ for all $t \in I_{3}$. So $\left\{(t, f(u, v, t)): t \in I_{3}\right\}$ is a subset of both $X_{(u, v)}$ and $X_{\left(u^{\prime}, v^{\prime}\right)}$. As $\left\{(t, f(u, v, t)): t \in I_{3}\right\}$ is one-dimensional we obtain a contradiction with our assumption that $\left(X_{a}\right)_{a \in B}$ is normal.

$(4) \Rightarrow(5)$ : First observe that if $V$ is an open subset of $\mathbb{R}^{n}$ and $f: V \rightarrow \mathbb{R}^{m}$ is continuous then $f$ is locally affine at $p \in V$ if and only if $(p, f(p))$ is an affine point of $\operatorname{gr}(f)$. Now suppose $U$ is a definable open subset of $\mathbb{R}^{n}$ and $f: U \rightarrow \mathbb{R}^{m}$ is definable such that $f$ is nowhere locally affine. After applying Theorem 10.3 and shrinking $U$ if necessary we suppose $f$ is continuous. Then $\operatorname{gr}(f)$ does not have any affine points.

$(5) \Rightarrow(1)$ : Suppose $X \subseteq \mathbb{R}^{n}$ is definable and suppose that the affine points of $X$ are not dense in $X$. Applying Theorem 10.4 we obtain a definable open subset $U$ of $\mathbb{R}^{n}$ and $0 \leq d \leq n$ such that $U \cap X$ is a $d$-dimensional $C^{2}$-submanifold of $\mathbb{R}^{n}$. After permuting coordinates and shrinking $U$ if necessary we obtain a nonempty open $V \subseteq \mathbb{R}^{d}$ and a $C^{2}$ definable $f: V \rightarrow \mathbb{R}^{n-d}$ such that $U \cap X=\operatorname{gr}(f)$. We also may suppose that $U$ and $V$ are both connected. Our assumption on $X$ implies that $f$ is not affine. Fact 10.21 shows that $\mathcal{R}$ is field-type.

In the d-minimal case we get a sharper version of Theorem 10.22

Proposition 10.23. Suppose $\mathcal{R}$ is d-minimal. Then the following are equivalent.

(1) $\mathcal{R}$ does not interpret $(\mathbb{R},<,+, \times)$,

(2) $\mathcal{R}$ is not field type,

(3) if $X$ is a definable subset of $\mathbb{R}^{n}$ then there are definable locally affine subsets $Y_{0}, \ldots, Y_{k}$ of $\mathbb{R}^{n}$ such that $X=Y_{0} \cup \ldots \cup Y_{k}, Y_{0}$ is open and dense in $X$, $Y_{i}$ is open and dense in $X \backslash\left(Y_{0} \cup \ldots \cup Y_{i-1}\right)$ for all $1 \leq i \leq k$.

(4) every definable subset of every $\mathbb{R}^{n}$ is a finite union of locally affine sets.

Note that Delon's structure $(\mathbb{R},<,+, g)$ discussed after the statement of Theorem 10.22 is d-minimal as it is a reduct of $\left(\mathbb{R},<,+, \times, 2^{\mathbb{Z}}\right)$.

Proof. It is clear that (1) implies (2).

$(2) \Rightarrow(3)$ : Suppose $X$ is a definable subset of $\mathbb{R}^{n}$. We apply induction on the Pillay rank of $\mathrm{Cl}(X)$. If $\operatorname{Pr}(\mathrm{Cl}(X))=-1$ then $X$ is empty and hence locally affine. 
Suppose $\operatorname{Pr}(\operatorname{Cl}(X)) \geq 1$. Let $Y_{0}$ be the set of affine points of $X$. Then $Y_{0}$ is locally affine, definable, and open in $X$. Theorem 10.22 shows that $Y_{0}$ is dense in $X$ so $X \backslash Y_{0}$ is nowhere dense in $X$. It follows that $\mathrm{Cl}\left(X \backslash Y_{0}\right)$ is nowhere dense in $\mathrm{Cl}(X)$ so $\operatorname{Pr}\left(\mathrm{Cl}\left(X \backslash Y_{0}\right)\right)<\operatorname{Pr}(\mathrm{Cl}(X))$. Applying induction we obtain definable locally affine sets $Y_{1}, \ldots, Y_{k}$ such that $X \backslash Y_{0}=\bigcup_{i=1}^{k} Y_{i}$ and $Y_{i}$ is open and dense in $X \backslash\left(Y_{0} \cup \ldots \cup Y_{i-1}\right)$ for all $1 \leq i \leq k$.

It is clear that (3) implies (4). We show that (4) implies (2). Suppose $\mathcal{R}$ is field type. Applying Theorem 10.22 we obtain a nonempty definable open subset $U$ of $\mathbb{R}^{m}$ and a definable $f: U \rightarrow \mathbb{R}^{n}$ which is nowhere locally affine. After applying Theorem 10.3 we suppose $f$ is continuous so $\operatorname{gr}(f)$ is homeomorphic to $U$. We show that $\operatorname{gr}(f)$ is not a finite union of locally affine sets. A locally affine subset of $\mathbb{R}^{m+n}$ is contained in a countable union of affine subspaces of $\mathbb{R}^{m+n}$, so a finite union of locally affine subsets is contained in a countable union of affine subspaces. So it suffices to suppose $\left\{H_{i}\right\}_{i \in \mathbb{N}}$ is a sequence of affine subspaces of $\mathbb{R}^{m+n}$ and show that $\bigcup_{i \in \mathbb{N}} H_{i}$ does not contain $\operatorname{gr}(f)$. If $(p, f(p)) \in \operatorname{gr}(f)$ lies in the interior of $H_{i}$ in $\operatorname{gr}(f)$ then $f$ is locally affine at $p$. So each $H_{i}$ has empty interior in $\operatorname{gr}(f)$. As $H_{i}$ is closed it follows that each $H_{i}$ is nowhere dense in $\operatorname{gr}(f)$. As $\operatorname{gr}(f)$ is homeomorphic to $U$ an application of the Baire category theorem shows that $\bigcup_{i \in \mathbb{N}} H_{i}$ has empty interior in $\operatorname{gr}(f)$.

$(2) \Rightarrow(1)$ : Suppose $\mathcal{R}$ is not field-type. By Fact 10.1 it suffices to show that $\mathcal{R}$ does not define an isomorphic copy of $(\mathbb{R},<,+, \times)$. Suppose $(Z, \oplus, \otimes)$ is a definable field. Theorem 10.22 shows that $\operatorname{dim} Z=0$. Applying Fact 2.22 we see that $Z$ is countable so $(Z, \oplus, \otimes)$ is not isomorphic to $(\mathbb{R},+, \times)$.

If $\mathcal{R}$ is o-minimal then a definable set is zero-dimensional if and only if it is finite. In this case condition (2) of Theorem 10.22 is equivalent to the assumption that $\mathcal{R}$ does not define an infinite field. The expansion of $(\mathbb{R},<,+)$ by all subsets of all $\mathbb{Z}^{n}$ is locally o-minimal and not field-type and obviously defines an isomorphic copy of any countable field, see Fact 2.1.

Conjecture 4. Suppose $\mathcal{R}$ is an NIP expansion of $(\mathbb{R},<,+)$ by closed subsets of Euclidean space. If $\mathcal{R}$ interprets an infinite field then $\mathcal{R}$ is field-type.

By Fact 10.1 we may replace "interprets" with "defines" in this conjecture. Conjecture 4 fails for noisey NIP expansions. Let $\mathcal{F}$ be an NIP (strongly dependent) field of cardinality $\leq 2^{\aleph_{0}}$. Then there is a noisey NIP (strongly dependent) expansion $\mathcal{R}$ of $(\mathbb{R},<,+)$ such that $\mathcal{R}$ interprets $\mathcal{F}$ and $\mathcal{R}^{\circ}$ is interdefinable with $(\mathbb{R},<,+)$ [42. (Note that an expansion $\mathcal{R}$ of $(\mathbb{R},<,+)$ is field-type if and only if $\mathcal{R}^{\circ}$ is field type.)

Proposition 10.24 handles the positive characteristic case of Conjecture 5. This proposition was observed for noiseless NIP expansions in [42, we include a proof for the sake of completeness.

Proposition 10.24. Suppose $\mathcal{R}$ is an NIP expansion of $(\mathbb{R},<,+)$ by closed subsets of Euclidean space. Then $\mathcal{R}$ does not interpret an infinite vector space over a finite field. In particular $\mathcal{R}$ does not interpret an infinite field of positive characteristic.

Proof. Fix a finite field $\mathbb{F}$. Let $\mathcal{V}$ be an infinite $\mathbb{F}$-vector space. Suppose $\mathcal{V}$ is interpretable in $\mathcal{R}$. By Fact 10.1 we may suppose that $\mathcal{V}$ is definable in $\mathcal{R}$. Suppose 
the domain of $\mathcal{V}$ is a subset of $\mathbb{R}^{m}$. Let $\prec$ be the lexicographic order on $\mathbb{R}^{m}$. Shelah and Simon [73, Theorem 2.1] show that any expansion of an infinite $\mathbb{F}$-vector space by a linear order is IP. So $(\mathcal{V}, \prec)$ is IP, contradiction.

We prove several more special cases of Conjecture 4. In particular we prove the strongly dependent case in Proposition 11.12, We do this by establishing special cases of a stronger conjecture, Conjecture 5. Theorem 10.22 shows that Conjecture 4 follows from Conjecture 5

Conjecture 5. An NIP expansion of $(\mathbb{R},<,+)$ by closed subsets of Euclidean space cannot define a field $(Z, \oplus, \otimes)$ where $Z$ is infinite and zero-dimensional.

We will need Proposition 10.25

Proposition 10.25. No infinite field is interpretable in $(\mathbb{Z},<,+)$.

Proof. We are aware of two approaches to Proposition 10.25. As $(\mathbb{Z},<,+)$ eliminates imaginaries it suffices to show that $(\mathbb{Z},<,+)$ does not define an infinite field. Onshuus and Vicaria [69] show that any group definable in $(\mathbb{Z},<,+)$ has an abelian subgroup of finite index. It is basic fact from algebra that if $K$ is an infinite field then $\mathrm{Sl}_{2}(K)$ does not have a finite index abelian subgroup. Alternatively, as $(\mathbb{Z},<,+)$ is dp-minimal any structure definable in $(\mathbb{Z},<,+)$ has finite dp rank, Dolich and Goodrick [17, Corollary 2.2] show that an expansion of a field which does not eliminate $\exists^{\infty}$ has infinite dp rank, and it is easy to see that the structure induced on any infinite subset of $\mathbb{Z}^{n}$ by $(\mathbb{Z},<,+)$ does not eliminate $\exists^{\infty}$.

Proposition 10.26 and Theorem 10.22 yield Conjecture 4 for reducts of $\left(\mathcal{S}, \lambda^{\mathbb{Z}}\right)$. In particular the structure $(\mathbb{R},<,+, g)$ discussed after Theorem 10.22 does not interpret an infinite field.

Proposition 10.26. Suppose $\mathcal{S}$ is an o-minimal expansion of $(\mathbb{R},<,+, \times)$ with rational exponents and suppose $\lambda>1$. Then $\left(\mathcal{S}, \lambda^{\mathbb{Z}}\right)$ does not define an infinite zero-dimensional field.

Proof. Tychonievich [82, 4.1.10] shows that if $X \subseteq \mathbb{R}^{n}$ is zero-dimensional and definable in $\left(\mathcal{S}, \lambda^{\mathbb{Z}}\right)$ then there is an $\left(\mathcal{S}, \lambda^{\mathbb{Z}}\right)$-definable surjection $\left(\lambda^{\mathbb{Z}}\right)^{m} \rightarrow X$ for some $m$. Tychonievich [82, 4.1.2] also shows that the structure induced on $\lambda^{\mathbb{Z}}$ by $\left(\mathcal{S}, \lambda^{\mathbb{Z}}\right)$ is interdefinable with $\left(\lambda^{\mathbb{Z}},<, \times\right)$. As $\left(\lambda^{\mathbb{Z}},<, \times\right)$ is isomorphic to $(\mathbb{Z},<,+)$ the proposition follows from Proposition 10.25 .

Similar approaches should yield Conjecture 4 for other expansions, we briefly describe one example. Fix $\lambda>1$, let $D$ be $\left\{\lambda, \lambda^{\lambda}, \lambda^{\lambda^{\lambda}}, \ldots\right\}$, and let $\mathcal{S}$ be any o-minimal expansion of $(\mathbb{R},<,+, \times)$ such that every $\mathcal{S}$-definable function $\mathbb{R} \rightarrow \mathbb{R}$ is eventually bounded above by some compositional iterate of the exponential (every known ominimal expansion satisfies this condition). Miller and Tyne 66] show that $(\mathcal{S}, D)$ admits quantifier elimination in a natural language and is d-minimal. It should follow that $(\mathcal{S}, D)$ is NIP, every zero-dimensional $(\mathcal{S}, D)$-definable set is a definable image of some $D^{n}$, and the induced structure on $D$ is interdefinable with $(D,<)$. The proof of Proposition 10.26 would then show that $(\mathcal{S}, D)$ does not define an infinite zero-dimensional field.

We describe two closely related conjectures which should be more approachable. Conjecture 6 is a special case of Conjecture 7 
ERIK WALSBERG

Conjecture 6. Suppose $(\mathbb{N}, \oplus, \otimes)$ is a field. Then $(\mathbb{N},<, \oplus, \otimes)$ is IP.

It is likely that if Conjecture 6 fails then Conjecture 4 also fails. Consider $(\mathbb{R},<$ ,,$\left.+ 2^{\mathbb{N}}\right)$. We expect that the structure induced on $2^{\mathbb{N}}$ by $\left(\mathbb{R},<,+, 2^{\mathbb{N}}\right)$ is interdefinable with $\left(2^{\mathbb{N}},<\right)$. If $\left(2^{\mathbb{N}}, \oplus, \otimes\right)$ is a field then $\left(\mathbb{R},<,+, 2^{\mathbb{N}}, \oplus, \otimes\right)$ is d-minimal by 30 . If $\left(2^{\mathbb{N}}, \oplus, \otimes\right)$ is in addition NIP then we expect $\left(\mathbb{R},<,+, 2^{\mathbb{N}}, \oplus, \otimes\right)$ to be NIP as well.

A natural weakening of Conjecture $[$ is that $(\mathbb{N},<,+, \oplus, \otimes)$ is IP when $(\mathbb{N}, \oplus, \otimes)$ is a field. If this weaker conjecture fails then Fact 2.1 may be applied to construct a locally o-minimal expansion which is not field-type and defines an infinite field. The theorem of Dolich and Goodrick described in the proof of Proposition 10.25 shows that $(\mathbb{N},<, \oplus, \otimes)$ has infinite dp-rank if $(\mathbb{N}, \oplus, \otimes)$ is a field. However there are NIP expansions of fields which do not eliminate $\exists^{\infty}$ such as $\left(\mathbb{R},<,+, \times, \lambda^{\mathbb{Z}}\right)$.

Conjecture 7. If $\mathcal{F}$ is a expansion of an infinite field which admits a definable directed family $\mathcal{X}$ of finite subsets of $F$ such that $\bigcup \mathcal{X}=F$ then $\mathcal{F}$ is IP.

Conjecture 7 implies the d-minimal case of Conjecture 5 as it is not difficult to show that if $\mathcal{R}$ is $\mathrm{d}$-minimal and $X$ is a zero-dimensional definable subset of $\mathbb{R}^{n}$ then there is a definable directed family $\mathcal{X}$ of finite subsets of $\mathbb{R}^{n}$ such that $\bigcup \mathcal{X}=X$. It may also be worth observing that Conjecture 7 implies that an NIP expansion of $(\mathbb{N},<)$ cannot interpret an infinite field.

The proof of Proposition 10.24 is easily adapted to show that $(\mathbb{N},<, \oplus, \otimes)$ is IP when $(\mathbb{N}, \oplus, \otimes)$ is a field of positive characteristic. Proposition 10.27 follows from the proof of Proposition 5.6 .

Proposition 10.27. Suppose $\mathcal{F}$ is a expansion of an infinite field which admits both a definable non-discrete field topology and a definable directed family $\mathcal{X}$ of finite subsets of $F$ such that $\bigcup \mathcal{X}=F$. Then $\mathcal{F}$ is $\mathrm{TP}_{2}$.

Every known unstable NIP expansion of a field admits a definable non-discrete field topology. Johnson [49 shows that an unstable dp-minimal expansion of a field admits a definable non-discrete field topology. Conjecture 7 would follow from Proposition 10.27 and a generalization of Johnson's theorem. This is probably not the right way to go about establishing Conjecture 7

\section{Strongly Dependent Expansions of $(\mathbb{R},<,+)$}

We continue to suppose that $\mathcal{R}$ is an expansion of $(\mathbb{R},<,+)$. In this section we analyse strongly dependent expansions of $(\mathbb{R},<,+)$ by closed sets and in particular prove Theorem $\mathrm{D}$. We first discuss $(\mathbb{R},<,+, \alpha \mathbb{Z})$-minimality. We then show that a strongly dependent expansion of $(\mathbb{R},<,+)$ by closed sets is either o-minimal or $(\mathbb{R},<,+, \alpha \mathbb{Z}$ )-minimal for some $\alpha>0$. (This $\alpha$ is unique up to rational multiples.) As an application we prove the strongly dependent case of Conjecture [5]

We will make crucial use of the work of Dolich and Goodrick on strongly dependent expansions of ordered abelian groups [17. Dolich and Goodrick work in the more general setting of strong theories. So the reader may wonder if our results on strongly dependent expansions of $(\mathbb{R},<,+)$ generalize to strong expansions. This is true and will appear in forthcoming joint work with Hieronymi. We do not know if an $\mathrm{NTP}_{2}$ expansion of $(\mathbb{R},<,+)$ by closed sets is generically locally o-minimal. 
11.1. $(\mathbb{R},<,+, \mathbb{Z})$-minimality. Recall that $\mathcal{R}$ is $(\mathbb{R},<,+, \mathbb{Z})$-minimal if it expands $(\mathbb{R},<,+, \mathbb{Z})$ and every $\mathcal{R}$-definable subset of $\mathbb{R}$ is $(\mathbb{R},<,+, \mathbb{Z})$-definable. Equivalently: $\mathcal{R}$ is $(\mathbb{R},<,+, \mathbb{Z})$-minimal if the $\mathcal{R}$-definable subsets of $\mathbb{R}$ are all finite unions of sets of the form $A+I$ where $A=s+t \mathbb{N}$ for some $s \in \mathbb{R}, t \in \mathbb{Q}$ and interval $I$. Note that $(\mathbb{R},<,+, \mathbb{Z})$-minimality implies local o-minimality. So we may apply the following result of Kawakami, Takeuchi, Tanaka, and Tsuboi [51] on locally o-minimal expansions of $(\mathbb{R},<,+)$ which define $\mathbb{Z}$.

For each $n$ let $\iota_{n}$ be the bijection $[0,1)^{n} \times \mathbb{Z}^{n} \rightarrow \mathbb{R}^{n}$ given by $\iota_{n}(a, d)=a+d$ and let $+_{1}$ be the function $[0,1)^{2} \rightarrow[0,1)$ given by declaring $t+{ }_{1} t^{\prime}=t+t^{\prime}$ when $t+t^{\prime}<1$ and $t+{ }_{1} t^{\prime}=t+t^{\prime}-1$ otherwise. So for any $A \subseteq[0,1)^{n}$ and $B \subseteq \mathbb{Z}^{n}$ we have

$$
\iota_{n}(A \times B)=\bigcup_{b \in B} A+b .
$$

Fact 11.1 is [51, Lemma 23, Theorem 24]. Fact 11.1 shows that any locally ominimal expansion of $(\mathbb{R},<,+)$ which defines $\mathbb{Z}$ is bi-interpretable with the disjoint union of an o-minimal expansion of $\left([0,1),<,+_{1}\right)$ and an expansion of $(\mathbb{Z},<,+)$. Fact 11.1 is a converse to Fact 2.1. Informally Fact 11.1 shows that a locally ominimal expansion which defines $\mathbb{Z}$ may be decomposed into a "fractional part" and an "integer part". This is a higher order analogue of the decomposition of a real number into a fractional part and an integer part.

Fact 11.1. Suppose $\mathcal{R}$ is locally o-minimal and defines $\mathbb{Z}$. Every definable subset $[0,1)^{n} \times \mathbb{Z}^{n}$ is a finite union of sets of the form $A \times B$ for definable $A \subseteq[0,1)^{n}$ and $B \subseteq \mathbb{Z}^{n}$, hence every definable subset of $\mathbb{R}^{n}$ is a finite union of sets of the form

$$
\iota_{n}(A \times B)=\bigcup_{b \in B} A+b
$$

for definable $A \subseteq[0,1)^{n}$ and $B \subseteq \mathbb{Z}^{n}$. Therefore $\mathcal{R}$ is bi-interpretable with the disjoint union of $\mathcal{J}$ and $\mathcal{D}$ where $\mathcal{J}$ is the structure induced on $[0,1)$ by $\mathcal{R}$ and $\mathcal{D}$ is the structure induced on $\mathbb{Z}$ by $\mathcal{R}$. Note that $\mathcal{J}$ is o-minimal as $\mathcal{R}$ is locally o-minimal.

We also apply Fact 11.2, a theorem of Michaux and Villemaire 61.

Fact 11.2. There are no proper $(\mathbb{Z},+,<)$-minimal expansions of $(\mathbb{Z},+,<)$.

We can now prove Proposition 11.3 ,

Proposition 11.3. Suppose $\mathcal{R}$ is $(\mathbb{R},<,+, \mathbb{Z})$-minimal. Then every $\mathcal{R}$-definable subset of $\mathbb{R}^{n}$ is a finite union of sets of the form $\bigcup_{b \in B} b+A$ where $A$ is an $\mathcal{R}$ definable subset of $[0,1)^{n}$ and $B$ is a $(\mathbb{Z},<,+)$-definable subset of $\mathbb{Z}^{n}$. In particular any subset of $\mathbb{Z}^{n}$ definable in $\mathcal{R}$ is definable in $(\mathbb{Z},<,+)$. It follows that $\mathcal{R}$ is biinterpretable with the disjoint union of the induced structure on $[0,1)$ and $(\mathbb{Z},<,+)$.

Strong dependence is preserved under disjoint unions and bi-interpretations, so Proposition 11.3 shows that $(\mathbb{R},<,+, \mathbb{Z})$-minimality implies strong dependence.

Proof. By Fact 11.1 it suffices to show that the structure induced on $\mathbb{Z}$ by $\mathcal{R}$ is interdefinable with $(\mathbb{Z},<,+)$. It follows from the description of $(\mathbb{R},<,+, \mathbb{Z})$-definable sets that every subset $\mathcal{R}$-definable of $\mathbb{Z}$ is $(\mathbb{Z},<,+)$-definable. Apply Fact 11.2 .

We now classify $(\mathbb{R},<,+, \alpha \mathbb{Z})$-minimal structures. Suppose $\mathcal{S}$ is an o-minimal expansion of $(\mathbb{R},<,+)$. A pole is an $\mathcal{S}$-definable surjection from a bounded interval to 
an unbounded interval. We say that $\mathcal{S}$ has rational scalars if the function $\mathbb{R} \rightarrow \mathbb{R}$ given by $t \mapsto \lambda t$ is only definable when $\lambda \in \mathbb{Q}$.

Theorem 11.4. Fix $\alpha>0$. The following are equivalent:

(1) $\mathcal{R}$ is $(\mathbb{R},<,+, \alpha \mathbb{Z})$-minimal,

(2) There is a collection $\mathcal{B}$ of bounded subsets of Euclidean space such that $(\mathbb{R},<,+, \mathcal{B})$ is o-minimal and $\mathcal{R}$ is interdefinable with $(\mathbb{R},<,+, \mathcal{B}, \alpha \mathbb{Z})$,

(3) There is an o-minimal expansion $\mathcal{S}$ of $(\mathbb{R},<,+)$ with has no poles and has rational scalars such that $\mathcal{R}$ is interdefinable with $(\mathcal{S}, \alpha \mathbb{Z})$.

(4) There is an o-minimal expansion $\mathcal{S}$ of $(\mathbb{R},<,+)$ such that $(\mathcal{S}, \alpha \mathbb{Z})$ is locally $o$-minimal and $\mathcal{R}$ is interdefinable with $(\mathcal{S}, \alpha \mathbb{Z})$.

As $\left(\mathbb{R},<,+,\left.\sin \right|_{[0, \pi]}\right)$ is o-minimal and $(\mathbb{R},<,+, \sin ),\left(\mathbb{R},<,+,\left.\sin \right|_{[0, \pi]}, \pi \mathbb{Z}\right)$ are interdefinable, we see that $(\mathbb{R},<,+, \sin )$ is $(\mathbb{R},<,+, \pi \mathbb{Z})$-minimal. The equivalence of (2) and (3) follows from Fact 11.5, a special case of a theorem of Edmundo [21.

Fact 11.5. The following are equivalent for an o-minimal expansion $\mathcal{S}$ of $(\mathbb{R},<,+)$,

- S has no poles and has rational scalars,

- $\mathcal{S}$ is interdefinable with $(\mathbb{R},<,+, \mathcal{B})$ for some collection $\mathcal{B}$ of bounded subsets of Euclidean space.

We now prove Theorem 11.4

Proof. Rescaling reduces to the case $\alpha=1$. We first show (1) and (2) are equivalent.

Suppose that $\mathcal{R}$ is $(\mathbb{R},<,+, \mathbb{Z})$-minimal. Let $\mathcal{B}$ be the collection of all $\mathcal{R}$-definable subsets of all $[0,1)^{n}$. As $\mathcal{R}$ is locally o-minimal Theorem 2.13 shows that $(\mathbb{R},<,+, \mathcal{B})$ is o-minimal. It is immediate that $(\mathbb{R},<,+, \mathcal{B}, \mathbb{Z})$ is a reduct of $\mathcal{R}$. Proposition 11.3 shows that $\mathcal{R}$ is a reduct of $(\mathbb{R},<,+, \mathcal{B}, \mathbb{Z})$.

Now suppose (2). Rescaling and translating reduces to the case when every element of $\mathcal{B}$ is a subset of some $[0,1)^{n}$. Let $\mathcal{J}$ be the structure induced on $[0,1)$ by $(\mathbb{R},<,+, \mathcal{B})$. Note that $\mathcal{J}$ is an o-minimal expansion of $\left([0,1),<,+_{1}\right)$. Let $\mathcal{S}$ be the expansion of $(\mathbb{R},<,+)$ constructed from $\mathcal{J}$ and $(\mathbb{Z},<,+)$ as in Fact 2.1. The description of $\mathcal{S}$-definable sets in Fact 2.1 shows that $\mathcal{S}$ is $(\mathbb{R},<,+, \mathbb{Z})$-minimal. It is immediate that $(\mathbb{R},<,+, \mathcal{B}, \mathbb{Z})$ is a reduct of $\mathcal{S}$ and the description of $\mathcal{S}$-definable sets in Fact 2.1 shows that $\mathcal{S}$ is a reduct of $(\mathbb{R},<,+, \mathcal{B}, \mathbb{Z})$. So $\mathcal{S}$ and $(\mathbb{R},<,+, \mathcal{B}, \mathbb{Z})$ are interdefinable. It follows that $(\mathbb{R},<,+, \mathcal{B}, \mathbb{Z})$ is $(\mathbb{R},<,+, \mathbb{Z})$-minimal.

Fact 11.5 shows that (2) and (3) are equivalent. So (1) and (3) are equivalent, it follows that (3) implies (4) as $(\mathbb{R},<,+, \alpha \mathbb{Z})$-minimality implies local o-minimality. We show that (4) implies (3) by showing that if $\mathcal{S}$ is an o-minimal expansion of $(\mathbb{R},<,+)$ and $(\mathcal{S}, \mathbb{Z})$ is locally o-minimal then $\mathcal{S}$ has no poles and has rational scalars.

Suppose $\tau: I \rightarrow J$ is a pole. Applying the monotonicity theorem for o-minimal structures, reflecting, and translating, we suppose that $J$ contains a final segment of $\mathbb{R}, \tau$ is strictly increasing, and $\tau$ is continuous. Then $\tau^{-1}(\mathbb{N})$ is an infinite discrete subset of a bounded interval so $(\mathcal{S}, \mathbb{Z})$ is not locally o-minimal. Suppose $\lambda \in \mathbb{R}$ is irrational and the map $\mathbb{R} \rightarrow \mathbb{R}, t \mapsto \lambda t$ is $\mathcal{S}$-definable. Then $\mathbb{Z}+\lambda \mathbb{Z}$ is dense and co-dense in $\mathbb{R}$ so $(\mathcal{S}, \mathbb{Z})$ is not locally o-minimal. 
11.2. Strong dependence. We now show that strongly dependent expansions of $(\mathbb{R},<,+)$ by closed sets are either o-minimal or $(\mathbb{R},<,+, \alpha \mathbb{Z})$-minimal for some $\alpha>0$ which is unique up to rational multiples. We need (special cases of) several results of Dolich and Goodrick. The first claim of Fact 11.6] is a special case of [17, Corollary 2.13]. The second claim is a essentially a case of [17, Theorem 2.18].

Fact 11.6. Suppose that $\mathcal{R}$ is strongly dependent and $E \subseteq \mathbb{R}$ is definable and discrete. Then $E$ has no accumulation points. Furthermore there are $s_{1}, \ldots, s_{n}, t_{1}, \ldots, t_{n}$ such that $E=\left(s_{1}+t_{1} \mathbb{N}\right) \cup \ldots \cup\left(s_{n}+t_{n} \mathbb{N}\right)$ and $t_{i} / t_{j} \in \mathbb{Q}$ for all $i, j$ such that $t_{j} \neq 0$.

We say that the second claim is "essentially" a special case of [17, Theorem 2.18] because that theorem is slightly incorrect as stated. We explain. Dolich and Goodrick assert, under the assumptions of Fact 11.6, that $E$ is a union of a finite set together with commensurable progressions $s_{1}+t_{1} \mathbb{N}, \ldots, s_{n}+t_{n} \mathbb{N}$. (Recall $s_{1}+t_{1} \mathbb{N}$ and $s_{2}+t_{2} \mathbb{N}$ are commensurable if they both lie in $s+t \mathbb{Z}$ for some $s, t$.) This need not be the case, for example $\mathbb{N}$ and $\alpha+\mathbb{N}$ are not commensurable for any $\alpha \in \mathbb{R} \backslash \mathbb{Q}$. The mistake lies in [17, Corollary 2.30]. In the proof of that corollary it is asserted that if $s_{1}+t_{1} \mathbb{N}, s_{2}+t_{2} \mathbb{N}$ are both infinite and contained in $\mathbb{R}_{>0}$ and

$$
\left\{t-\min \left\{r \in s_{1}+t_{1} \mathbb{N}, s_{2}+t_{2} \mathbb{N}: r>t\right\}: t \in s_{1}+t_{1} \mathbb{N}, s_{2}+t_{2} \mathbb{N}\right\}
$$

is disjoint from $[0, \varepsilon)$ for some $\varepsilon>0$ then $s_{1}+t_{1} \mathbb{N}$ and $s_{2}+t_{2} \mathbb{N}$ are commensurable. This is incorrect, but it does imply that $t_{1} / t_{2} \in \mathbb{Q}$. The rest of the proof is correct].

Fact 11.7 is a theorem of Bès and Choffrut $[6$. We only need the case $n=1$. The case $n=1$ was also proven independently by Miller and Speissegger 64.

Fact 11.7. Suppose $X$ is a subset of $\mathbb{R}^{n}$ which is definable in $(\mathbb{R},<,+, \mathbb{Z})$ and not definable in $(\mathbb{R},<,+)$. Then $(\mathbb{R},<,+, X)$ defines $\mathbb{Z}$.

We can now describe strongly dependent expansions of $(\mathbb{R},<,+)$ by closed sets.

Theorem 11.8. The following are equivalent:

(1) $\mathcal{R}$ is a strongly dependent expansion by closed sets.

(2) $\mathcal{R}$ is strongly dependent and noiseless.

(3) $\mathcal{R}$ is either o-minimal or $(\mathbb{R},<,+, \alpha \mathbb{Z})$-minimal for some $\alpha>0$.

(4) $\mathcal{R}$ is either o-minimal or interdefinable with $(\mathbb{R},<,+, \mathcal{B}, \alpha \mathbb{Z})$ for some $\alpha>0$ and collection $\mathcal{B}$ of bounded subsets of Euclidean space such that $(\mathbb{R},<,+, \mathcal{B})$ is o-minimal.

(5) $\mathcal{R}$ is either o-minimal or locally o-minimal and interdefinable with $(\mathcal{S}, \alpha \mathbb{Z})$ for some $\alpha>0$ and o-minimal expansion $\mathcal{S}$ of $(\mathbb{R},<,+)$.

Proof. Theorem 9.3 shows that (1) implies (2). We show that (2) implies (3). Suppose that $\mathcal{R}$ is strongly dependent, noiseless, and not o-minimal. The first claim of Fact 11.6 and Theorem 2.13 together show that $\mathcal{R}$ is locally o-minimal. We show that $\mathcal{R}$ defines $\alpha \mathbb{Z}$ for some $\alpha>0$. As $\mathcal{R}$ is locally o-minimal and not o-minimal there is an infinite definable discrete subset $E$ of $\mathbb{R}$. Let $s_{1}, \ldots, s_{n}, t_{1}, \ldots, t_{n}$ be as in Fact 11.6. After possibly removing a finite subset of $E$ we suppose that $t_{i} \neq 0$ for all $i$. Let $\alpha:=\left|t_{1}\right|$. As $t_{i} / \alpha \in \mathbb{Q}$ for all $2 \leq i \leq n$ each $s_{i}+t_{i} \mathbb{N}$ is $(\mathbb{R},<,+, \alpha \mathbb{Z})$ definable. So $E$ is $(\mathbb{R},<,+, \alpha \mathbb{Z})$-definable. As $E$ is not $(\mathbb{R},<,+)$-definable, rescaling and applying Fact 11.7 shows that $(\mathbb{R},<,+, E)$ defines $\alpha \mathbb{Z}$. So $\mathcal{R}$ defines $\alpha \mathbb{Z}$.

\footnotetext{
${ }^{1}$ Thanks to John Goodrick for discussions on this point.
} 
We may now apply Fact 11.1. We get that any $\mathcal{R}$-definable subset of $\mathbb{R}$ is a finite union of sets of the form $\bigcup_{b \in B} b+A$ where $A \subseteq[0, \alpha)$ is a finite union of intervals and singletons and $B \subseteq \alpha \mathbb{Z}$ is $\mathcal{R}$-definable. So it suffices to show that an $\mathcal{R}$-definable subset $B$ of $\alpha \mathbb{Z}$ is $(\alpha \mathbb{Z},<,+)$-definable. As $B$ is discrete this follows from Fact 11.6 .

The equivalence of (3), (4), and (5) is Theorem 11.4. We show that (3) implies (1). Suppose $\mathcal{R}$ is $(\mathbb{R},<,+, \alpha \mathbb{Z})$-minimal for fixed $\alpha>0$. Then $\mathcal{R}$ is d-minimal so every definable set is constructible by Fact 2.21 so $\mathcal{R}$ is an expansion by closed sets. The remarks after Proposition 11.3 show that $\mathcal{R}$ is strongly dependent.

In the proof of Theorem 11.8 we could have applied the theorem of Dolich and Goodrick [17, Corollary 2.20] that any strongly dependent expansion of $(\mathbb{Z},<,+)$ is interdefinable with $(\mathbb{Z},<,+)$. Dolich and Goodrick's theorem on $(\mathbb{Z},<,+)$ is proven by combining that analogue of Fact 11.6 over $\mathbb{Z}$ with Fact 11.2 .

The following corollary is essentially observed in [17, Corollary 2.4]. It follows from Theorem 11.8 as a locally o-minimal expansion of $(\mathbb{R},<,+, \times)$ is o-minimal.

Theorem 11.9. Suppose $\mathcal{R}$ expands $(\mathbb{R},<,+, \times)$. Then the following are equivalent.

(1) $\mathcal{R}$ is a strongly dependent expansion by closed sets.

(2) $\mathcal{R}$ is strongly dependent and noiseless.

(3) $\mathcal{R}$ is o-minimal.

Theorem 11.8 shows that a strongly dependent expansion of $(\mathbb{R},<,+)$ by closed sets is "almost o-minimal". This is reflected in the dp-rank.

Corollary 11.10. Suppose $\mathcal{R}$ is an expansion of $(\mathbb{R},<,+)$ by closed sets. Then the dp-rank of $\mathcal{R}$ is either $\geq \aleph_{0}$ or at most two.

In contrast Dolich and Goodrick [17, Section 3.2] show that for any $n \geq 2$ there is a noisey expansion of $(\mathbb{R},<,+)$ of dp-rank $n$.

Proof. Suppose $\mathcal{R}$ has finite dp-rank. Then $\mathcal{R}$ is strongly dependent. If $\mathcal{R}$ is ominimal then $\mathcal{R}$ has dp-rank one, so suppose that $\mathcal{R}$ defines $\alpha \mathbb{Z}$ for some $\alpha>0$. Rescaling, we suppose that $\mathcal{R}$ defines $\mathbb{Z}$. As $\mathcal{R}$ defines an infinite nowhere dense subset of $\mathbb{R}$, [32, Lemma 3.3] shows that $\mathcal{R}$ has dp-rank at least two. The induced structure on $[0,1)$ is o-minimal and so has dp-rank one. The induced structure on $\mathbb{Z}$ is interdefinable with $(\mathbb{Z},<,+)$ and hence has dp-rank one. Fact 3.7 shows that $[0,1) \times \mathbb{Z}$ has dp-rank at most two. Dp-rank is preserved by definable bijections, and $[0,1) \times \mathbb{Z} \rightarrow \mathbb{R},(t, k) \mapsto t+k$ is a definable bijection, so $\mathcal{R}$ has dp-rank two.

11.3. Definable fields. The results above allow us to prove the strongly dependent case of Conjecture 5. The strongly dependent case of Conjecture 4 follows. The proof is very similar to that of Proposition 10.26 .

Lemma 11.11. Suppose $\mathcal{R}$ is a locally o-minimal expansion of $(\mathbb{R},<,+)$ which defines $\alpha \mathbb{Z}$ for some $\alpha>0$. Suppose $X$ is a nonempty definable zero-dimensional subset of $\mathbb{R}^{n}$. Then there is a definable surjection $f:(\alpha \mathbb{Z})^{m} \rightarrow X$ for some $m$.

Proof. Rescaling reduces to the case $\alpha=1$. Let $\iota_{n}$ be as defined above. Let $\mathcal{D}$ be the collection of all images of cartesian powers of $\mathbb{Z}$ under definable functions. An application of Fact 11.1 shows that $X$ is a finite union of sets of the form $\iota_{n}(A \times B)$ 
for nonempty definable $A \subseteq[0, \alpha)^{n}$ and $B \subseteq \mathbb{Z}^{n}$. It is easy to see that $\mathcal{D}$ is closed under finite unions and subsets. So we suppose $X=\iota_{n}\left(A \times \mathbb{Z}^{n}\right)$. Suppose $A$ is infinite. As the structure induced on $[0, \alpha)$ by $\mathcal{R}$ is o-minimal we have $\operatorname{dim} A \geq 1$. Fix $b \in \mathbb{Z}^{n}$. Then $A+b \subseteq X$ hence

$$
\operatorname{dim} X \geq \operatorname{dim}(A+b)=\operatorname{dim} A \geq 1 .
$$

So we may suppose that $A$ is finite. Then $X$ is a finite union of images of $\mathbb{Z}^{n}$ under functions of the form $b \mapsto \iota_{n}(a, b)$. So $X \in \mathcal{D}$.

Proposition 11.12. Suppose $\mathcal{R}$ is a strongly dependent expansion of $(\mathbb{R},<,+)$ by closed subsets of Euclidean space. Then $\mathcal{R}$ does not define an infinite zerodimensional field. If $\mathcal{R}$ interprets an infinite field then $\mathcal{R}$ is field-type.

Proof. The second claim follows from the first claim by Theorem 10.22 If $\mathcal{R}$ is o-minimal then every zero-dimensional definable set is finite so we may assume that $\mathcal{R}$ is not o-minimal. Combining Theorem 11.8, Lemma 11.11, and Proposition 11.3 it suffices to show that $(\mathbb{Z},<,+)$ does not interpret an infinite field. This is Proposition 10.25 .

We now classify strongly dependent expansions of $(\mathbb{R},<,+)$ by closed sets which are not field-type. Recall that we let $\mathbb{R}_{\mathrm{vec}}$ be the ordered vector space $(\mathbb{R},<,+,(t \mapsto$ $\left.\lambda t)_{\lambda \in \mathbb{R}}\right)$ of real numbers. We let $\mathbb{R}_{\text {bvec }}$ be the expansion of $(\mathbb{R},<,+)$ by the restriction of $t \mapsto \lambda t$ to $[0,1]$ for all $\lambda \in \mathbb{R}$. Fact 11.5 shows that $\mathbb{R}_{\text {bvec }}$ has rational scalars so $\mathbb{R}_{\text {bvec }}$ is a proper reduct of $\mathbb{R}_{\text {vec }}$.

Proposition 11.13. The following are equivalent:

(1) $\mathcal{R}$ is a strongly dependent expansion by closed sets which does not interpret an infinite field.

(2) $\mathcal{R}$ is either a reduct of $\mathbb{R}_{\mathrm{vec}}$ or of $\left(\mathbb{R}_{\mathrm{bvec}}, \alpha \mathbb{Z}\right)$ for some $\alpha>0$.

Proof. $(1) \Rightarrow(2)$ : If $\mathcal{R}$ is o-minimal then $\mathcal{R}$ is a reduct of $\mathbb{R}_{\mathrm{vec}}$ by the PeterzilStarchenko trichotomy [70. Suppose $\mathcal{R}$ is not o-minimal. Theorem 11.8 shows that $\mathcal{R}$ is interdefinable with $(\mathbb{R},<,+, \mathcal{B}, \alpha \mathbb{Z})$ for some $\alpha>0$ and collection $\mathcal{B}$ of bounded subsets of Euclidean space such that $(\mathbb{R},<,+, \mathcal{B})$ is o-minimal. As $(\mathbb{R},<,+, \mathcal{B})$ is not field-type it is a reduct of $\mathbb{R}_{\mathrm{vec}}$. It follows easily from the semilinear cell decomposition [85, Corollary 7.6] that every bounded subset of $\mathbb{R}^{m}$ definable in $\mathbb{R}_{\text {vec }}$ is already definable in $\mathbb{R}_{\text {bvec }}$. So $(\mathbb{R},<,+, \mathcal{B})$ is a reduct of $\mathbb{R}_{\text {bvec }}$.

$(2) \Rightarrow(1)$ : Note that $\mathbb{R}_{\mathrm{vec}}$ and $\left(\mathbb{R}_{\mathrm{bvec}}, \alpha \mathbb{Z}\right)$ are both expansions by closed subsets of Euclidean space. If $\mathcal{R}$ is a reduct of $\mathbb{R}_{\mathrm{vec}}$ then $\mathcal{R}$ does not interpret an infinite field by the Peterzil-Starchenko trichotomy or (see also Theorem 10.22). Fix $\alpha>0$. It suffices to show that $\left(\mathbb{R}_{\mathrm{bvec}}, \alpha \mathbb{Z}\right)$ does not interpret an infinite field. Theorem 11.4 and Proposition 11.3 together show that any bounded subset of $\mathbb{R}^{n}$ definable in

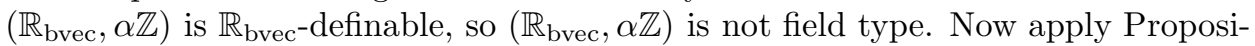
tion 11.12 .

11.4. Distal expansions. We finish this section with some observations concerning distality. (See Simon 75] for an account of distality.) Any o-minimal structure is distal and $(\mathbb{Z},<,+)$ is distal. Distality is preserved under disjoint unions and bi-interpretations. Corollary 11.14 follows. 
Corollary 11.14. Suppose $\mathcal{R}$ is an expansion of $(\mathbb{R},<,+)$ by closed sets. If $\mathcal{R}$ is strongly dependent then $\mathcal{R}$ is distal.

Every NIP expansion of $(\mathbb{R},<,+)$ by closed subsets of Euclidean space of which we are aware is distal. In particular $\left(\mathcal{S}, \lambda^{\mathbb{Z}}\right)$ is distal when $\mathcal{S}$ is an o-minimal expansion of $(\mathbb{R},<,+, \times)$ with rational exponents and $\lambda>1$, see Hieronymi and Nell [41.

Question 11.15. Is every NIP expansion of $(\mathbb{R},<,+)$ by closed sets distal?

Suppose $Z$ is a NIP expansion of $(\mathbb{Z},<,+)$. Let $(\mathbb{R},<,+, Z)$ be the expansion of $(\mathbb{R},<,+)$ by all $z$-definable subsets of $\mathbb{Z}^{n}$. It follows from Fact 2.1 that $(\mathbb{R},<,+, \mathcal{Z})$ is locally o-minimal and NIP and the structure induced on $\mathbb{Z}$ by $(\mathbb{R},<,+, Z)$ is interdefinable with $z$. So if $z$ is not distal then $(\mathbb{R},<,+, Z)$ is also not distal. Thus if there is a non-distal NIP expansion of $(\mathbb{Z},<,+)$ then there is a non-distal NIP expansion of $(\mathbb{R},<,+)$ by closed subsets of Euclidean space.

Question 11.16. Is there is a non-distal NIP expansion of $(\mathbb{N},<)$ ?

\section{Archimedean Quotients}

Through this section $\mathcal{N}$ is a highly saturated expansion of a dense ordered abelian group $(N,<,+)$. Given a positive element $a$ of $N$ we let $\mathbf{F i n}_{a}$ be the convex hull of $a \mathbb{Z}$ and $\operatorname{Inf}_{a}$ be the set of $b \in N$ such that $|n b|<a$ for all $n$. Then $\mathbf{F i n}_{a}$ and $\mathbf{I n f}_{a}$ are convex subgroups of $(N,<,+)$. We order $\mathbf{F i n}_{a} / \mathbf{I n f}_{a}$ by declaring $a+\mathbf{I n f}_{a}<b+\mathbf{I n f} \mathbf{f}_{a}$ if every element of $a+\mathbf{I n f}_{a}$ is strictly less then every element of $b+\mathbf{I n f}_{a}$. Then $\mathbf{F i n}_{a} / \mathbf{I n f} \mathbf{f}_{a}$ is an ordered abelian group. As $\mathbf{F i n}{ }_{a}$ and Inf $_{a}$ are convex Fact 3.1 shows that they are externally definable. So we consider $\mathbf{F i n}_{a} / \mathbf{I n f}_{a}$ to be an imaginary sort of $\mathcal{N}^{\mathrm{Sh}}$.

Let $\mathrm{st}_{a}: \mathbf{F i n}_{a} \rightarrow \mathbb{R}$ be given by $\operatorname{st}_{a}(b):=\sup \left\{\frac{m}{n} \in \mathbb{Q}: m a \leq n b\right\}$. Then st is $_{a}$ an ordered group homomorphism with kernal $\operatorname{Inf}_{a}$. Note that $\operatorname{st}_{a}(\alpha)=1$. Density of $(N,<,+)$ and saturation together imply that $\mathrm{st}_{a}$ is surjective. So $\mathbf{F i n}_{a} / \mathbf{I n f}_{a}$ is isomorphic to $(\mathbb{R},<,+)$.

Suppose $\mathcal{N}$ expands an ordered field $(N,<,+, \times, 0,1)$. Let $\mathbf{F i n}=\mathbf{F i n}_{1}, \mathbf{I n f}=\mathbf{I n f}_{1}$, and $s t=s_{1}$. Then st is the residue map of the archimedean valuation on $\mathcal{N}$. Let $a$ be a positive element of $N$. Then $x \mapsto a^{-1} x$ gives ordered group isomorphisms $\mathbf{F i n}_{a} \rightarrow$ Fin and $\mathbf{I n f}_{a} \rightarrow$ Inf and hence induces an isomorphism $\boldsymbol{F i n}_{a} / \mathbf{I n f}_{a} \rightarrow \mathbf{F i n} / \mathbf{I n f}$. This isomorphism is definable in $\mathcal{N}^{\text {Sh }}$. So if $\mathcal{N}$ expands an ordered field then we obtain nothing more then the residue field of the archimedean valuation.

If $\mathcal{N}$ is o-minimal then $\mathcal{N}^{\text {Sh }}$ is weakly o-minimal by the theorem of Baisalov and Poizat [1] (this also follows from Fact 3.2). In this case it easily follows that the induced structure on $\mathbf{F i n}_{a} / \mathbf{I n f} \mathbf{f}_{a}$ is o-minimal. (A weakly o-minimal expansion of $(\mathbb{R},<)$ is o-minimal.) We view Proposition 12.1 as a generalization of this fact.

Proposition 12.1. Suppose $\mathcal{N}$ is NIP and fix a positive element a of $N$. Then the structure induced on $\mathbf{F i n}_{a} / \mathbf{I n f}{ }_{a}$ by $\mathcal{N}^{\mathrm{Sh}}$ is isomorphic to a generically locally o-minimal expansion of $(\mathbb{R},<,+)$. If $\mathcal{N}$ is strongly dependent then the induced structure is either isomorphic to an o-minimal expansion of $(\mathbb{R},<,+)$ or an $(\mathbb{R},<$ $,+, \alpha \mathbb{Z})$-minimal expansion of $(\mathbb{R},<,+, \alpha \mathbb{Z})$ for some $\alpha>0$. 
Proof. For the sake of simplicity we identify $\mathbf{F i n}_{a} / \mathbf{I n f}{ }_{a}$ with $\mathbb{R}$. We show that the structure induced on $\mathbb{R}$ by $\mathcal{N}^{\mathrm{Sh}}$ is strongly noiseless. By Theorem 2.17 it suffices to show that the structure induced on $[0,1]$ by $\mathcal{N}^{\text {Sh }}$ is strongly noiseless. Let $I=[0, a]$. Note that $\operatorname{st}_{a}(I)=[0,1]$. Let $E$ be the equivalence relation of equality modulo $\operatorname{Inf}_{a}$ on $I$. Applying density we select for each $n \geq 1$ an $a_{n} \in N$ satisfying $(n-1) a_{n}<a \leq n a_{n}$. Let $F_{n}$ be the set of $(a, b) \in I^{2}$ such that $|a-b|<a_{n}$. Then each $F_{n}$ is $\mathcal{N}$-definable, $\left(F_{n}\right)_{n \in \mathbb{N}}$ is nested, and $\bigcap_{n \geq 1} F_{n}=E$. So $E$ is $\Lambda$-definable and Lemma 4.7 shows that $\left(F_{n}\right)_{n \geq 1}$ is a subdefinable basis for $F$.

By Theorem 2.17 is suffices to show that the logic topology on $[0,1]$ agrees with the usual order topology. As both topologies are compact Hausdorff it suffices to show that any subset of $[0,1]$ which is closed in the order topology is also closed in the logic topology. For this purpose it is enough to fix elements $t<t^{\prime}$ of $[0,1]$ and show that $\left[t, t^{\prime}\right]$ is closed in the logic topology. For each $m, n, m^{\prime}, n^{\prime}$ such that $\frac{m}{n} \leq t, t^{\prime} \leq \frac{m^{\prime}}{n^{\prime}}$ we let $X_{m^{\prime}, n^{\prime}}^{m, n}$ be the set of $a \in I$ such that $m \leq n a$ and $n^{\prime} a \leq m^{\prime}$. So each $X_{m^{\prime}, n^{\prime}}^{m, n}$ is $\mathcal{N}$-definable. Then st ${ }^{-1}([a, b]) \cap I$ is the intersection of all $X_{m^{\prime}, n^{\prime}}^{m, n}$ and is hence $\bigwedge$-definable. So $[a, b]$ is closed in the logic topology. The proposition now follows by Theorems 6.1 2.17, and 11.8 .

We record Proposition 12.2 for future use.

Proposition 12.2. Suppose $\mathcal{N}$ is NIP and fix a positive element a of $N$. Then the $d p$-rank of the induced structure on $\mathbf{F i n}_{a} / \mathbf{I n f} \mathbf{f}_{a}$ does not exceed the dp-rank of $\mathcal{N}$.

Proof. As $\mathbf{F i n}_{a} / \mathbf{I n f}_{a}$ is an $\mathcal{N}^{\mathrm{Sh}}$-definable image of $N$ the dp-rank of the induced structure does not exceed that of $\mathcal{N}^{\mathrm{Sh}}$. Now apply Fact 3.6

\section{Archimedean structures}

In this section we prove Theorem F, which is broken up into several theorems.

It is natural to try to extend Theorem 9.3 to expansions of archimedean ordered abelian groups. We describe counterexamples showing that the naive extension fails and then describe what we believe to be the correct extension. Recall that the classical Hahn embedding theorem states that any archimedean ordered abelian group has a unique up to rescaling embedding into $(\mathbb{R},<,+)$. So we only consider substructures of $(\mathbb{R},<,+)$. Any discrete archimedean ordered abelian group is isomorphic to $(\mathbb{Z},<,+)$, so we only consider the dense case. We suppose throughout this section that $(R,<,+)$ is an archimededan dense ordered abelian group, which we take to be a substructure of $(\mathbb{R},<,+)$, and let $\mathcal{R}$ be a structure expanding $(R,<,+)$.

13.1. Two counterexamples. Theorem 9.3 can fail for $(R,<,+)$. Recall that $(R,<,+)$, like any ordered abelian group, is NIP. Suppose $R=\mathbb{Z}+\lambda \mathbb{Z}$ for some $\lambda \in \mathbb{R} \backslash \mathbb{Q}$. Then $(R,<,+)$ is noisey as $n R$ is dense and co-dense for any $n \geq 2$.

We give a divisible counterexample. Let $\mathbb{R}_{\text {alg }}$ be the set of real algebraic numbers. Then $\left(\mathbb{R},<,+, \times, \mathbb{R}_{\text {alg }}\right)$ is NIP [34. Fix $\lambda \in \mathbb{R} \backslash \mathbb{R}_{\text {alg. }}$. Let $R:=\mathbb{R}_{\text {alg }}+\lambda \mathbb{R}_{\text {alg. Note }}$ that $(R,+)$ is divisible. Consider $(R,<,+, t \mapsto \lambda t)$, this is an NIP expansion by a continuous function. Elementary algebra yields $\lambda R=\lambda \mathbb{R}_{\text {alg }}$, so $\lambda R$ is dense and co-dense. This example is sharp in the following sense. Simon and Walsberg 77 
show that a dp-minimal expansion of a divisible archimedean ordered abelian group is weakly o-minimal. The structure induced on $\mathbb{R}_{\text {alg }}$ by $\left(\mathbb{R},<,+, \times, \mathbb{R}_{\text {alg }}\right)$ is weakly o-minimal hence dp-minimal. So by Fact $3.7(R,<,+, t \mapsto \lambda t)$ has dp-rank two.

13.2. The completion of an archimedean structure. The correct generalization of Theorem 9.3 concerns the completion of $\mathcal{R}$. This construction is motivated by work in the o-minimal and weakly o-minimal settings, which we describe below.

13.2.1. The o-minimal case. Suppose $\mathcal{R}$ is o-minimal. Laskowski and Steinhorn [54] show that there is a unique o-minimal expansion $\mathcal{S}$ of $(\mathbb{R},<,+)$ such that $\mathcal{R}$ is an elementary submodel of $\mathcal{S}$. It follows immediately that the structure induced on $R$ by $\mathcal{S}$ is a reduct of $\mathcal{R}^{\mathrm{Sh}}$. The Marker-Steinhorn theorem [60] shows that if $\mathcal{N}$ is an elementary extension of $\mathcal{S}$, and $Z \subseteq N^{n}$ is $\mathcal{N}$-definable, then $Z \cap \mathbb{R}^{n}$ is definable in $\mathcal{S}$. So any subset of $R^{n}$ externally definable in $\mathcal{R}$ is of the form $Y \cap R^{n}$ for some $\mathcal{S}$-definable $Y \subseteq \mathbb{R}^{n}$. So the structure induced on $R$ by $\mathcal{S}$ is interdefinable with $\mathcal{R}^{\mathrm{Sh}}$.

Proposition 13.1. Let $\mathcal{R}$ be o-minimal and $\mathcal{S}$ be the unique elementary extension of $\mathcal{R}$ which expands $(\mathbb{R},<,+)$. Then $\mathcal{S}$ is interdefinable with

(1) the expansion $\mathcal{S}_{1}$ of $(\mathbb{R},<,+)$ whose primitive $n$-ary relations are all sets of the form $\mathrm{Cl}(X)$ for $\mathcal{R}$-definable $X \subseteq R^{n}$, and

(2) the expansion $\mathcal{S}_{2}$ of $(\mathbb{R},<,+)$ whose primitive $n$-ary relations are all sets of the form $\mathrm{Cl}(X)$ for $\mathcal{R}^{\mathrm{Sh}}$-definable $X \subseteq R^{n}$.

We believe that (2) is the "right" definition in the general NIP setting.

Proof. Note that $\mathcal{S}_{1}$ is a reduct of $\mathcal{S}_{2}$. It follows easily from o-minimal cell decomposition that any subset of $\mathbb{R}^{n}$ which is 0 -definable in $\mathcal{S}$ is a boolean combination of closed subsets of $\mathbb{R}^{n}$ which are 0 -definable in $\mathcal{S}$. If $X \subseteq \mathbb{R}^{n}$ is closed and 0-definable in $\mathcal{S}$ then $X$ is the closure of $X^{\prime}$ in $\mathbb{R}^{n}$ where $X^{\prime}$ is the $\mathcal{R}$-definable set defined by any parameter-free formula defining $X$. So $\mathcal{S}$ is a reduct of $\mathcal{S}_{1}$.

Work of Dolich, Miller, and Steinhorn [18, Section 5] shows that $(\mathcal{S}, R)^{\circ}$ is interdefinable with $\mathcal{S}$. So if $X \subseteq R^{n}$ is definable in the structure induced on $R$ by $\mathcal{S}$ then $\mathrm{Cl}(X)$ is definable in $\mathcal{S}$. It now follows from the observations above that if $X \subseteq R^{n}$ is externally definable in $\mathcal{R}$ then $\mathrm{Cl}(X)$ is $\mathcal{S}$-definable. So $\mathcal{S}_{2}$ is a reduct of $\mathcal{S}$.

13.2.2. The weakly o-minimal case. A weakly o-minimal structure may not have weakly o-minimal theory. However, a result of Marker, Macpherson, and Steinhorn [57, Theorem 6.7] shows that a weakly o-minimal expansion of an archimedean ordered abelian group has weakly o-minimal theory, so we ignore this distinction.

Suppose $\mathcal{R}$ is weakly o-minimal. We describe the o-minimal completion of $\mathcal{R}$. This completion was first constructed by Wencel [90, 91]. The construction we use is due to Keren [52, see also [23. They define this completion in the more general setting of non-valuational structures. We specialize to the setting of archimedean structures. (Any archimedean structure is non-valuational.)

Let $\mathcal{C}(\mathcal{R})$ be set of all $t \in \mathbb{R}$ such that $(-\infty, t)$ is definable in $\mathcal{R}$. Note that $R$ is a subset of $\mathcal{C}(\mathcal{R})$ and $\mathcal{C}(\mathcal{R})$ is a subgroup of $(\mathbb{R},+)$. The completion $\overline{\mathcal{R}}$ of $\mathcal{R}$ is the expansion of $(\mathcal{C}(\mathcal{R}),<,+)$ by all closures in $\mathcal{C}(\mathcal{R})^{n}$ of $\mathcal{R}$-definable subsets of $R^{n}$. 
Then $\overline{\mathcal{R}}$ is o-minimal and the structure induced on $R$ by $\overline{\mathcal{R}}$ is interdefinable with $\mathcal{R}$.

Fact 3.2 may be applied to show that the Shelah expansion of a weakly o-minimal structure is weakly o-minimal, so $\mathcal{R}^{\mathrm{Sh}}$ is weakly o-minimal. Fact 3.1 shows that every convex subset of $R$ is definable in $\mathcal{R}^{\mathrm{Sh}}$. So $\mathcal{C}\left(\mathcal{R}^{\mathrm{Sh}}\right)=\mathbb{R}$ and $\overline{\mathcal{R}^{\mathrm{Sh}}}$ is an ominimal expansion of $(\mathbb{R},<,+)$. Note that the structure induced on $R$ by $\overline{\mathcal{R}^{\text {Sh }}}$ is interdefinable with $\mathcal{R}^{\mathrm{Sh}}$.

13.2.3. The NIP case. Generalizing what is above we define the completion of $\mathcal{R}$. Let $\mathcal{R}^{\square}$ be the structure on $\mathbb{R}$ whose primitive $n$-ary relations are all sets of the form $\mathrm{Cl}(X)$ for $\mathcal{R}^{\mathrm{Sh}}$-definable $X \subseteq R^{n}$. Note that $\mathcal{R}$ expands $(\mathbb{R},<,+)$. If $\mathcal{R}$ is o-minimal then $\mathcal{R}^{\square}$ is interdefinable with the unique elementary extension of $\mathcal{R}$ with domain $\mathbb{R}$. If $\mathcal{R}$ is weakly o-minimal then $\mathcal{R}^{\square}$ is $\overline{\mathcal{R}^{\mathrm{Sh}}}$. If $R=\mathbb{R}$ then $\mathcal{R}^{\square}$ is the open core of $\mathcal{R}^{\mathrm{Sh}}$.

Proposition 13.2 follows as $\mathrm{Cl}(X) \cap R^{n}=X$ for any $X \subseteq R^{n}$ which is closed in $R^{n}$.

Proposition 13.2. Suppose $\mathcal{R}$ is an expansion of $(R,<,+)$ by closed sets. Then $\mathcal{R}$ is a reduct of the structure induced on $R$ by $\mathcal{R}^{\square}$.

Let $\mathcal{N}$ be a highly saturated elementary extension of $\mathcal{R}$. Let Fin be the convex hull of $R$ in $N$ and let Inf be the set of $a \in N$ such that $|a|<b$ for all positive $b \in R$. Following Section 12 we identify Fin/Inf with $\mathbb{R}$ and consider $\mathbb{R}$ to be an imaginary sort of $\mathcal{N}^{\mathrm{Sh}}$. We let st $:$ Fin $\rightarrow \mathbb{R}$ be the quotient map and, abusing notation, let st : Fin ${ }^{n} \rightarrow \mathbb{R}^{n}$ be given by

$$
\operatorname{st}\left(a_{1}, \ldots, a_{n}\right)=\left(\operatorname{st}\left(a_{1}\right), \ldots, \operatorname{st}\left(a_{n}\right)\right) .
$$

Given an $\mathcal{R}$-definable subset $Y$ of $R^{n}$ we let $Y^{\prime}$ be the subset of $N^{n}$ defined by any formula which defines $Y$.

Proposition 13.3. Suppose $\mathcal{R}$ is NIP. The the following are interdefinable

(1) $\mathcal{R}^{\square}$,

(2) The expansion of $(\mathbb{R},<,+)$ by all $\operatorname{st}\left(Y \cap \mathbf{F i n}^{n}\right)$ for $\mathcal{N}$-definable $Y \subseteq N^{n}$,

(3) The open core of the structure induced on $\mathbb{R}$ by $\mathcal{N}^{\mathrm{Sh}}$.

In particular $\mathcal{R}^{\square}$ is a reduct of the structure induced on $\mathbb{R}$ by $\mathcal{N}^{\mathrm{Sh}}$. So $\mathcal{R}^{\square}$ is NIP and generically locally o-minimal and if $\mathcal{R}$ is strongly dependent then $\mathcal{R}^{\square}$ is either o-minimal or $(\mathbb{R},<,+, \alpha \mathbb{Z})$-minimal for some $\alpha>0$.

We do not know if $\mathcal{R}^{\square}$ is always interdefinable with the structure induced on $\mathbb{R}$ by $\mathcal{N}^{\text {Sh }}$. We need the next two propositions to prove the first claim of Proposition 13.3

Lemma 13.4. Suppose $X \subseteq R^{n}$ is externally definable in $\mathcal{R}$ and $Y \subseteq N^{n}$ is an honest definition of $X$. Then $\mathrm{Cl}(X)=\operatorname{st}\left(Y \cap \mathbf{F i n}^{n}\right)$.

Proof. As $X \subseteq Y \cap \mathbf{F i n}^{n}$ and st is the identity on $R^{n}$ we see that $X$ is contained in $\operatorname{st}\left(Y \cap \mathbf{F i n}^{n}\right)$. An easy saturation argument shows that $\operatorname{st}\left(Y \cap \mathbf{F i n}{ }^{n}\right)$ is closed so $\mathrm{Cl}(X)$ is contained in $\operatorname{st}\left(Y \cap \mathbf{F i n}^{n}\right)$. We prove the other inclusion. Let $I_{1}, \ldots, I_{n}$ be nonempty open intervals in $\mathbb{R}$ with endpoints in $R$. Let $U$ be $I_{1} \times \ldots \times I_{n}$. Note that the collection of such open boxes forms a basis for $\mathbb{R}^{n}$. It suffices to suppose $\mathrm{Cl}(X)$ is disjoint from $U$ and show that $\operatorname{st}\left(Y \cap \mathbf{F i n}^{n}\right)$ is disjoint from $U$. It is enough to show that $Y$ is disjoint from $\operatorname{st}^{-1}(U)$. Note that $V:=R^{n} \cap U$ is definable in $\mathcal{R}$. Then $X$ is disjoint from $V$, so $Y$ is disjoint from $V^{\prime}$ by honestness. It is easy to see that $\mathrm{st}^{-1}(U)$ is a subset of $V^{\prime}$. So $Y$ is disjoint from $\mathrm{st}^{-1}(U)$. 
Lemma 13.5. Suppose $\mathcal{R}$ is NIP. Suppose that $X$ is a closed subset of $\mathbb{R}^{n}$ which is definable in $\mathcal{N}^{\mathrm{Sh}}$. Then $X$ is definable in $\mathcal{R}^{\square}$.

Given $a=\left(a_{1}, \ldots, a_{m}\right) \in N^{m}$ we let $\|a\|=\max \left\{\left|a_{1}\right|, \ldots,\left|a_{m}\right|\right\}$. We will apply the fact that if $p, p^{\prime} \in N^{k}$ and $q, q^{\prime} \in N^{l}$ then $\left\|(p, q)-\left(p^{\prime}, q^{\prime}\right)\right\|=\max \left\{\left\|p-p^{\prime}\right\|,\left\|q-q^{\prime}\right\|\right\}$.

Proof. Let $Y:=\operatorname{st}^{-1}(X)$, so $Y$ is $\mathcal{N}^{\mathrm{Sh}}$-definable. So

$$
\left\{(\delta, a) \in N_{>0} \times N^{n}:\left\|a-a^{\prime}\right\|<\delta \quad \text { for some } a^{\prime} \in Y\right\}
$$

is $\mathcal{N}^{\mathrm{Sh}}$-definable, hence externally definable in $\mathcal{N}$. Applying Fact 3.4 we see that

$$
W:=\left\{(\delta, a) \in R_{>0} \times R^{n}:\left\|a-a^{\prime}\right\|<\delta \quad \text { for some } a^{\prime} \in Y\right\}
$$

is $\mathcal{R}^{\mathrm{Sh}}$-definable. Let $Z:=\mathrm{Cl}(W) \cap\left(\mathbb{R}_{>0} \times \mathbb{R}^{n}\right)$. So $Z$ is $\mathcal{R}^{\square}$-definable. We show that $X=\bigcap_{t>0} Z_{t}$.

To prove the left to right inclusion we fix $p \in X$ and $t \in \mathbb{R}_{>0}$ and show that $p \in Z_{t}$. As $Z_{t}$ is closed it suffices to fix $\varepsilon \in \mathbb{R}_{>0}$ and produce $p^{\prime} \in Z_{t}$ such that $\left\|p-p^{\prime}\right\|<\varepsilon$. We may suppose $\varepsilon<t$. Fix $q \in Y$ such that $\operatorname{st}(q)=p$ and $p^{\prime} \in R^{n}$ such that $\left\|p-p^{\prime}\right\|<\varepsilon$. As st $\|p-q\|=0$ we have $\left\|p^{\prime}-q\right\|<\varepsilon<t$. So $p^{\prime} \in Z_{t}$.

We prove the other inclusion. Suppose $p \in Z_{t}$ for all $t \in \mathbb{R}_{>0}$. We show $p \in X$. As $X$ is closed it suffices to fix $\varepsilon \in \mathbb{R}_{>0}$ and produce $p^{\prime} \in X$ such that $\left\|p-p^{\prime}\right\|<\varepsilon$. As $p \in Z_{\frac{1}{4} \varepsilon}$ we fix $\left(t, p^{\prime \prime}\right) \in W$ such that $\left\|\left(\frac{1}{4} \varepsilon, p\right)-\left(t, p^{\prime \prime}\right)\right\|<\frac{1}{4} \varepsilon$. So $\left|t-\frac{1}{4} \varepsilon\right|<\frac{1}{4} \varepsilon$ and $\left\|p-p^{\prime \prime}\right\|<\frac{1}{4} \varepsilon$. As $\left(t, p^{\prime \prime}\right) \in W$ there is $q \in Y$ such that $\left\|p^{\prime \prime}-q\right\|<t<\frac{1}{2} \varepsilon$. Set $p^{\prime}:=\operatorname{st}(q)$, so $p^{\prime} \in X$. As st $\left\|q-p^{\prime}\right\|=0$ we have $\left\|p^{\prime \prime}-p^{\prime}\right\|<\frac{1}{2} \varepsilon$, so $\left\|p-p^{\prime}\right\|<\varepsilon$.

We prove Proposition 13.3 As observed above it suffices to prove the first claim.

Proof. The second claim of Proposition 13.3 follows immediately from the first claim and the third claim follows from the second claim and Proposition 12.1. So we prove the first claim. Fact 3.5 and Lemma 13.4 together show that (1) is a reduct of (2). An application of saturation shows that if $Y \subseteq N^{n}$ is $\mathcal{N}$-definable then $\operatorname{st}\left(Y \cap \mathbf{F i n}^{n}\right)$ is closed. So (2) is a reduct of (3). Lemma 13.5 shows that (3) is a reduct of (1).

Corollary 13.6. If the structure induced on $\mathbb{R}$ by $\mathcal{N}^{\text {Sh }}$ is d-minimal then the induced structure is interdefinable with $\mathcal{R}^{\square}$. It follows that if $\mathcal{R}$ is strongly dependent then $\mathcal{R}^{\square}$ is interdefinable with the structure induced on $\mathbb{R}$ by $\mathcal{N}^{\text {Sh }}$.

Proof. Fact 2.21 shows that if the induced structure is d-minimal then the induced structure is interdefinable with its open core. If $\mathcal{R}$ is strongly dependent the by Proposition 12.1 the structure induced on $\mathbb{R}$ by $\mathcal{N}^{\mathrm{Sh}}$ is d-minimal.

We now investigate the structure induced on $R$ by $\mathcal{R}^{\square}$.

Proposition 13.7. Suppose $\mathcal{R}$ is NIP. Then the structure induced on $R$ by $\mathcal{R}^{\square}$ is a reduct of $\mathcal{R}^{\mathrm{Sh}}$.

Propositions 13.2 and 13.7 together show that if $\mathcal{R}$ is an NIP expansion by closed sets then the structure induced on $R$ by $\mathcal{R}^{\square}$ is between $\mathcal{R}$ and $\mathcal{R}^{\mathrm{Sh}}$.

Proof. Suppose $X$ is a subset of $\mathbb{R}^{n}$ definable in $\mathcal{R}^{\square}$. By Proposition $13.3 \mathrm{st}^{-1}(X)$ is definable in $\mathcal{N}^{\mathrm{Sh}}$, hence externally definable in $\mathcal{N}$. As st is the identity on $R^{n}$ we have $X \cap R^{n}=\mathrm{st}^{-1}(X) \cap R^{n}$. By Fact $3.4 X \cap R^{n}$ is externally definable in $\mathcal{R}$. 
If $\mathcal{R}$ is weakly o-minimal then by $13.2 .2 \mathcal{R}^{\mathrm{Sh}}$ is interdefinable with the structure induced on $R$ by $\mathcal{R}^{\mathrm{Sh}}$. The examples constructed in 42 may be adapted to show that this fails for arbitrary noisey strongly dependent expansions of archimedean ordered abelian groups.

Question 13.8. Suppose that $\mathcal{R}$ is noiseless and NIP. Must the structure induced on $R$ by $\mathcal{R}^{\square}$ be interdefinable with $\mathcal{R}^{\mathrm{Sh}}$ ?

We give a positive answer to Question 13.8 when $\mathcal{R}^{\square}$ is d-minimal. We expect natural examples of NIP expansions of $(\mathbb{R},<,+)$ by closed sets to be d-minimal, so Proposition 13.9 should cover most cases of Question 13.8 of interest.

Proposition 13.9. Suppose $\mathcal{R}$ is noiseless and NIP and suppose $\mathcal{R}^{\square}$ is d-minimal. For every $\mathcal{R}^{\mathrm{Sh}}$-definable $X \subseteq R^{n}$ there is an $\mathcal{R}^{\square}$-definable $Y \subseteq \mathbb{R}^{n}$ such that $X=$ $Y \cap R^{n}$. It follows that the structure induced on $R$ by $\mathcal{R}^{\square}$ eliminates quantifiers and is interdefinable with $\mathcal{R}^{\mathrm{Sh}}$.

The proof of Proposition 13.9 requires Lemma 13.10

Lemma 13.10. Suppose $\mathcal{R}$ is NIP. Then $\mathcal{R}$ is noiseless if and only if $\mathcal{R}$ is strongly noiseless.

Proof. Suppose $\mathcal{R}$ is not strongly noiseless. Let $X, Y$ be $\mathcal{R}$-definable subsets of $R^{n}$ such that $X$ is somewhere dense in $Y$ and has empty interior in $Y$. Let $U$ be a definable open subset of $Y$ such that $X$ is dense in $U$. After replacing $Y$ with $U$ if necessary we suppose $X$ is dense in $Y$. As $\mathcal{R}^{\square}$ is generically locally o-minimal an application of Fact 2.18 yields $0 \leq d \leq n$, a point $p \in Y$, an open neighbourhood $V$ of $p$, and a coordinate projection $\pi: \mathbb{R}^{n} \rightarrow \mathbb{R}^{d}$ such that $\pi$ gives a homeomorphism $\mathrm{Cl}(Y) \cap U \rightarrow \pi(\mathrm{Cl}(Y) \cap U)$ and $\pi(\mathrm{Cl}(Y) \cap U)$ is an open subset of $\mathbb{R}^{d}$. Then $\pi(X \cap U)$ is dense and co-dense in an open subset of $R^{d}$. So $\mathcal{R}$ is noisey.

We now prove Proposition 13.9. The proof makes use of the Pillay rank defined in Section 2.4. We let $\mathrm{Cl}_{R}(X)$ be the closure in $R^{n}$ of $X \subseteq R^{n}$.

Proof. The second claim follows from the first claim by Proposition 13.7, so we only prove the first claim. Lemma 13.10 shows that $\mathcal{R}$ is strongly noiseless. Proposition 5.4 shows that $\mathcal{R}^{\text {Sh }}$ is strongly noiseless.

Let $X$ be an $\mathcal{R}^{\text {Sh }}$-definable subset of $R^{n}$. We apply induction on the Pillay rank of $\mathrm{Cl}(X)$. If $\operatorname{Pr}(\mathrm{Cl}(X))=-1$ then $X$ is empty, so we take $Y=\emptyset$. Suppose $\operatorname{Pr}(\mathrm{Cl}(X)) \geq 0$. Let $U$ be the interior of $X$ in $\mathrm{Cl}_{R}(X)$ and $Z:=\mathrm{Cl}_{R}(X) \backslash U$. Then $U, Z$ are $\mathcal{R}^{\mathrm{Sh}}$-definable and $Z$ is closed in $R^{n}$. By strong noiselessness $U$ is dense in $\mathrm{Cl}_{R}(X)$ so $Z$ is nowhere dense in $\mathrm{Cl}_{R}(X)$ hence $\mathrm{Cl}(Z)$ is nowhere dense in $\mathrm{Cl}(X)$. So $\operatorname{Pr}(\mathrm{Cl}(Z))<\operatorname{Pr}(\mathrm{Cl}(X))$. Proposition 13.7 shows that $Z \cap X$ is definable in $\mathcal{R}^{\mathrm{Sh}}$ so an application of induction shows that $Z \cap X=Y^{\prime} \cap R^{n}$ for some $\mathcal{R}^{\square}$-definable $Y^{\prime} \subseteq \mathbb{R}^{n}$. We have $X \backslash Z=U$ and $U=[\mathrm{Cl}(X) \backslash \mathrm{Cl}(Z)] \cap R^{n}$. So

$$
X=\left[Y^{\prime} \cup(\mathrm{Cl}(X) \backslash \mathrm{Cl}(Z))\right] \cap R^{n}
$$

and

is $\mathcal{R}^{\square}$-definable.

$$
Y:=Y^{\prime} \cup(\mathrm{Cl}(X) \backslash \mathrm{Cl}(Z))
$$

Proposition 13.3 shows that if $\mathcal{R}$ is strongly dependent then $\mathcal{R}^{\square}$ is d-minimal. So Proposition 11.12 follows from Proposition 13.9 . 
Proposition 13.11. Suppose $\mathcal{R}$ is strongly dependent and noiseless. For every $\mathcal{R}^{\mathrm{Sh}}$-definable $X \subseteq R^{n}$ there is an $\mathcal{R}^{\square}$-definable $Y \subseteq \mathbb{R}^{n}$ such that $X=Y \cap R^{n}$. In follows that the structure induced on $R$ by $\mathcal{R}^{\square}$ eliminates quantifiers and is interdefinable with $\mathcal{R}^{\mathrm{Sh}}$.

Proposition 13.11 shows that if $\mathcal{R}$ is strongly dependent and noiseless then $\mathcal{R}$ is "locally weakly o-minimal". One example of such a structure is the structure induced on $\mathbb{Q}$ by $(\mathbb{R},<,+, \mathbb{Z}, \mathbb{Q})$, which is strongly dependent by [17, Proposition 3.1].

We show how the weakly o-minimal case may be recovered from the general case. Suppose $\mathcal{R}$ is weakly o-minimal. Then $\mathcal{N}$ is weakly o-minimal and so $\mathcal{N}^{\mathrm{Sh}}$ is weakly o-minimal by Fact 3.2. It easily follows that the structure induced on $\mathbb{R}$ by $\mathcal{N}^{\mathrm{Sh}}$ is weakly o-minimal, hence o-minimal. So $\mathcal{R}^{\square}$ is o-minimal. The weak o-minimal cell decomposition [57, Theorem 4.11] shows that a weakly o-minimal structure is noiseless. So the structure induced on $\mathbb{R}$ by $\mathcal{R}^{\square}$ is interdefinable with $\mathcal{R}^{\mathrm{Sh}}$.

As an application we describe the dp-ranks of noiseless expansions of archimedean ordered abelian groups. Corollary 13.12 generalizes Corollary 11.10 ,

Corollary 13.12. Suppose $\mathcal{R}$ is noiseless. Then the dp-rank of $\mathcal{R}$ is either $\geq \aleph_{0}$ or at most two. If $\mathcal{R}$ is strongly dependent then the dp-rank of $\mathcal{R}$ agrees with that of $\mathcal{R}^{\square}$.

We will apply Proposition 13.13, Onshuus and Usvyatsov's proof of Fact 3.6 immediately yields Proposition 13.13 , we leave the details to the reader.

Proposition 13.13. Let $\mathcal{M}$ be an NIP structure. Suppose $A$ is a subset of $M$ and suppose that the structure induced on $A$ by $\mathcal{M}$ eliminates quantifiers. Then the $d p$-rank of the induced structure on $A$ is no greater than the dp-rank of $\mathcal{M}$.

We now prove Corollary 13.12

Proof. Suppose $\mathcal{R}$ has finite dp-rank. Then $\mathcal{R}$ is strongly dependent. Propositions 13.11 and 13.13 together show that the dp-rank of $\mathcal{R}$ does not exceed that of $\mathcal{R}^{\square}$. Combining Proposition 13.3 with Proposition 12.2 we see that the dp-rank of $\mathcal{R}^{\square}$ does not exceed that of $\mathcal{R}$. Proposition 13.3 and Corollary 11.10 together show that $\mathcal{R}^{\square}$ has dp-rank at most two.

Proposition 13.3. Proposition 13.11, and Corollary 13.12 together show that $\mathcal{R}$ is noiseless and dp-minimal if and only if $\mathcal{R}$ is weakly o-minimal. This also follows from [77.

It now seems natural to study the "dense-pair" $\left(\mathcal{R}^{\square}, \mathcal{R}\right)$. Hieronymi and Günaydin 34 show that $\left(\mathcal{R}^{\square}, \mathcal{R}\right)$ is NIP when $\mathcal{R}$ is o-minimal. Bar-Yehuda, Hasson, and Peterzil 22] (combined with [13, Corollary 2.5]) shows that $(\overline{\mathcal{R}}, \mathcal{R})$ is NIP when $\mathcal{R}$ is weakly o-minimal, it follows that $\left(\mathcal{R}^{\square}, \mathcal{R}\right)$ is NIP when $\mathcal{R}$ is weakly o-minimal. Conjecture 8 will probably require more sophisticated tools then are currently available.

Conjecture 8. If $\mathcal{R}$ is NIP then $\left(\mathcal{R}^{\square}, \mathcal{R}\right)$ is NIP.

Conjecture 8 would reduce the study of NIP expansions of dense archimedean ordered groups to the study of NIP expansions of $(\mathbb{R},<,+)$. 
13.3. Basic examples. Finally, we discuss a few basic examples. It will be shown in 88 , that if $R$ is a dense subgroup of $(\mathbb{R},<,+)$ then $(R,<,+)^{\square}$ is interdefinable with $(\mathbb{R},<,+)$ and $(R,<,+)^{\text {Sh }}$ is interdefinable with the structure induced on $R$ by $(\mathbb{R},<,+)$. So the completion of a dense archimedean ordered abelian group in our sense agrees with the usual notion of completion.

Suppose that $R$ is an NIP subfield of $(\mathbb{R},<,+, \times)$. One hopes that $(R,<,+, \times)^{\square}$ agrees with the usual completion, i.e. is interdefinable with $(\mathbb{R},<,+, \times)$. We show that this follows from the main conjecture on NIP fields.

It is a well-known conjecture that an infinite NIP field is either separably closed, real closed, or admits a non-trivial Henselian valuation (see for example [35]). An ordered field cannot be separably closed, a Henselian valuation on an ordered field has a convex valuation ring [25, Lemma 4.3.6], and an archimedean ordered field does not admit a non-trivial convex subring. So the conjecture implies that an NIP archimedean ordered field is real closed. Suppose $R$ is real closed. Then $(R,<,+, \times)$ is o-minimal and $(\mathbb{R},<,+, \times)$ is an elementary extension of $(R,<,+, \times)$. So it follows from Section 13.2 .1 that $(R,<,+, \times)^{\square}$ is interdefinable with $(\mathbb{R},<,+, \times)$ and $(R,<,+, \times)^{\mathrm{Sh}}$ is interdefinable with the structure induced on $R$ by $(\mathbb{R},<,+, \times)$.

\section{Definable groups}

In this section we presume the reader is familiar with the theory of definable groups in NIP structures and basic facts about definable groups in o-minimal structures. Our reference for definable groups in NIP structures is [75]. Throughout this section $\mathcal{N}$ is a highly saturated NIP structure, $G$ is an $\mathcal{N}$-definable group, and $\pi$ is the quotient $\operatorname{map} G \rightarrow G / G^{00}$.

Fact 14.1 follows from the proof of [46, Theorem 10.1]. Fact 14.1]depends on a long line of work on definable groups in o-minimal structures due to may authors.

Fact 14.1. Suppose $\mathcal{N}$ is an o-minimal expansion of an ordered field and $G$ is definably compact. Then

(1) $G^{00}$ is externally definable in $\mathcal{N}$,

(2) $G$ is compactly dominated by $\pi$, and

(3) $G / G^{00}$ is "semi-o-minimal", i.e., $G / G^{00}$ is definable in a disjoint union of finitely many o-minimal expansions of $(\mathbb{R},<)$, each of which is interpretable in $\mathcal{N}^{\mathrm{Sh}}$.

It is natural to ask if there are more general assumptions under which the conclusions of Fact 14.1 hold. There are satisfactory generalizations of (1) and (2). Fact 14.2. proven by Hrushovski and Pillay [47, Lemma 8.2, Remark 8.3], generalizes (1). Recall that a definably compact group in an o-minimal structure is definably amenable.

Fact 14.2. Suppose that $G$ is definably amenable and $G / G^{00}$ is a Lie group. Then $G^{00}$ is definable in $\mathcal{N}^{\text {Sh }}$.

Recall that $G / G^{00}$ is a Lie group if and only if there is a subgroup $H$ of $G$ definable in $\mathcal{N}$ such that $H$ has finite index in $G$ and $H$ has no $\mathcal{N}$-definable subgroups of finite index. If such $H$ exists then we view $H$ as a "definably connected component of the 
identity". Note also that $G / G^{00}$ is a connected Lie group if and only if $G$ has no $\mathcal{N}$-definable subgroups of finite index, a condition we view as a form of "definable connectedness".

Fact 14.3 generalizes (2). Fact 14.3 follows from the measure-theoretic definition of distality [75, Proposition 9.26] and [75, Proposition 8.32, Theorem 8.37, Lemma 8.36]. Recall that an o-minimal structure is distal and a definable group in an o-minimal structure is fsg if and only if it is definably compact.

Fact 14.3. If $\mathcal{N}$ is distal and $G$ is $f$ sg then $G$ is compactly dominated by $\pi$.

The question remains if we can generalize (3). At a minimum one must assume $G / G^{00}$ is a Lie group. It is also natural to assume that $G$ is definably amenable as to ensure $G^{00}$ is externally definable.

Question 14.4. If $\mathcal{N}$ is strongly dependent and $G$ is definably amenable then must $G / G^{00}$ be definable in a disjoint union of finitely many o-minimal expansions of $(\mathbb{R},<)$, each of which is interpretable in $\mathcal{N}^{\mathrm{Sh}}$ ?

Suppose Question 14.4 admits an affirmative answer. It follows that if $\mathcal{N}$ is distal and strongly dependant, $G$ is fsg, and $G$ has no $\mathcal{N}$-definable subgroups of finite index, then $G^{00}$ is definable in $\mathcal{N}^{\mathrm{Sh}}, G$ is compactly dominated by $\pi$, and $G / G^{00}$ is semi-o-minimal. Informally: if $\mathcal{N}$ satisfies two key abstract properties of o-minimal structures, $G$ is "definably compact" and "definably connected", then $\mathcal{N}^{\text {Sh }}$ defines an "intrinsic standard part map" from $G$ to a compact connected Lie group $G / G^{00}$, the structure on $G$ is closely related to the induced structure on $G / G^{00}$, and the induced structure on $G / G^{00}$ is "semi-o-minimal".

We describe an example which should witness the necessity of strong dependence. Let $\mathcal{S}$ be an o-minimal expansion of $(\mathbb{R},<,+, \times)$ with rational exponents. Suppose $\mathcal{N}$ is a highly saturated elementary expansion of $\left(\mathcal{S}, \lambda^{\mathbb{Z}}\right)$ for some $\lambda>1$. Let $G$ be the unit circle in $\mathcal{N}$ equipped with complex multiplication. We expect that $G$ is fsg hence definably amenable. We further expect that $G / G^{00}$ can be identified with the unit circle in $\mathbb{C}$ and that the structure induced on $G / G^{00}$ by $\mathcal{N}^{\text {Sh }}$ is interdefinable with the structure induced on the unit circle by $\left(\mathcal{S}, \lambda^{\mathbb{Z}}\right)$. So in this case $G / G^{00}$ should not be semi-o-minimal.

Theorem 14.5 handles the case when $G / G^{00}$ is a simple centerless Lie group. Suppose $H$ is a Lie group. Then $H$ is said to be simple if every closed normal subgroup of $H$ is discrete. Recall that any discrete subgroup of $H$ is contained in the center of $H$ and the center of $H$ is a normal subgroup. So $H$ is simple and centerless if and only if $H$ has no non-trivial closed normal subgroups.

Theorem 14.5. Suppose $G$ is definably amenable and $G / G^{00}$ is a simple centerless Lie group. Then the structure induced on $G / G^{00}$ by $\mathcal{N}^{\text {Sh }}$ is bi-interpretable with a generically locally o-minimal expansion of $(\mathbb{R},<,+, \times)$. If $\mathcal{N}$ is in addition strongly dependent then the induced structure on $G / G^{00}$ is bi-interpretable with an o-minimal expansion of $(\mathbb{R},<,+, \times)$.

Let $H$ be a simple centerless compact Lie group. Nesin and Pillay [67] show that $H$ (as an abstract group) is bi-interpretable with $(\mathbb{R},<,+, \times)$. It follows from their proof that $H$ (an as abstract group) defines a basis for the group topology on $H$. 
So we do not distinguish between $H$ as an abstract group and $H$ as a Lie group. (This idea is essentially already present in classical Lie theory.)

Theorem 14.5 is proven in the following way. Applying 67 we see that the induced structure on $G / G^{00}$ is bi-interpretable with an expansion $\mathcal{R}$ of $(\mathbb{R},<,+, \times)$. Theorem 6.1 shows $\mathcal{R}$ is strongly noiseless, hence generically locally o-minimal. Theorem 11.9 (essentially due to Dolich and Goodrick) shows that $\mathcal{R}$ is o-minimal when $\mathcal{N}$ is strongly dependent.

Proposition 14.6. Suppose $H$ is a simple centerless compact Lie group. Suppose $\mathcal{H}$ is a strongly noiseless expansion of $H$. Then $\mathcal{H}$ is bi-interpretable with a generically locally o-minimal expansion of $(\mathbb{R},<,+, \times)$. If $\mathcal{H}$ is in addition strongly dependent then $\mathcal{H}$ is bi-interpretable with an o-minimal expansion of $(\mathbb{R},<,+, \times)$.

Proof. Note first that $\mathcal{H}$ is bi-interpretable with an expansion of $(\mathbb{R},<,+, \times)$ as $H$ is bi-interpretable with $(\mathbb{R},<,+, \times)$. We therefore regard $\mathbb{R}$ as an imaginary sort of $\mathcal{H}$ and show that the structure induced on $\mathbb{R}$ by $\mathcal{H}$ is generically locally o-minimal. By [67, Theorem 0.1, Corollary 2.3] there is a subgroup $G$ of $\mathrm{Gl}_{n}(\mathbb{R})$ and an $\mathcal{H}-$ definable continuous group isomorphism $u: G \rightarrow H$. O-minimal cell decomposition is easily applied to obtain a semialgebraic homeomorphic embedding $v: \mathbb{R} \rightarrow G$. So $u \circ v$ is an $\mathcal{H}$-definable homeomorphic embedding $\mathbb{R} \rightarrow H$. It follows that the induced structure on $\mathbb{R}$ is strongly noiseless, hence generically locally o-minimal by Theorem 2.17.

If $\mathcal{H}$ is strongly dependent then the structure induced on $\mathbb{R}$ by $\mathcal{H}$ is strongly dependent and noiseless, hence o-minimal by Theorem 11.9.

We can now prove Theorem 14.5 .

Proof. Fact 14.2 shows that $G^{00}$ is definable in $\mathcal{N}^{\text {Sh }}$. As the group topology on $G / G^{00}$ is definable in $\mathcal{N}^{\mathrm{Sh}}$, Theorem 6.1 shows that the structure induced on $G / G^{00}$ is strongly noiseless. An application of Proposition 14.6 now shows that the structure induced on $G / G^{00}$ by $\mathcal{N}^{\mathrm{Sh}}$ is bi-interpretable with a generically locally o-minimal expansion of $(\mathbb{R},<,+, \times)$ in general and bi-interpretable with an o-minimal expansion of $(\mathbb{R},<,+, \times)$ when $\mathcal{N}$ is strongly dependent.

Question 14.4 naturally splits into two questions. Does $\mathcal{N}^{\text {Sh }}$ define a basis for the logic topology on $G / G^{00}$ ? If $H$ is a compact Lie group (considered as an abstract group), $\mathcal{H}$ is a first order expansion of $H$ which defines a basis for the group topology on $H$, and $\mathcal{H}$ is strongly dependent and strongly noiseless, then must $\mathcal{H}$ be interpretable in a finite disjoint union of o-minimal expansions of $(\mathbb{R},<)$ ?

\section{Modular SPECUlations}

It is a known open question to define a good notion of "modularity" or "onebasedness" for NIP structures. The Peterzil-Starchenko trichotomy [70] seems to show that an o-minimal structure is "modular" if and only if it does not define an infinite field. In particular an o-minimal expansion $\mathcal{R}$ of $(\mathbb{R},<,+)$ satisfies exactly one of the following

(1) $\mathcal{R}$ defines an infinite field,

(2) $\mathcal{R}$ is a reduct of $\mathbb{R}_{\mathrm{vec}}$. 
So an o-minimal structure should be modular if and only if (2) holds. It seems likely that one might arrive at the right general definition by proving similar dichotomies for more general classes of structures. We discuss the case of weakly o-minimal expansions of archimedean ordered abelian groups, more generally noiseless strongly dependent expansions of archimedean densely ordered abelian groups. We make use of the notation and results of Section 13.2 .

We believe that a good notion of modularity for NIP structures should satisfy the following:

(A1) A modular structure cannot interpret an infinite field.

(A2) Any ordered abelian group and any ordered vector space is modular.

(A3) Modularity is preserved under disjoint unions and bi-interpretations.

(A4) Suppose $\mathcal{M}$ is a modular NIP structure, $A$ is a subset of $M^{x}$, and the structure induced on $A$ by $\mathcal{M}$ eliminates quantifiers. Then the induced structure on $A$ is modular. (So in particular the Shelah expansion of a modular NIP structure is modular.)

(A1) is a well-known property of one-based stable structures, see for example [48. We do not know if (A4) has been observed in the literature for one-based stable structures, so we briefly indicate why it is true. A formula $\phi(x, y)$ is weakly normal if there is $n$ such that whenever $a_{1}, \ldots, a_{n} \in M^{x}$ satisfy $\bigcap_{i=1}^{n} \phi\left(a_{i}, M^{y}\right) \neq \emptyset$ then $\phi\left(a_{i}, M^{y}\right)=\phi\left(a_{j}, M^{y}\right)$ for some $1 \leq i<j \leq n$. The structure $\mathcal{M}$ is said to be weakly normal if every formula is a boolean combination of weakly normal formulas. Hrushovski and Pillay show that $\mathcal{M}$ is weakly normal if and only if $\mathcal{M}$ is stable and one-based [48. It is easy to see that if $\mathcal{M}$ is weakly normal, $A$ is a subset of $M^{x}$, and the structure induced on $A$ by $\mathcal{M}$ eliminates quantifiers then the induced structure is weakly normal.

Following Section 13.2 we let $(R,<,+)$ be a dense archimedean ordered abelian group, which we take to be a substructure of $(\mathbb{R},<,+)$, let $\mathcal{R}$ be an expansion of $(R,<,+)$, and let $\mathcal{N}$ be a highly saturated elementary extension of $\mathcal{R}$.

Proposition 15.1. Suppose $\mathcal{R}$ is strongly dependent and noiseless. Then one of the following holds.

(1) $\mathcal{N}^{\mathrm{Sh}}$ interprets $(\mathbb{R},<,+, \times)$,

(2) every $\mathcal{R}$-definable subset of $R^{n}$ is of the form $X \cap \mathbb{R}^{n}$ for some $\mathbb{R}_{\mathrm{vec}}$-definable subset $X$ of $\mathbb{R}^{n}$,

(3) there is an $\alpha>0$ such that every $\mathcal{R}$-definable subset of $R^{n}$ is of the form $X \cap R^{n}$ for some $\left(\mathbb{R}_{\mathrm{bvec}}, \alpha \mathbb{Z}\right)$-definable subset $X$ of $\mathbb{R}^{n}$.

Proof. Recall that $\mathcal{R}^{\square}$ is interpretable in $\mathcal{N}^{\text {Sh }}$, so (1) holds when $\mathcal{R}^{\square}$ is field-type. Suppose $\mathcal{R}^{\square}$ is not field-type. Proposition 13.3 and Proposition 11.13 together show that $\mathcal{R}^{\square}$ is either a reduct of $\mathbb{R}_{\text {vec }}$ or $\mathbb{R}_{\text {bvec }}$. Proposition 13.11 shows that if $\mathcal{R}^{\square}$ is a reduct of $\mathbb{R}_{\text {vec }}$ then (2) holds and if $\mathcal{R}^{\square}$ is a reduct of $\left(\mathbb{R}_{\text {bvec }}, \alpha \mathbb{Z}\right)$ then (3) holds.

Suppose for the moment that we have a notion of modularity satisfying (A1)(A4). Fix $\alpha>0$ and let $\mathcal{J}$ be the structure induced on $[0, \alpha)$ by $\mathbb{R}_{\mathrm{vec}}$. (A2) and (A4) together imply that $\mathcal{J}$ is modular. As $\left(\mathbb{R}_{\text {bvec }}, \alpha \mathbb{Z}\right)$ is bi-interpretable with the disjoint union of $\mathcal{J}$ and $(\mathbb{Z},<,+),(\mathrm{A} 2)$ and (A3) together imply that $\left(\mathbb{R}_{\text {bvec }}, \alpha \mathbb{Z}\right)$ is modular. So if $\mathcal{R}$ satisfies (2) or (3) of Proposition 15.1 then (A4) implies $\mathcal{R}$ is modular. 
Conjecture 9. Suppose $\mathcal{R}$ is strongly dependent and noiseless. If $\mathcal{R}^{\square}$ is not fieldtype then $\mathcal{N}^{\mathrm{Sh}}$ does not interpret an infinite field.

If Conjecture 9 fails then (A1) - (A4) are inconsistent. It is presumably possible to give a "brute-force" proof. The "right" solution would be to define a notion of modularity satisfying (A1) - (A4).

Block Gorman, Hieronymi, and Kaplan [33, Section 5.2] study $\left(\mathbb{R}_{\text {vec }}, \mathbb{Q}\right)$. It follows from their work that $\left(\mathbb{R}_{\mathrm{vec}}, \mathbb{Q}\right)$ is NIP, the induced structure on $\mathbb{Q}$ is weakly o-minimal, and $\left(\mathbb{R}_{\mathrm{vec}}, \mathbb{Q}\right)^{\circ}$ is interdefinable with $\mathbb{R}_{\mathrm{vec}}$. We expect the structure induced on $\mathbb{Q}$ by $\left(\mathbb{R}_{\mathrm{vec}}, \mathbb{Q}\right)$ to be a typical example of a modular weakly o-minimal structure.

We now give an example of a weakly o-minimal structure which does not interpret an infinite field, but whose Shelah expansion interprets $(\mathbb{R},<,+, \times)$. This example also shows that modularity cannot be defined in terms of algebraic closure.

We first recall the Mann property. We refer to Günaydin and van den Dries [86] for more information and references. Let $\mathbb{R}_{>0}$ be the set of positive real numbers. A solution $\left(s_{1}, \ldots, s_{n}\right)$ of the equation $a_{1} x_{1}+\ldots a_{n} x_{n}=1$ is non-degenerate if $\sum_{i \in I} a_{i} s_{i} \neq 0$ for all $I \subseteq\{1, \ldots, n\}$. A subgroup $G$ of $\mathbb{C}^{\times}$has the Mann property if for any $a_{1}, \ldots, a_{n} \in \mathbb{C}^{\times}$the equation $a_{1} x_{1}+\ldots+a_{n} x_{n}=1$ has only finitely many non-degenerate solutions in $G^{n}$. It is a deep diophantine result that any finite rank subgroup of $\mathbb{C}^{\times}$has the Mann property. In particular the group of complex roots of unity has the Mann property and if $a_{1}, \ldots, a_{m} \in \mathbb{C}^{\times}$then $\left\{a_{1}^{q_{1}} \ldots a_{m}^{q_{m}}: q_{1}, \ldots, q_{m} \in \mathbb{Q}\right\}$ has the Mann property. If $G$ is a dense subgroup of $\left(\mathbb{R}_{>0}, \times\right)$ with the Mann property then $(\mathbb{R},<,+, \times, G)$ is NIP.

Fix a divisible subgroup $G$ of $\left(\mathbb{R}_{>0}, \times\right)$ with the Mann property. Note that $(G,<, \times)$ is archimedean. Let $\mathcal{G}$ be the structure induced on $G$ by $(\mathbb{R},<,+, \times, G)$. By [86, Theorem 7.2] any $\mathcal{G}$-definable subset of $G^{n}$ is of the form $Y \cap G^{n}$ for semialgebraic $Y \subseteq \mathbb{R}^{n}$ (this requires divisibility). In particular $\mathcal{G}$ is weakly o-minimal and the structure induced on $G$ by $(\mathbb{R},<,+, \times)$ eliminates quantifiers. Given polynomials $f_{1}, \ldots, f_{m} \in \mathbb{R}\left[x_{1}, \ldots, x_{n}\right]$ it follows from [86, Proposition 5.8] that

$$
\left\{g \in G^{n}: f_{1}(g)=\ldots=f_{m}(g)=0\right\}
$$

is a finite union of cosets of subgroups of $G^{n}$ of the form

$$
\left\{\left(g_{1}, \ldots, g_{n}\right) \in G^{n}: g_{1}^{i_{1}} \ldots g_{n}^{i_{n}}=1\right\}
$$

for integer $i_{1}, \ldots, i_{n}$ (they only prove this for algebraically closed fields, but the same proof goes through over $\mathbb{R}$ ). A semialgebraic subset of $\mathbb{R}^{n}$ has empty interior if and only if its Zariski closure has empty interior (see for example [7, 2.8]), it follows that any $\mathcal{G}$-definable subset of $G^{n}$ either has interior or is contained in a nowhere dense $(G, \times)$-definable subset of $G^{n}$. It is now easy to see that algebraic closure in $\mathcal{G}$ agrees with algebraic closure in $(G, \times)$.

Proposition 15.2. Let $\mathcal{H}$ be a highly saturated elementary extension of $\mathcal{G}$. Then $\mathcal{H}$ does not interpret an infinite field but $\mathcal{H}^{\mathrm{Sh}}$ interprets $(\mathbb{R},<,+, \times)$.

A similar argument shows that if $U$ is the set of complex roots of unity, $U$ is the structure induced on $U$ by $(\mathbb{R},<,+, \times, U)$, and $\mathcal{V}$ is a highly saturated elementary 
expansion of $\mathcal{U}$, then $\mathcal{V}$ does not interpret an infinite field but $\mathcal{V}^{\text {Sh }}$ interprets $(\mathbb{R},<$ $,+, \times)$. We let $\mathrm{Cl}(X)$ be the closure in $\left(\mathbb{R}_{>0}\right)^{n}$ of $X \subseteq\left(\mathbb{R}_{>0}\right)^{n}$. Recall that an open set $U$ is regular if $U$ agrees with the interior of the closure of $U$.

Proof. Eleftheriou 22 shows that $\mathcal{G}$, and hence $\mathcal{H}$, eliminates imaginaries. So it suffices to show that $\mathcal{H}$ does not define an infinite field. This may be deduced from the observations on algebraic closure above. It also follows from Berenstein and Vassiliev [5. Proposition 2.11, Proposition 3.16]. (It is easy to see that a structure which defines an infinite field defines a partial almost quasidesign in the sense of [5].)

We show that $\mathcal{H}^{\mathrm{Sh}}$ interprets $(\mathbb{R},<,+, \times)$. Let $\mathcal{G}^{\square}$ be the expansion of $\left(\mathbb{R}_{>0},<, \times\right)$ by $\mathrm{Cl}(X)$ for every $\mathcal{G}^{\mathrm{Sh}}$-definable $X \subseteq G^{n}$. The arguments of Section 13.2 show that $\mathcal{G}^{\square}$ is interpretable in $\mathcal{H}^{\mathrm{Sh}}$. We show that $\mathcal{G}^{\square}$ defines $(\mathbb{R},<,+, \times)$. It suffices to show that

$$
\left\{\left(t, t^{\prime}, s\right) \in\left(\mathbb{R}_{>0}\right)^{3}: t t^{\prime}<s\right\} \text { and }\left\{\left(t, t^{\prime}, s\right) \in\left(\mathbb{R}_{>0}\right)^{3}: t+t^{\prime}<s\right\}
$$

are both definable in $\mathcal{G}^{\square}$. Both of these are regular open subsets of $\left(\mathbb{R}_{>0}\right)^{3}$. So let $U$ be any regular open semialgebraic subset of $\left(\mathbb{R}_{>0}\right)^{n}$. Then $U \cap G^{n}$ is $\mathcal{G}$-definable so $\mathrm{Cl}\left(U \cap G^{n}\right)$ is $\mathcal{G}^{\square}$-definable. As $U$ is open and $U \cap G^{n}$ is dense in $U$ we have $\mathrm{Cl}\left(U \cap G^{n}\right)=\mathrm{Cl}(U)$. So $U$ is $\mathcal{G}^{\square}$-definable as it is the interior of $\mathrm{Cl}(U)$.

\section{REFERENCES}

[1] Y. Baisalov and B. Poizat. Paires de structures o-minimales. J. Symbolic Logic, 63(2):570-578, 1998.

[2] E. Bar-Yehuda, A. Hasson, and Y. Peterzil. A theory of pairs for non-valuational structures. J. Symb. Log., 84(2):664-683, 2019.

[3] L. Bélair. Panorama of p-adic model theory. Ann. Sci. Math. Québec, 36(1):43-75 (2013), 2012.

[4] O. Belegradek, V. Verbovskiy, and F. O. Wagner. Coset-minimal groups. Ann. Pure Appl. Logic, 121(2-3):113-143, 2003.

[5] A. Berenstein and E. Vassiliev. Weakly one-based geometric theories. J. Symbolic Logic, $77(2): 392-422,2012$.

[6] A. Bès and C. Choffrut. Theories of real addition with and without a predicate for integers. Preprint, arXiv:2002.04282, 2020.

[7] J. Bochnak, M. Coste, and M.-F. Roy. Real Algebraic Geometry. Springer, 1998.

[8] B. Boigelot, J. Brusten, and J. Leroux. A generalization of semenov's theorem to automata over real numbers. In Automated Deduction - CADE-22, pages 469-484. Springer Berlin Heidelberg, 2009.

[9] B. Boigelot, S. Rassart, and P. Wolper. On the expressiveness of real and integer arithmetic automata (extended abstract). In Proceedings of the 25th International Colloquium on Automata, Languages and Programming, ICALP '98, pages 152-163, London, UK, UK, 1998. Springer-Verlag.

[10] F. Bouchy, A. Finkel, and J. Leroux. Decomposition of decidable first-order logics over integers and reals. In 2008 15th International Symposium on Temporal Representation and Reasoning. IEEE, June 2008.

[11] J. R. Büchi. On a decision method in restricted second order arithmetic. In Logic, Methodology and Philosophy of Science (Proc. 1960 Internat. Congr .), pages 1-11. Stanford Univ. Press, Stanford, Calif., 1962.

[12] S. Chambille, P. Cubides Kovacsics, and E. Leenknegt. Clustered cell decomposition in Pminimal structures. Ann. Pure Appl. Logic, 168(11):2050-2086, 2017.

[13] A. Chernikov and P. Simon. Externally definable sets and dependent pairs. Israel J. Math., 194(1):409-425, 2013.

[14] A. Chernikov and P. Simon. Externally definable sets and dependent pairs II. Trans. Amer. Math. Soc., 367(7):5217-5235, 2015. 
[15] F. Delon. Q muni de l'arithmétique faible de Penzin est décidable. Proc. Amer. Math. Soc., 125(9):2711-2717, 1997.

[16] A. Dolich and J. Goodrick. Tame topology over definable uniform structures: viscerality and dp-minimality. arXiv:1505.06455, 2015.

[17] A. Dolich and J. Goodrick. Strong theories of ordered Abelian groups. Fund. Math., 236(3):269-296, 2017.

[18] A. Dolich, C. Miller, and C. Steinhorn. Structures having o-minimal open core. Trans. Amer. Math. Soc., 362(3):1371-1411, 2010.

[19] R. Dougherty and C. Miller. Definable Boolean combinations of open sets are Boolean combinations of open definable sets. Illinois J. Math., 45(4):1347-1350, 2001.

[20] O. Dovgoshey, O. Martio, V. Ryazanov, and M. Vuorinen. The Cantor function. Expo. Math., $24(1): 1-37,2006$.

[21] M. J. Edmundo. Structure theorems for o-minimal expansions of groups. Ann. Pure Appl. Logic, 102(1-2):159-181, 2000.

[22] P. Eleftheriou. Small sets in mann pairs. arXiv:1812.07970, 2018.

[23] P. Eleftheriou, A. Hasson, and G. Keren. On definable skolem functions in weakly o-minimal nonvaluational structures. The Journal of Symbolic Logic, 82(4):1482-1495, Dec. 2017.

[24] R. Engelking. Dimension theory. North-Holland Publishing Co., Amsterdam-Oxford-New York; PWN-Polish Scientific Publishers, Warsaw, 1978. Translated from the Polish and revised by the author, North-Holland Mathematical Library, 19.

[25] A. J. Engler and A. Prestel. Valued fields. Springer Monographs in Mathematics. SpringerVerlag, Berlin, 2005.

[26] A. Fornasiero, P. Hieronymi, and C. Miller. A dichotomy for expansions of the real field. Proc. Amer. Math. Soc., 141(2):697-698, 2013.

[27] A. Fornasiero, P. Hieronymi, and E. Walsberg. How to avoid a compact set. Adv. Math., 317:758-785, 2017.

[28] J. M. Fraser and H. Yu. New dimension spectra: finer information on scaling and homogeneity. Adv. Math., 329:273-328, 2018.

[29] H. Friedman, K. Kurdyka, C. Miller, and P. Speissegger. Expansions of the real field by open sets: definability versus interpretability. J. Symbolic Logic, 75(4):1311-1325, 2010.

[30] H. Friedman and C. Miller. Expansions of o-minimal structures by sparse sets. Fund. Math., 167(1):55-64, 2001.

[31] I. García, K. Hare, and F. Mendivil. Assouad dimensions of complementary sets. Proc. Roy. Soc. Edinburgh Sect. A, 148(3):517-540, 2018.

[32] J. Goodrick. A monotonicity theorem for DP-minimal densely ordered groups. J. Symbolic Logic, 75(1):221-238, 2010.

[33] A. B. Gorman, P. Hieronymi, and E. Kaplan. Pairs of theories satisfying a mordell-lang condition, 2018.

[34] A. Günaydin and P. Hieronymi. Dependent pairs. J. Symbolic Logic, 76(2):377-390, 2011.

[35] Y. Halevi, A. Hasson, and F. Jahnke. A conjectural classification of strongly dependent fields. Bull. Symb. Log., 25(2):182-195, 2019.

[36] D. Haskell and D. Macpherson. Cell decompositions of C-minimal structures. Ann. Pure Appl. Logic, 66(2):113-162, 1994.

[37] P. Hieronymi. Defining the set of integers in expansions of the real field by a closed discrete set. Proc. Amer. Math. Soc., 138(6):2163-2168, 2010.

[38] P. Hieronymi. Expansions of the ordered additive group of real numbers by two discrete subgroups. J. Symbolic Logic, 81(3):1007-1027, 2016.

[39] P. Hieronymi. A tame Cantor set. J. Eur. Math. Soc. (JEMS), 20(9):2063-2104, 2018.

[40] P. Hieronymi and C. Miller. Metric dimensions and tameness in expansions of the real field. Trans. Amer. Math. Soc., accepted.

[41] P. Hieronymi and T. Nell. Distal and non-distal pairs. J. Symb. Log., to appear, arXiv:1511.00130, 2016.

[42] P. Hieronymi, T. Nell, and E. Walsberg. Wild theories with o-minimal open core. Ann. Pure Appl. Logic, 169(2):146-163, 2018.

[43] P. Hieronymi and E. Walsberg. On continuous functions definable in expansions of the ordered real additive group. Preprint, arXiv:1709.03150, 2017.

[44] P. Hieronymi and E. Walsberg. Interpreting the monadic second order theory of one successor in expansions of the real line. Israel J. Math., 224(1):39-55, 2018. 
[45] P. Hieronymi and E. Walsberg. Fractals and the monadic second order theory of one successor. Preprint, arXiv:1901.03273, 2019.

[46] E. Hrushovski, Y. Peterzil, and A. Pillay. On central extensions and definably compact groups in o-minimal structures. J. Algebra, 327:71-106, 2011.

[47] E. Hrushovski and A. Pillay. On NIP and invariant measures. J. Eur. Math. Soc. (JEMS), 13(4):1005-1061, 2011.

[48] U. Hrushovski and A. Pillay. Weakly normal groups. In Logic colloquium '85 (Orsay, 1985), volume 122 of Stud. Logic Found. Math., pages 233-244. North-Holland, Amsterdam, 1987.

[49] W. Johnson. The canonical topology on dp-minimal fields. J. Math. Log., 18(2):1850007, 23, 2018.

[50] I. Kaplan, A. Onshuus, and A. Usvyatsov. Additivity of the dp-rank. Trans. Amer. Math. Soc., 365(11):5783-5804, 2013.

[51] T. Kawakami, K. Takeuchi, H. Tanaka, and A. Tsuboi. Locally o-minimal structures. J. Math. Soc. Japan, 64(3):783-797, 2012.

[52] G. Keren. Definable compactness in weakly o-minimal structures. Master's thesis, Ben Gurion University of the Negev, 2014.

[53] T. Kuijpers and E. Leenknegt. Differentiation in $P$-minimal structures and a $p$-adic local monotonicity theorem. J. Symb. Log., 79(4):1133-1147, 2014.

[54] M. C. Laskowski and C. Steinhorn. On o-minimal expansions of Archimedean ordered groups. J. Symbolic Logic, 60(3):817-831, 1995.

[55] O. Le Gal and J.-P. Rolin. An o-minimal structure which does not admit $C^{\infty}$ cellular decomposition. Ann. Inst. Fourier (Grenoble), 59(2):543-562, 2009.

[56] J. Luukkainen. Assouad dimension: antifractal metrization, porous sets, and homogeneous measures. J. Korean Math. Soc., 35(1):23-76, 1998.

[57] D. Macpherson, D. Marker, and C. Steinhorn. Weakly o-minimal structures and real closed fields. Trans. Amer. Math. Soc., 352(12):5435-5483, 2000.

[58] N. Mariaule. Model theory of the field of $p$-adic numbers expanded by a multiplicative subgroup. arXiv:1803.10564, 2018.

[59] D. Marker, Y. Peterzil, and A. Pillay. Additive reducts of real closed fields. J. Symbolic Logic, 57(1):109-117, 1992.

[60] D. Marker and C. I. Steinhorn. Definable types in o-minimal theories. J. Symbolic Logic, 59(1):185-198, 1994.

[61] C. Michaux and R. Villemaire. Presburger arithmetic and recognizability of sets of natural numbers by automata: new proofs of Cobham's and Semenov's theorems. Ann. Pure Appl. Logic, 77(3):251-277, 1996.

[62] C. Miller. Expansions of dense linear orders with the intermediate value property. J. Symbolic Logic, 66(4):1783-1790, 2001.

[63] C. Miller. Tameness in expansions of the real field. In Logic Colloquium '01, volume 20 of Lect. Notes Log., pages 281-316. Assoc. Symbol. Logic, Urbana, IL, 2005.

[64] C. Miller and P. Speissegger. Corrections to "expansions of the real field by canonical product", 2020, available at https://people.math.osu.edu/miller.1987/prodfix.pdf.

[65] C. Miller and P. Speissegger. Expansions of the real line by open sets: o-minimality and open cores. Fund. Math., 162(3):193-208, 1999.

[66] C. Miller and J. Tyne. Expansions of o-minimal structures by iteration sequences. Notre Dame J. Formal Logic, 47(1):93-99, 2006.

[67] A. Nesin and A. Pillay. Some model theory of compact Lie groups. Trans. Amer. Math. Soc., 326(1):453-463, 1991.

[68] A. Onshuus and A. Usvyatsov. On dp-minimality, strong dependence and weight. J. Symbolic Logic, 76(3):737-758, 2011.

[69] A. Onshuus and M. Vicaria. Definable groups in models of presburger arthimetic and $g^{00}$. preprint, 2017.

[70] Y. Peterzil and S. Starchenko. A trichotomy theorem for o-minimal structures. Proc. London Math. Soc. (3), 77(3):481-523, 1998.

[71] A. Pillay. First order topological structures and theories. J. Symbolic Logic, 52(3):763-778, 1987.

[72] S. Shelah. Dependent first order theories, continued. Israel J. Math., 173:1-60, 2009.

[73] S. Shelah and P. Simon. Adding linear orders. J. Symbolic Logic, 77(2):717-725, 2012.

[74] P. Simon. On dp-minimal ordered structures. J. Symbolic Logic, 76(2):448-460, 2011. 
[75] P. Simon. A guide to NIP theories, volume 44 of Lecture Notes in Logic. Cambridge University Press, 2015.

[76] P. Simon. VC-sets and generic compact domination. Israel J. Math., 218(1):27-41, 2017.

[77] P. Simon and E. Walsberg. Dp and other minimalities. Preprint, arXiv:1909.05399, 2019.

[78] P. Simon and E. Walsberg. Tame topology over dp-minimal structures. Notre Dame J. Form. Log., 60(1):61-76, 2019.

[79] A. Thamrongthanyalak. Dimensional coincidence does not imply measure theoretic tameness. Fundamenta Mathematicae, to appear, 2017.

[80] C. Toffalori and K. Vozoris. Notes on local o-minimality. MLQ Math. Log. Q., 55(6):617-632, 2009.

[81] M. C. Tran and E. Walsberg. A family of dp-minimal expansions of $(\mathbb{Z} ;+), 2017$.

[82] M. A. Tychonievich. Tameness results for expansions of the real field by groups. ProQuest LLC, Ann Arbor, MI, 2013. Thesis (Ph.D.)-The Ohio State University.

[83] L. van den Dries. The field of reals with a predicate for the powers of two. Manuscripta Math., 54(1-2):187-195, 1985.

[84] L. van den Dries. A generalization of the Tarski-Seidenberg theorem, and some nondefinability results. Bull. Amer. Math. Soc. (N.S.), 15(2):189-193, 1986.

[85] L. van den Dries. Tame topology and o-minimal structures, volume 248 of London Mathematical Society Lecture Note Series. Cambridge University Press, Cambridge, 1998.

[86] L. van den Dries and A. Günaydı n. The fields of real and complex numbers with a small multiplicative group. Proc. London Math. Soc. (3), 93(1):43-81, 2006.

[87] L. van den Dries and A. H. Lewenberg. T-convexity and tame extensions. J. Symbolic Logic, 60(1):74-102, 1995.

[88] E. Walsberg. Dp-minimal expansions of $(\mathbb{Z},+)$ via dense pairs via mordell-lang. forthcoming.

[89] V. Weispfenning. Mixed real-integer linear quantifier elimination. In Proceedings of the 1999 International Symposium on Symbolic and Algebraic Computation (Vancouver, BC), pages 129-136 (electronic). ACM, New York, 1999.

[90] R. Wencel. Weakly o-minimal nonvaluational structures. Ann. Pure Appl. Logic, 154(3):139$162,2008$.

[91] R. Wencel. On the strong cell decomposition property for weakly o-minimal structures. $M L Q$ Math. Log. Q., 59(6):452-470, 2013.

Department of Mathematics, Statistics, and Computer Science, Department of Mathematics, University of California, Irvine, 340 Rowland Hall (Bldg.\# 400), Irvine, CA 92697-3875

E-mail address: ewalsber@uci.edu

URL: http://www.math.illinois.edu/ ${ }^{\sim}$ erikw 